\title{
BUBBLE ENTRAINMENT AND LIQUID-BUBBLE INTERACTION UNDER UNSTEADY BREAKING WAVES
}

\author{
by \\ Morteza Derakhti
}

A thesis submitted to the Faculty of the University of Delaware in partial fulfillment of the requirements for the degree of Master of Civil Engineering

Fall 2013

(C) 2013 Morteza Derakhti

All Rights Reserved 


\title{
BUBBLE ENTRAINMENT AND LIQUID-BUBBLE INTERACTION UNDER UNSTEADY BREAKING WAVES
}

\author{
by \\ Morteza Derakhti
}

Approved:

James T. Kirby, Ph.D.

Professor in charge of thesis on behalf of the Advisory Committee

Approved:

Harry W. Shenton III, Ph.D.

Chair of the Department of Civil and Environmental Engineering

Approved:

Babatunde Ogunnaike, Ph.D.

Dean of the College of Engineering

Approved:

James G. Richards, Ph.D.

Vice Provost for Graduate and Professional Education 


\section{ACKNOWLEDGMENTS}

Foremost, I would like to express my sincere gratitude to my advisor Prof. James Kirby for his patience, motivation, enthusiasm, and immense knowledge. His guidance helped me in all the time of research and writing of this thesis. I would also like to thank the Civil and Environmental Engineering Department at the University of Delaware for their support.

I gratefully acknowledge financial support from the office of Naval Research, Littoral Geosciences and Optics Program (grants N00014-10-1-0088 and N00014-13-10124). This research was supported in part through the use of computational resources provided by Information Technologies at the University of Delaware. 


\section{TABLE OF CONTENTS}

LIST OF TABLES . . . . . . . . . . . . . . . . . . . . . vi

LIST OF FIGURES . . . . . . . . . . . . . . . . . . . vii

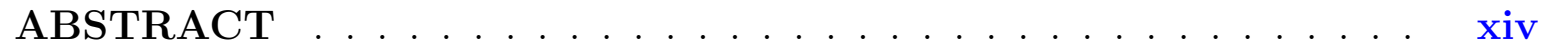

\section{Chapter}

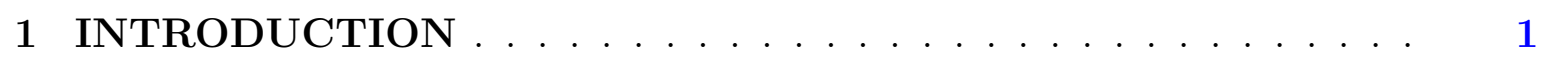

2 MATHEMATICAL FORMULATION AND NUMERICAL

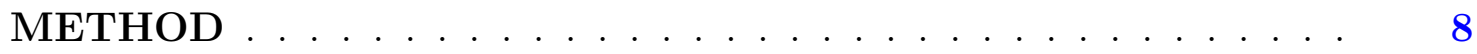

2.1 The Filtered Two-Fluid Equations . . . . . . . . . . . . . . . . . . . 8

2.2 Interfacial Momentum Exchange . . . . . . . . . . . . . . . . . . . . 11

2.3 Bubble Entrainment Model . . . . . . . . . . . . . . . . . . . . . . 12

2.4 Subgrid-Scale Model . . . . . . . . . . . . . . . . . . . . . . 13

2.5 Free Surface Tracking . . . . . . . . . . . . . . . . . . . . 16

2.6 Boundary Conditions . . . . . . . . . . . . . . . . . . . . . . . . . . . . . . . . . . . . .

2.7 Numerical Method . . . . . . . . . . . . . . . . . . . . . 17

2.8 Reynolds Decomposition Of The Resolved Fields . . . . . . . . . . . . 18

3 NUMERICAL SIMULATIONS . . . . . . . . . . . . . . . 20

3.1 Grid Size Selection For 3D Simulations . . . . . . . . . . . . . 21

3.1.1 Free surface evolution . . . . . . . . . . . . . . . 21

3.1.2 Resolved turbulent fluctuations . . . . . . . . . . . 22

3.2 Model Set-Up . . . . . . . . . . . . . . . . . 25

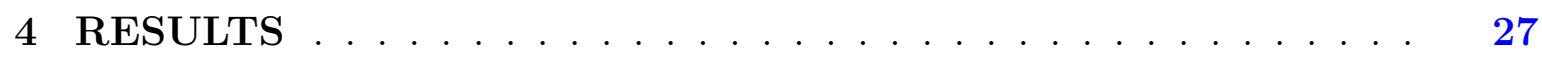

4.1 Bubble Entrainment And Transport . . . . . . . . . . . . . 27

4.1.1 3D free surface evolution and entrainment mechanisms . . . . 27 
4.1 .2 Void fraction distributions . . . . . . . . . . . . . . . 32

4.1.3 Integral properties of the bubble plume . . . . . . . . . 34

4.2 Nonlinear Interaction Of Wave Packet Components . . . . . . . . . 38

4.3 Spanwise Averaged Velocity And Vorticity . . . . . . . . . . . . . 42

4.4 Turbulent Velocity And Vorticity . . . . . . . . . . . . . . . 47

4.5 TKE And Enstrophy . . . . . . . . . . . . . . . . . . . 52

4.6 Reynolds Stress . . . . . . . . . . . . . . . . . . . . 56

4.7 Energy Dissipation . . . . . . . . . . . . . . . 56

4.7.1 Shear- and bubble-induced dissipation . . . . . . . . . 58

4.7.2 Time dependent breaking parameter, $b \ldots \ldots \ldots$

4.8 Liquid-Bubble Momentum Exchange And Production By The Dispersed Bubbles . . . . . . . . . . . . . . . 63

4.9 TKE Transport In The Breaking Region . . . . . . . . . . 67

4.9.1 The two phase vs single phase TKE transport equation . . . 72

4.9.2 Turbulence modulation by the dispersed bubbles . . . . . . . 72

4.10 Velocity Spectra . . . . . . . . . . . . . . . . . . . 73

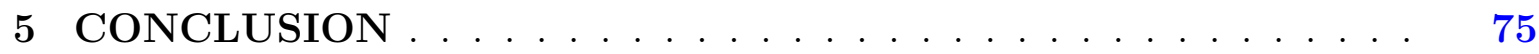

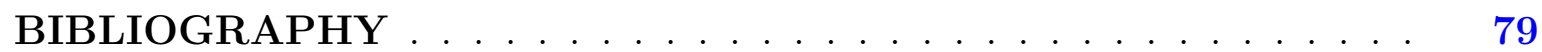

\section{Appendix}

A VOID VS ACTUAL AIR ................ 84

B EXACT TRASPORT EQUATIONS FOR TURBULENT BUBBLY

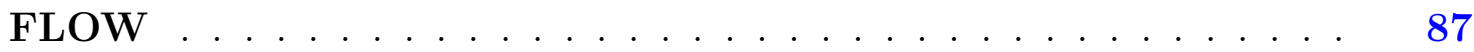

B.1 Transport Equation For Averaged Resolved Kinetic Energy . . . . . . 87

B.2 Transport Equation For Resolved Turbulent Kinetic Energy . . . . . 88

B.3 Transport Equation For Kinetic Energy Of Organized Flow . . . . . . 91 


\section{LIST OF TABLES}

3.1 Input parameters for the simulated cases . . . . . . . . . . . . 21

3.2 Input parameters for additional experiments which are similar with the simulated cases . . . . . . . . . . . . . . 21

$3.3 \quad$ Numerical set-up for the 3D LES simulated cases . . . . . . . . . 26

3.4 Model input parameters for the 3D LES simulations . . . . . . . . . 26

4.1 Total dissipation in the breaking region. LM approximated the total dissipation by estimating the total energy flux difference between upstream and downstream of the breaking region. . . . . . . . . 


\section{LIST OF FIGURES}

3.1 Grid dependence study of the free surface evolution (contours of $f=0.5$ ) during active breaking for P1. a) Snapshots of the overturning process and splash-up and $b$ ) Time history of the surface elevation at $x^{*}=0.285$. The different $2 \mathrm{D}$ mesh resolutions are $(\Delta x \times \Delta z),-15 \times 4.6 \mathrm{~mm},-22.7 \times 7 \mathrm{~mm},-\cdot-33.3 \times$ $10.5 \mathrm{~mm},---50 \times 16 \mathrm{~mm}$. Circles are the measurement and adopted from LM figure $1(\mathrm{a}) \ldots \ldots \ldots \ldots$

3.2 Grid dependence study of the resolved turbulent fluctuations for P1. a) total spanwise averaged resolved TKE, $\overline{\tilde{k}}$ ratio to the total spanwise averaged TKE and $b$ ) normalized total spanwise averaged SGS TKE, $\check{\bar{k}}_{s g s .}-\Delta x=23.1 \mathrm{~mm}, \Delta y=7 \mathrm{~mm}, \Delta z=7 \mathrm{~mm}$, $\triangle x=34 \mathrm{~mm}, \Delta y=10.5 \mathrm{~mm}, \Delta z=10.5 \mathrm{~mm}$, $-\cdot-\Delta x=50 \mathrm{~mm}, \Delta y=16 \mathrm{~mm}, \Delta z=16 \mathrm{~mm}$. The reference value for $\check{\bar{k}}_{s g s}$ is $L_{c}^{2} C_{c}^{2} \ldots \ldots \ldots \ldots \ldots \ldots \ldots \ldots \ldots$

4.1 Snapshots of the free surface (isosurface of $f=0.5$ ) evolution for P1

4.2 Snapshots of 3D bubble plume (isosurface of $\alpha^{b}=0.05 \%$ ) evolution in the breaking region for P1. (left) side view of the $3 \mathrm{~d}$ results and (right) top view of the $3 \mathrm{~d}$ results. . . . . . . . . . . . . . .

4.3 Snapshots of 3D bubble plume (isosurface of $\alpha^{b}=0.05 \%$ ) evolution in the breaking region for SP1. (left) side view of the 3d results and (right) top view of the $3 \mathrm{~d}$ results. . . . . . . . . . . .

4.4 Time-dependent contour plots of the spanwise averaged void fraction distributions $\left(\bar{\alpha}^{b \%} \%\right)$ in the breaking region for P1 . . . . . . . . 31

4.5 Time-dependent contour plots of the spanwise averaged void fraction distributions $\left(\bar{\alpha}^{b \%} \%\right)$ in the breaking region for SP1. . . . . . . . 32 
4.6 Time averaged vertically integrated spanwise averaged volume of the dispersed bubbles, $\bar{V}_{b}$ and --- the cavities captured by the VOF model, $\overline{\bar{V}}_{c}$. (left) P1; (middle) P2 and (right) SP1. . . . . . .

4.7 Integral properties of the bubble plume for P1. a) Normalized volume of the entrained air, $\left.-V^{b} / V_{0},---\left(V^{b}+V^{c}\right) / V_{0} ; b\right)$ normalized bubble plume area $-A^{b} / V_{0}$, and c) averaged volume fraction (\%), $-\alpha_{\text {ave }}^{b},---\left(V^{b}+V^{c}\right) / A^{b}$. Total volume of the cavities captured by the VOF model is $V^{c}=\int_{A}(1-\bar{f}) d A$. $\cdots \cdots$ Experimental fitted curves from LM. Circles are the measurements of the corresponding

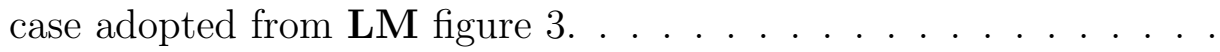

4.8 Integral properties of the plume for SP1. Definitions are the same as

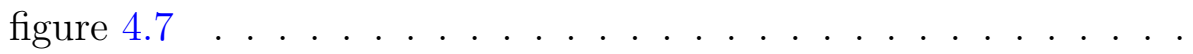

4.9 Normalized centroid positions of the bubble plume for — P1, -- SP1. . . . . . . . . . . . . . . .

4.10 Free surface time series near the breaking point for P3. Circles are the measurements of the corresponding case adopted from RM figure $7(\mathrm{a}) \ldots \ldots \ldots \ldots \ldots \ldots$

4.11 Power density spectra of the wave packet for (left) incipient breaking case, $S=0.257$ and (right) P3. The numbers on the graphs are the values of $k_{c}\left(x-x_{b}\right)$. The dash line corresponds to the spectra at $k_{c}\left(x-x_{b}\right)=-15$ for each column. Vertical dotted lines show the frequency band boundaries of the input wave packet. . . . . . . .

4.12 Modulus of the wavelet transform of (left) the linear packet, $S=0.05$; (middle) incipient breaking and (right) P3. The numbers on the graphs are the values of $k_{c}\left(x-x_{b}\right)$. Horizontal dotted lines show the frequency band boundaries of the input wave packet. — shows the arrival time of the different frequencies based on the linear theory group velocity. ...................

4.13 Phase angle, $\Theta_{B_{\bar{\eta}}}$, of the spanwise averaged free surface bispectrum $\left.B_{\bar{\eta}}\left(s_{1}, s_{1}, t\right)\right)$, for $\cdots \cdots$ linear packet, $S=0.05$; -- incipient breaking and — P3. The numbers on the graphs are the values of $f_{1} / f_{c}$, where $f_{1}=1 / c s_{1}$ and $c=4 \pi /\left(\omega_{0}+\sqrt{2+\omega_{0}^{2}}\right)$ is a constant. . 
4.14 a) Spatial distribution of the normalized mean current, $\mathbf{u}_{\mathbf{c}}^{*} ; b$ ) normalized horizontal averaged mean current in the streamwise direction, $\widehat{u_{c}^{*}}$ and $c$ ) normalized accumulative horizontal averaged mass flux, $\widehat{M^{*}}$, in the breaking region for P3. Colors show $\sqrt{u_{c}^{* 2}+v_{c}^{* 2} \ldots \ldots \ldots \ldots \ldots \ldots \ldots \ldots \ldots \ldots \ldots \ldots \ldots \ldots}$

4.15 Modulus of the wavelet transform of $\overline{u^{*}}-u_{c}^{*}$ for P3. (left) $x^{*}=0.3$; (middle) $x^{*}=0.6$ and (right) $x^{*}=1.05$. The elevation of corresponding signal location in each row is the same and is shown at the left column. Dotted lines indicate the frequency band of the input packet. Dashed line shows the cut-off frequency for low-pass filtering. Regions below the solid lines on either end indicate the ?cone of influence,? where edge effects become important. . . . . . . . . .

4.16 (left) Normalized low-pass filtered spanwise averaged velocity field, $\left(\overline{u^{*}}, \overline{v^{*}}\right)$ and (right) normalized vorticity of the organized flow, $\overline{\omega_{y}^{*}}$, for P3. The arrays are normalized in each figure and the color shows the

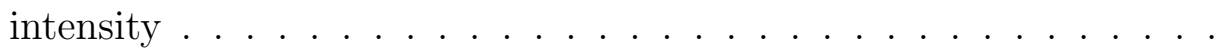

4.17 Normalized low-pass filtered spanwise averaged and r.m.s. velocities for P3 at $(a-b) x^{*}=0.15$ and $(c-d) x^{*}=0.60$ in different elevations. Circles are the measurements of the corresponding case adopted from RM figure 42 and figure 46. . . . . . . . . . . . . .

4.18 Snapshots of turbulent r.m.s. of $a$ ) velocities and b) vorticity components for $\mathrm{P} 1 . \ldots \ldots \ldots \ldots$. . . . . . . . . . .

4.19 Horizontally averaged turbulent velocity and vorticity variance for P1. $(a-d)-\widehat{\overline{u^{\prime 2}}} ;-\cdot-\widehat{\widehat{w^{\prime 2}}} ;---\widehat{\overline{v^{\prime 2}}}$ and $(e-h)-\widehat{\widehat{\omega_{x}^{\prime 2}}}$; $-\cdot-\widehat{\widehat{\omega_{z}^{\prime 2}}} ;--\widehat{\widehat{\omega_{y}^{\prime 2}}} \cdot(a, e) t^{*}=0.5 ;(b, f) t^{*}=1.0 ;(c, g) t^{*}=2.0$ and $(d, h) t^{*}=4.0 \ldots \ldots \ldots \ldots \ldots \ldots$

4.20 Horizontally averaged turbulent velocity and vorticity variance for SP1. $(a-d)-\widehat{\widehat{u^{\prime 2}}} ;-\cdot-\widehat{\widehat{w^{\prime 2}}} ;---\widehat{\widehat{v^{\prime 2}}}$ and $(e-h)-\widehat{\widehat{\omega_{x}^{\prime 2}}}$; $-\cdot \widehat{\widehat{\omega_{z}^{\prime 2}}} ;--\widehat{\widehat{\omega_{y}^{\prime 2}}} \cdot(a, e) t^{*}=0.5 ;(b, f) t^{*}=1.0 ;(c, g) t^{*}=2.0$ and $(d, h) t^{*}=4.0 \ldots \ldots \ldots \ldots \ldots \ldots$

4.21 Ratios of the different turbulent fluctuation components to total

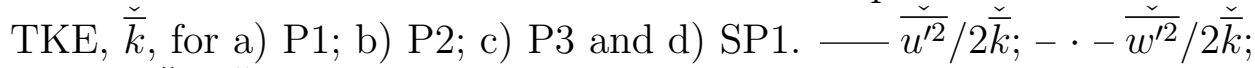
$\ldots \ldots \overline{v^{\prime 2}} / 2 \check{k} \ldots \ldots \ldots \ldots \ldots \ldots \ldots$ 
4.22 Ratios of the different turbulent vorticity components to mean square turbulent vorticity, $\widetilde{\omega^{\prime 2}}$, for a) P1; b) P2; c) P3 and d) SP1.

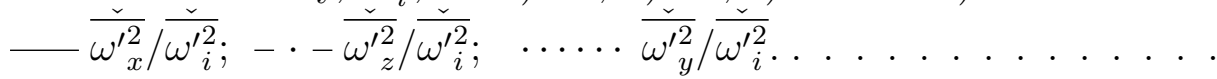

4.23 Snapshots of the normalized spanwise averaged TKE, $\bar{k}$, for (left) P1; (middle) SP1 and (right) P3. The reference value is $C_{c}^{2}$. . . . .

4.24 Snapshots of the normalized spanwise averaged enstrophy, $\bar{\Omega}$, for (left) P1; (middle) SP1 and (right) P3. The reference value is $T_{c}^{-2}$.

4.25 a) Total TKE, $\check{\bar{k}}$ for P3; b) total TKE, $\check{\bar{k}}$ and $c$ ) total enstrophy $\check{\bar{\Omega}}$ for $-\mathrm{P} 1,-\cdots-\mathrm{P} 2$ and $---\mathrm{SP} 1$. Dotted lines indicate $\left(t^{*}\right)^{n}$ where $n$ is the number on the lines. Circles are $\sum_{i=1}^{7} \sum_{j=1}^{7} \frac{1}{2}\left(<u^{\prime 2}(i, j)>+2<w^{\prime 2}(i, j)>\right) A(i, j)$ adopted from RM figure 54. The reference values for TKE and enstrophy are $L_{c}^{2} C_{c}^{2}$ and $L_{c}^{2} T_{c}^{-2}$, respectively. . . . . . . . . . . . . . . .

4.26 The two different estimates of three-dimensional TKE for a) P1; b) P2; c) P3 and d) SP1. _ $\check{\bar{k}}^{1} / \check{\bar{k}} ; \ldots \ldots \check{\bar{k}}^{1} / \check{\bar{k}} \ldots \ldots . . . .$.

4.27 Spanwise averaged Reynold stress in longitudinal plane, $\overline{u^{\prime} w^{\prime}} / C_{c}^{2}$ for (left) P1 and (right) SP1. . . . . . . . . . . . . .

4.28 Snapshots of the spanwise averaged SGS dissipation rate, $\overline{\varepsilon_{\text {sgs }}}\left[m^{2} s^{-3}\right]$ for P1, from the simulation (left) with and (right) without the inclusion of the dispersed bubbles. . . . . . . . . . . . . . . .

4.29 Dissipation rate per unit length of crest due to the different mechanisms for $a) \mathrm{P} 1$; b) P2; c) SP1 and $d) \mathrm{S} 1$. _ shear-induced $\check{\varepsilon_{S I}} / L_{c}^{2}, \ldots \ldots$ bubble-induced $\check{\check{\varepsilon_{B I}}} / L_{c}^{2}$, and $-\cdot-$ viscous dissipation $\check{\varepsilon_{r}} / L_{c}^{2}$. Dashed lines indicate $\left(t^{*}\right)^{n}$ where $n$ is the number on the lines. . . . . . . . . . . . . . . . . . . .

4.30 Total breaking-induced dissipation per unit length of crest $(\mathrm{J} / \mathrm{m})$ for a) $\mathrm{P} 1 ; b) \mathrm{P} 2 ; c) \mathrm{SP} 1$ and $d) \mathrm{S} 1 .-\hat{\epsilon}_{\text {total }}=\hat{\epsilon}_{r}+\hat{\epsilon}_{s g s}, \quad---\hat{\epsilon}_{s g s}^{S I}$, $-\cdot-\hat{\epsilon}_{s g s}^{B I}, \cdots \cdots \hat{\epsilon}_{r},-\hat{\epsilon}_{\text {total }}^{n b}$ is the total dissipation from the simulations without the inclusion of the dispersed bubbles. . . . . .

4.31 Different breaking parameters $a) b ; b) \beta$ and $c) \overline{\bar{\beta}}$ for $-\mathrm{P} 1$; - - $\mathrm{P} 2 ;--\mathrm{SP}_{2} ; \ldots . \mathrm{S} 1 ;-\mathrm{P} 3$ and $---\mathrm{S} 2 . \ldots . .$. 
4.32 Total spanwise averaged and r.m.s. of the interfacial forces exerted on the water column by the bubbles, $(a, b, c) \check{F}_{i, m}^{\text {type }}$ and $(d, e, f) \check{F}_{i, r m s}^{\text {type }}$ for P1. $(a, d)$ streamwise direction; $(b, e)$ vertical direction; $(c, f)$ spanwise direction. — drag force, $\cdots \cdots$ virtual mass force, --- lift force. The reference value is $\rho L_{c}^{2} C_{c} T_{c}^{-1}$. . . . . .

$4.33(a, b, c) \check{F}_{i, m}^{\text {type }}$ and $(d, e, f) \check{F}_{i, r m s}^{\text {type }}$ for SP1. The definitions are the same

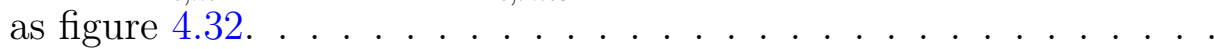

4.34 The rate of work done on the organized and turbulent flow by the dispersed bubbles, $(a, b, c) \check{B}_{i, t y p e}^{m}$ and $(d, e, f) \check{B}_{i, t y p e}^{k}$ for P1. $(a, d)$ streamwise direction; $(b, e)$ vertical direction; $(c, f)$ spanwise direction. — drag force, $\cdots \cdots$ virtual mass force, --- lift force. The reference value is $\rho L_{c}^{2} C_{c}^{2} T_{c}^{-1} \ldots \ldots \ldots \ldots$. . . . . . .

$4.35(a, b, c) \check{B}_{i, t y p e}^{m}$ and $(d, e, f) \check{B}_{i, t y p e}^{k}$ for SP1. The definitions are the

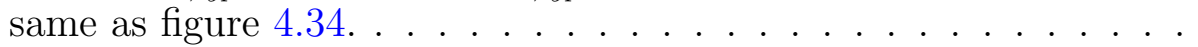

4.36 Total work done by _ drag, $\ldots .$. virtual mass, and - - lift force, on $(a, b)$ turbulent motions and $(c, d)$ organized flow; for $(a, c)$ $\mathrm{P} 1$ and $(b, d) \mathrm{SP} 1$. The reference value is $\rho L_{c}^{2} C_{c}^{2} \ldots \ldots . . .$.

$4.37(a, b)$ Total rate of work done by the dispersed bubbles on — TKE and - - the organized flow for $a$ ) $\mathrm{P} 1 ; b) \mathrm{SP} 1$. The reference value is $\rho L_{c}^{2} C_{c}^{2} T_{c}^{-} 1$. (c,d) Total work done by the dispersed bubbles on $\mathrm{TKE}$ and --- the organized flow for $c$ ) P1; $d$ )SP1. The reference value is $\rho L_{c}^{2} C_{c}^{2} \ldots \ldots \ldots \ldots \ldots \ldots$

4.38 Schematic of the different production and dissipation mechanisms of two phase bubbly flow TKE. . . . . . . . . . . . . . . .

4.39 Total production rate by mean shear in the breaking region based on the two phase $-\check{P}^{k}+\check{P}_{e x 1}^{k}+\check{P}_{e x 2}^{k}$ and the single phase transport equation $\cdots \ldots \check{P}^{S P}$, for $\left.\left.\left.a\right) \mathrm{P} 1 ; b\right) \mathrm{P} 2 ; c\right) \mathrm{SP} 1$ and $\left.d\right) \mathrm{S} 1$. The reference value is $\rho L_{c}^{2} C_{c}^{2} T_{c}^{-1} \ldots \ldots \ldots \ldots \ldots$

4.40 Total SGS dissipation rate in the breaking region based on the two phase $-\check{\epsilon}_{s g s}^{k}+\check{\epsilon}_{s g s, e x 1}^{k}+\check{\epsilon}_{s g s, e x 2}^{k}$ and the single phase transport equation $\cdots \cdots \check{\epsilon}_{s g s}^{S P}$ for $\left.\left.\left.a\right) \mathrm{P} 1 ; b\right) \mathrm{P} 2 ; c\right) \mathrm{SP} 1$ and $\left.d\right) \mathrm{S} 1$. The reference value is $\rho L_{c}^{2} C_{c}^{2} T_{c}^{-1}$. 
4.41 Different terms in the total production rate by mean shear $-\check{P}^{k}$; $---\check{P}_{e x 1}^{k}$ and $\ldots . . \check{P}_{e x 2}^{k}$ for $\left.\left.\left.a\right) \mathrm{P} 1 ; b\right) \mathrm{P} 2 ; c\right) \mathrm{SP} 1$ and $\left.d\right) \mathrm{S} 1$. The reference value is $\rho L_{c}^{2} C_{c}^{2} T_{c}^{-1} \ldots \ldots \ldots \ldots . \ldots . \ldots$

4.42 Different terms in the total SGS dissipation rate $-\check{\epsilon}_{s g s}^{k} ; \quad---$ $\check{\epsilon}_{s g s, e x 1}^{k}$ and $\cdots \cdots \check{\epsilon}_{s g s, e x 2}^{k}$ for $\left.\left.a\right) \mathrm{P} 1 ; b\right) \mathrm{P} 2$; c) SP1 and $\left.d\right) \mathrm{S} 1$. The reference value is $\rho L_{c}^{2} C_{c}^{2} T_{c}^{-1} \ldots \ldots \ldots \ldots \ldots$

4.43 The two phase total production rate by mean shear $\check{P}_{\text {total }}^{k}=\check{P}^{k}+\check{P}_{e x 1}^{k}+\check{P}_{e x 2}^{k}$ from - simulation with dispersed bubbles and --- simulation without inclusion of the dispersed bubbles for a) $\mathrm{P} 1 ; b) \mathrm{P} 2 ; c) \mathrm{SP} 1$ and $d) \mathrm{S} 1$. The reference value is $\rho L_{c}^{2} C_{c}^{2} T_{c}^{-1}$.

4.44 The two phase total SGS dissipation rate by mean shear $\check{\epsilon}_{\text {total }}^{k}=\check{\epsilon}^{k}+\check{\epsilon}_{e x 1}^{k}+\check{\epsilon}_{e x 2}^{k}$ from $\longrightarrow$ simulation with dispersed bubbles and --- simulation without inclusion of the dispersed bubbles for a) $\mathrm{P} 1 ; b) \mathrm{P} 2 ; c) \mathrm{SP} 1$ and $d) \mathrm{S} 1$. The reference value is $\rho L_{c}^{2} C_{c}^{2} T_{c}^{-1}$.

4.45 The total production by buoyancy $\check{E}_{p}^{k}$, for a) P1; b) P2; c) SP1 and $d$ ) $\mathrm{S} 1$. The reference value is $\rho L_{c}^{2} C_{c}^{2} T_{c}^{-1} \ldots \ldots \ldots \ldots$

4.46 Normalized total resolved TKE, $\check{k}$, in the breaking region, — simulation with dispersed bubbles and - - - simulation without inclusion of the dispersed bubbles for $a$ ) $\mathrm{P} 1$; b) P2; c) SP1 and $d$ )S1. The reference value is $L_{c}^{2} C_{c}^{2}(S-S 0) S_{0}^{4} \ldots \ldots$. . . . . . . .

4.47 Spanwise averaged energy spectra at different elevations for P3 at $t^{*}=4$. (left) $z^{*}=-0.02$ and (right) $z^{*}=-0.1 .-E_{11} ;---E_{31}$ and $\ldots \ldots E_{21} \ldots \ldots \ldots \ldots$

4.48 Energy spectra at $t^{*}=1, z^{*}=-0.05$ and different $x$ locations for P1. a) out side the turbulent region, $x^{*}=0.2$ and $b$ ) inside the turbulent region, $x^{*}=0.45$. $-E_{12} ;---E_{32}$ and $\ldots \ldots E_{22} . \ldots$

A.1 Snapshots of the time sequences of the overturning process and splash-up for P5. Free surface profiles (contours of $\alpha^{l}=0.5$ ) are shown for different mesh resolutions $(\Delta x \times \Delta z),-10 \times$ $2.5 \mathrm{~mm},-20 \times 5 \mathrm{~mm},---30 \times 10 \mathrm{~mm}$. Circles shows the measurement (adopted from BCh figure 3). . . . . . . . . . . 
A.2 Cavity deformation in the quiescent water (Iso-surface of $\alpha=0.5$ ); (first row) water/void with $23 \times 7 \times 7 \mathrm{~mm}$ resolution; (2nd row) water/air with $23 \times 7 \times 7 \mathrm{~mm}$ resolution; (3rd row) water/air with $7 \times 7 \times 7 \mathrm{~mm}$ resolution . . . . . . . . . . .

A.3 Centroid position of cavity; - - - water/void with $23 \times 7 \times 7 \mathrm{~mm}$ resolution; - - water/air with $23 \times 7 \times 7 \mathrm{~mm}$ resolution; water/air with $7 \times 7 \times 7 \mathrm{~mm}$ resolution; $\cdots \cdots$ shows the free surface location . . . . . . . . . . . . . . 


\begin{abstract}
Wave breaking is a highly dissipative process, and also a source of turbulence in the ocean surface layer. It entrains a large volume of air in bubbles that rapidly evolves into a distribution of bubble sizes which interacts with liquid turbulence and organized motions. The liquid-bubble interaction, especially in the complex two-phase bubbly flow under breaking waves, is still poorly understood. In the present study, we perform a large-eddy simulation (LES) using a Navier-Stokes solver extended to incorporate entrained bubble populations, using an Eulerian-Eulerian formulation for a polydisperse bubble phase. The volume of fluid (VOF) method is used for free surface tracking. We consider an isolated unsteady deep water breaking event imposed by a focussed wave packet. The bubble-induced dissipation and momentum transfer between two phases are considered. The model is shown to predict free surface evolution, mean and turbulent velocities and integral properties of the entrained dispersed bubbles (hereafter bubble plume) fairly well. We investigate bubble plume kinematics and dynamics, turbulence modulation by dispersed bubbles as well as shear- and bubble-induced dissipation, both in spilling and plunging breakers. We find that the total bubble-induced dissipation accounts for more than $50 \%$ of the total dissipation in the breaking region. The averaged dissipation rate per unit length of breaking crest is usually written as $b \rho g^{-1} c^{5}$, where $c$ is the phase speed of the breaking wave. The breaking parameter, $b$, has been poorly constrained by experiments and field measurements. We examine the time dependent evolution of $b$ for both constant-steepness and constant-amplitude wave packets. The scaling law for the averaged breaking parameter is obtained. The exact two-phase transport equation for turbulent kinetic energy (TKE) is compared to the conventional single phase transport equation, and it is found that the former over predicts the total SGS dissipation and turbulence production by
\end{abstract}


mean shear during active breaking. All of the simulations are repeated without the inclusion of dispersed bubble phase, and it is shown that the integrated TKE in the breaking region is damped by the dispersed bubbles about $20 \%$ for the large plunging breaker to $50 \%$ for the spilling breakers. In the plunging breakers, TKE is damped slightly or even enhanced during the initial stage of active breaking. In addition, we examine the nonlinear interaction of different components in a wave packet. Phase locking between spectral components is observed in the breaking region, and explained by calculating the wavelet bispectrum. 


\section{Chapter 1}

\section{INTRODUCTION}

Surface wave breaking is a complex two phase flow phenomenon that has an important role in numerous environmental processes, such as air-sea transfer of gas, momentum and energy, acoustic underwater communications and optical properties of the water column. Wave breaking is a highly dissipative process, limiting the height of surface waves. It is also a source of turbulence which enhances transport and mixing in the ocean surface layer. It entrains a large volume of air in bubbles that rapidly evolves into a distribution of bubble sizes which interacts with liquid turbulence and organized motions. Several experimental studies in a vertical bubble column (e.g. Lance \& Bataille 1991) revealed that the motion of the bubbles relative to the liq-

uid causes velocity fluctuations in the latter and increases the energy of the scales comparable to the bubble diameter. This additional bubble-induced turbulence, commonly called "pseudo-turbulence," is more noticeable during active breaking in which the Kolmogorov length scale is much smaller than the mean diameter of the entrained bubbles. At larger scales, on the other hand, the presence of bubbles can modify liquid turbulence by changing the velocity gradients and the associated change in turbulence production. In addition, work done by the inhomogeneous interfacial forces on the water column can modify larger scale turbulent motions. In shallow water and nearshore regions, this process even becomes more complicated when the bottom effects and sediments alter the flow field.

Bubble plume kinematics and dynamics, and turbulence structures of the bubbly flow under breaking waves are the two main factors that come into play in all of the above mentioned processes (Melville 1996). While the former is well studied experimentally, liquid-bubble interaction, i.e. the effects of dispersed bubbles on organized 
and turbulent motions, is still an open question. Our goal is to have a time-dependent model to resolve both bubble entrainment and transport as well as the large scale of turbulence structures during active breaking. The model must account for turbulence modulation induced by dispersed bubbles and the momentum transfer between two phases. In addition, the large scale free surface features should be captured in order to truly resolve vorticity generation during the jet impact and subsequent splash-up processes.

There are several important reviews on the topic of wave breaking (Banner \& Peregrine 1993, Melville 1996, Duncan 2001). Recently, Kiger \& Duncan (2012) reviewed the mechanisms of air entrainment in plunging jets and breaking waves. Furthermore, Perlin et al. (2012) summarized the different aspects of deep water breaking waves such as geometry, breaking onset and energy dissipation. To summarize the relevant literature on deep water breaking waves, we first review the experimental studies of bubble void fraction as well as velocity field and turbulence, then we discuss the relevant numerical studies.

Many previous researchers have measured air void fraction in the bubbly flow under breaking waves (Lamarre \& Melville 1991, 1994, Deane \& Stokes 2002, Cox \& Shin 2003, Blenkinsopp \& Chaplin 2007, Rojas \& Loewen 2010). Using a conductivity probe, (Lamarre \& Melville 1991, hereafter referred to as LM) and Lamarre \& Melville (1994) measured time-dependent void fraction distributions of focused waves with a constant component steepness. They calculated area, volume, mean void fraction and centroids of the bubble plume. It was shown that these integral properties evolved as a simple function of time and scaled fairly well from small two-dimensional (2D) to larger three-dimensional (3D) laboratory breaking waves. The results showed that the degassing rate was very quickly, and less than $5 \%$ of the initial entrained bubbles remained in the water column one period after breaking. They found that the potential energy of the bubble plume can be 30 to $50 \%$ of the total energy dissipated by breaking. Deane \& Stokes (2002) used a high-speed video camera to measure the bubble size distributions under the laboratory scale breaking imposed by the focused wave method. 
They divided the entrainment process into two distinct mechanisms controlling the bubble size distribution. The first is turbulent fragmentation of the entrapped cavity, which is largely responsible for bubbles larger than the Hinze scale, leading to a bubble number density proportional to $r_{b}^{-10 / 3}$, where $r_{b}$ is the bubble radius. The second is jet interaction and drop impact on the wave face, resulting in smaller bubbles with a number density proportional to $r_{b}^{-3 / 2}$. Small bubbles were entrained for a longer time but larger bubbles were created after cavity fragmentation and degassed relatively quickly. Blenkinsopp \& Chaplin (2007) used two optical fibers to measure the timedependent void fraction under breaking waves. Breaking waves were produced by propagating regular waves over a submerged sloping reef structure. They also found that the integral properties of the bubble plume evolved as a simple function of time. They showed that the bubble plume volume grew linearly to a maximum and then decayed exponentially in time, with the ratio of maximum volume of the entrained air to the initial air tube entrapped by the jet lying between 1.3 and 1.6. They estimated the work required to entrain bubbles against buoyancy was about 4 to $9 \%$ of the total dissipation.

(Rapp \& Melville 1990, hereafter referred to as RM) used LDV and measured ensemble averaged mean and turbulent velocities on a regular grid in the breaking region of a focused wave packet. They found energy dissipation by breaking from $10 \%$ to more than $25 \%$ of pre-breaking wave energy for spilling and plunging breakers respectively, with about $90 \%$ of the total dissipation occurring within four wave periods. Total TKE in the breaking region were found to subsequently decay like $\left(t-t_{o b}\right)^{-1}$, where $t_{o b}$ is an observed breaking time. Melville et al. (2002) and (Drazen \& Melville 2009, hereafter referred to as DM) used DPIV and investigated post breaking velocity field and turbulence, with the work by DM having a finer spatial resolution. All the information was only available starting about 3 periods after breaking in which nearly all of the entrained bubbles were degassed and most of the energy was dissipated. DM chose a plunging breaker imposed by a focus wave packet with a constant component steepness. Ensemble-averaged quantities such as mean and turbulent velocity, TKE 
and Reynolds stress were presented. They computed turbulent wavenumber spectra and horizontally averaged terms in the TKE budget up to 27 periods after breaking. In addition, estimates of the dissipation rate were given. Total TKE was seen to depend approximately on $\left(t-t_{o b}\right)^{-0.75}$ while the surface waves are present, and then begins to decay faster for $t^{*}>12$, where $t^{*}$ is given by (3.1) below. In all three experiments, a large coherent vortex structure was seen in the ensemble-averaged velocity field. It is clear this type of breaking differs from quasi-steady breaking since the turbulent region has a finite length, propagating downstream very slowly compared to phase velocity and deepening.

Measurement of a 3D velocity field in a turbulent bubbly flow under breaking waves is very difficult. In addition, there is no way to exclude entrained bubbles under breaking waves in experimental studies. As a result, it is not possible to examine turbulence and organized flow modulations by entrained dispersed bubbles. The available experimental studies which examined bubble-induced turbulence and liquid-bubble interaction are done mostly in a vertical bubble column with a homogeneous swarm of bubbles released at the bottom of a tank. Numerical models, on the other hand, make it possible to study liquid-bubble interactions under breaking waves. In general, we can divide Eulerian-Eulerian numerical models of bubbly flows into discrete and continuous models. In the discrete type models, the interface between an individual bubble and the liquid is resolved. Obviously, the possible bubble diameter which can be resolved is limited to the grid resolution. To account for bubble size distribution under breaking waves, we need to have a very fine grid resolution about two orders of magnitude smaller than typical LES resolution. In the continuous type models, instead, the interface between an individual bubble and the liquid is not resolved, and the interfacial momentum transfers are considered using the closure models. A critical issue in this approach, especially under breaking waves, is accurately introducing air bubbles into a model using a bubble entrainment formulation (Moraga et al. 2008, Shi et al. 2010, Ma et al. 2011).

As summarized by Perlin et al. (2012), most of the numerical simulations for 
deep water breaking waves are limited to the evolution of a periodic unstable wave train with relatively low-Reynolds numbers $\left(\sim 10^{4}\right)$ and short wave lengths $(<0.3 \mathrm{~m})$ (Chen et al. 1999, Song \& Sirviente 2004, Lubin et al. 2006, Iafrati 2009, 2011, Lubin \& Glockner 2013). This artificial way of leading a wave train to breaking has an advantage in that it represents a more compact computational problem. However, it is not possible to do comparisons with experimental data, except in a qualitative sense. In addition, it is well known that, at such a short scale, surface tension significantly affects the breaking process and fragmentation of bubbles. Furthermore, although wave breaking is initially a fairly $2 \mathrm{D}$ event, the entrainment process is highly $3 \mathrm{D}$ even in the case of a small scale plunger where surface tension appears to be playing a strong role (Kiger \& Duncan 2012). Thus, 2D frameworks can not accurately account for bubble transport and vorticity evolution during and after breaking. These issues suggest that the extension of the results to larger scales has to be done rather cautiously. In these discrete numerical studies, the Navier-Stokes equations are solved both in air and water with a relatively fine spatial resolution that can resolve cavity fragmentation to some extent. Although the density and viscosity of the gas is not equal to real air in most of the previous simulations, consideration of the gas phase considerably increased the computational effort. Chen et al. (1999) performed a 2D direct numerical simulation (DNS) of the two-fluid Navier-Stokes equations combined with the VOF method for surface tracing. They chose a density ratio of 0.01 and a viscosity ratio of 0.4 and found that $80 \%$ of the wave energy was dissipated within three wave periods after breaking, and that the temporal evolution of the total mechanical energy had a $\left(t-t_{o b}\right)^{-1}$ dependence. Lubin et al. (2006) did a 3D LES with the real density and viscosity ratio and found $\mathrm{a}\left(t-t_{o b}\right)^{-0.3}$ dependence for the total mechanical energy evolution. Both simulations captured the sequence of splashes but no quantitative estimates of the entrapped air were given. Iafrati (2009) did a 2D DNS of the two-fluid Navier-Stokes equations combined with a level-set method to capture the interface. The real density ratio was chosen, but the viscosity ratio was set to 0.4 . He examined the effects of breaking intensity (with initial steepness over the range $k a=0.2-0.65$ ) 
on the resulting flow. It was concluded that major energy dissipation occurs locally at the region of small bubbles generated by the fragmentation of the air cavity entrapped by the plunging jet. A scaling law for the amount of air entrapped as a function of the initial wave steepness was found. Iafrati (2011) continued his previous work with focus on the early stage of breaking. The different contributions to energy dissipation was estimated for the different initial steepnesses. He found, in the plunging case, that a fraction between 10 to $35 \%$ of the energy dissipated during breaking was spent in entraining the air cavity against the action of buoyancy force, and most of it was dissipated by viscous effects when the cavity collapsed.

The first attempt to use a continuous type model for studying bubbly flow under breaking waves, to the knowledge of the authors, was done by Shi et al. (2010). They used a 2D VOF based mixture model, with a $k-\varepsilon$ turbulence closure, to study air bubble evolution in an isolated unsteady breaking wave in a laboratory scale event. They used an air bubble entrainment formula which connected shear production at the air-water interface and the bubble number density with the bubble size distributions suggested by Deane \& Stokes (2002). The bubble velocities were calculated directly by adding the rise velocities to the liquid velocity, and the additional terms were used both in $k$ and $\varepsilon$ transport equations to account for the bubble-induced turbulence. They argued that, with an appropriate parameter in the bubble entrainment formula, the model is able to predict the main features of bubbly flows as evidenced by reasonable agreement with measured void fraction. Ma et al. (2011) incorporated a polydisperse two-fluid model into the VOF based Navier-Stokes solver TRUCHAS (Rider \& Kothe 1998). They proposed an entrainment model that connected bubble entrainment with turbulent dissipation rate, $\varepsilon$, at the air-water interface. The model was tested against the laboratory experimental data for an oscillatory bubble plume and the bubbly flow under a laboratory surf zone breaking wave using $2 \mathrm{D}$ simulations with a $k-\varepsilon$ turbulence closure in conjunction with the additional terms to account for bubble-induced turbulence. The exponential decay in time of void fraction observed in the laboratory experiments was captured by the model. The kinematics of the bubble plume, as 
well as the evolution of the bubble size spectrum over depth, were investigated. They employed a bubble breakup model proposed by Martínez-Bazán et al. $(1999 a, b)$ and showed that the model reproduced the $-10 / 3$ dependence for bubbles greater than the Hinze scale, consistent with the observation of Deane \& Stokes (2002). Ma (2012) and Kirby et al. (2012) extended the model to a LES framework with a constant Smagorinsky subgrid formulation for turbulence closure. They investigated surf zone breaking and found that the integrated void fraction has a linear growth and exponential decay in time. They showed that, as the vortices move downward, bubbles are transported to the lower part of the water column and concluded that the turbulent coherent structures tend to transport bubbles more deeply into the water column. Based on both the $2 \mathrm{D}$ and $3 \mathrm{D}$ simulations, they found that the presence of bubbles suppresses liquid phase turbulence and enstrophy.

Here, we extend an Eulerian-Eulerian polydisperse two-fluid model (Ma et al. 2011) to a LES framework with the dynamic Smagorinsky subgrid formulation for turbulence closure. To carefully validate the model against the detailed experimental studies as well as decrease the scale effects, the laboratory scale breaking waves imposed by a focussed wave packet is selected. In this paper, we concentrate on spanwise averaged quantities. The integral properties of the bubble plume, as well as the spanwise averaged mean and turbulent velocity fields during active breaking and their evolution, are compared to the available experiments. Dispersed bubble effects on the organized and turbulent motions and the different dissipation mechanisms are investigated. In addition, we examine the nonlinear interaction of different components in a wave packet. The 3D characteristics of the process, including wave induced coherent structures and their interaction with the entrained bubbles, are left for a subsequent paper.

In $\S 2$, the mathematical formulations and main assumptions are discussed. In $\S 3$, the corresponding experiments and mesh size selection for the $3 \mathrm{D}$ simulations are explained. In $\S 4$, the results of the $3 \mathrm{D}$ simulations are presented. Conclusions are given in $\S 5$. 


\section{Chapter 2}

\section{MATHEMATICAL FORMULATION AND NUMERICAL METHOD}

Lakehal et al. (2002) derived the filtered two-fluid model for a monodisperse bubbly flow. Using the multi-group approach explained by Carrica et al. (1999), bubbles are divided into $N G$ groups with a characteristic diameter, and the filtered polydisperse two-fluid model is derived based on the filtered monodisperse two-fluid model. In this section, we quickly review the traditional two-fluid model and work by Lakehal et al. (2002), and then the extension to the polydisperse two-fluid model and the corresponding main assumptions are discussed.

\subsection{The Filtered Two-Fluid Equations}

The filtered two-fluid model is obtained by applying a certain averaging process on the microscopic, instantaneous equations governing each phase evolving in the mixture. The conservation laws for each phase can be written using the phase indicator function $\chi(\mathbf{x}, t)$ at time $t$ and point $\mathbf{x}$, defined by (Drew 1983)

$$
\chi^{k}(\mathbf{x}, t)= \begin{cases}1 & \text { if } \mathbf{x} \text { lies in phase } k \text { at time } t \\ 0 & \text { otherwise }\end{cases}
$$

to determine volumes occupied by each phase. $k$ refers either to the gas phase or to the liquid phase. In the absence of heat and mass transfer, the continuity and momentum equations for each phase can be written as

$$
\begin{aligned}
& \frac{\partial}{\partial t}\left(\chi^{k} \rho^{k}\right)+\frac{\partial}{\partial x_{j}}\left(\chi^{k} \rho^{k} u_{j}^{k}\right)=0 \\
& \frac{\partial}{\partial t}\left(\chi^{k} \rho^{k} u_{i}^{k}\right)+\frac{\partial}{\partial x_{j}}\left(\chi^{k} \rho^{k} u_{i}^{k} u_{j}^{k}\right)=\chi^{k} \frac{\partial}{\partial x_{j}} \Pi_{i j}^{k}+\chi^{k} \rho^{k} g_{i}
\end{aligned}
$$


where $\rho^{k}$ is the phase density, $u^{k}$ is the phase velocity, and $g$ is gravitational acceleration. The phase net stress, composed of the pressure contribution, $p^{k}$, and the viscous stress $\sigma_{i j}^{k}$, is defined by $\Pi_{i j}^{k}=-p^{k} \delta_{i j}+\sigma_{i j}^{k}$. In a Newtonian fluid,

$$
\sigma_{i j}^{k}=\rho^{k} \nu^{k}\left(\frac{\partial u_{i}^{k}}{\partial x_{j}}+\frac{\partial u_{j}^{k}}{\partial x_{i}}\right)
$$

where $\nu^{k}$ is the phase kinematic viscosity.

Within the LES framework, a filtering process is utilized which is defined by

$$
\overline{f(\mathbf{x})}=\int_{D} G\left(\mathbf{x}-\mathbf{x}^{\prime} ; \Delta\right) f\left(\mathbf{x}^{\prime}\right) d^{3} \mathbf{x}^{\prime}
$$

where $D$ is the domain of the flow, $G\left(\mathbf{x}-\mathbf{x}^{\prime} ; \Delta\right)$ represents a spatial filter, and $\Delta$ is the filter width which should strictly be larger than the characteristic length scale of the dispersed phase. With this operator, the volume fraction of phase $k$ can be defined by

$$
\alpha^{k}(\mathbf{x})=\overline{\chi^{k}(\mathbf{x})}
$$

As carried out by Lakehal et al. (2002), the filtered equations are obtained by adopting a component-weighted volume-averaging procedure, in which

$$
\tilde{f}^{k}=\frac{\overline{\chi^{k} f^{k}}}{\overline{\chi^{k}}}=\frac{\overline{\chi^{k} f^{k}}}{\alpha^{k}} .
$$

By applying the above definition to (2.2) and (2.3) and ignoring surface tension effects, the filtered Eulerian-Eulerian equations are obtained (Lakehal et al. 2002),

$$
\begin{aligned}
& \frac{\partial}{\partial t}\left(\alpha^{k} \rho^{k}\right)+\frac{\partial}{\partial x_{j}}\left(\alpha^{k} \rho^{k} \tilde{u}_{j}^{k}\right)=0 \\
& \frac{\partial}{\partial t}\left(\alpha^{k} \rho^{k} \tilde{u}_{i}^{k}\right)+\frac{\partial}{\partial x_{j}}\left(\alpha^{k} \rho^{k} \tilde{u}_{i}^{k} \tilde{u}_{j}^{k}\right)=\frac{\partial}{\partial x_{j}} \alpha^{k}\left[\tilde{\Pi}_{i j}^{k}-\rho^{k} \tau_{i j}^{k}\right]+\alpha^{k} \rho^{k} g_{i}+\mathbf{M}^{k}
\end{aligned}
$$

where $\tilde{()}$ is the filter operation $(2.7), \mathbf{M}^{k}=\overline{\Pi_{i j}^{k} n_{j}^{k} \delta\left(x-x_{I}\right)}$ are the pure interfacial forces resulting from filtering, where $n_{j}^{k}$ is the normal unit vector pointing outward of 
phase $k, \delta$ is the Dirac distribution identifying the interface location with $x_{I}$, and

$$
\tau_{i j}^{k}={\widetilde{u_{i} u_{j}}}^{k}-\tilde{u}_{i}^{k} \tilde{u}_{j}^{k}
$$

is the subgrid scale (SGS) stress. Interphase momentum exchanges $\mathbf{M}^{k}$ and SGS stress $\tau_{i j}^{k}$ are the two unresolved terms in (2.9); our treatment of them will be explained in the following sections.

Equations (2.8) and (2.9) can be easily extended for the polydisperse two-fluid model by neglecting the momentum exchanges between bubble groups as in Carrica et al. (1999) and Ma et al. (2011). To simulate polydisperse bubbly flow, the dispersed bubble phase is separated into $N G$ groups. Each group has a characteristic bubble diameter $d_{k}^{b}, k=1,2, \cdots, N G$ and a corresponding volume fraction $\alpha_{k}^{b}$. By definition, the volume fraction of all of the phases must sum to one:

$$
\alpha^{l}+\sum_{k=1}^{N G} \alpha_{k}^{b}=1
$$

where superscripts $l$ and $b$ refer to liquid and bubble phase respectively. The volume fraction of the $k$ th bubble group is related to the bubble number density $N_{k}^{b}$ by

$$
\alpha_{k}^{b}=\frac{m_{k}^{b} N_{k}^{b}}{\rho^{b}}
$$

where $m_{k}^{b}$ is the mass of $k$ th bubble group, $N_{k}^{b}$ is number density of $k$ th bubble group and $\rho^{b}$ is the bubble density, which is assumed to be constant. The governing equations consist of mass conservation for liquid phase,

$$
\frac{\partial\left(\alpha^{l} \rho^{l}\right)}{\partial t}+\frac{\partial}{\partial x_{j}}\left(\alpha^{l} \rho^{l} \tilde{u}_{j}^{l}\right)=0
$$

momentum conservation for liquid phase,

$$
\frac{\partial\left(\alpha^{l} \rho^{l} \tilde{u}_{i}^{l}\right)}{\partial t}+\frac{\partial}{\partial x_{j}}\left(\alpha^{l} \rho^{l} \tilde{u}_{i}^{l} \tilde{u}_{j}^{l}\right)=-\frac{\partial}{\partial x_{j}}\left(\alpha^{l} \tilde{p}\right) \delta_{i j}+\alpha^{l} \rho^{l} g_{i}+\frac{\partial}{\partial x_{j}}\left[\alpha^{l}\left(\tilde{\sigma}_{i j}^{l}-\rho \tau_{i j}^{l}\right)\right]+\mathbf{M}^{g l}
$$


bubble number density equation for each bubble group

$$
\frac{\partial N_{k}^{b}}{\partial t}+\frac{\partial}{\partial x_{j}}\left(\tilde{u}_{k, j}^{b} N_{k}^{b}\right)=B_{k}^{b}+S_{k}^{b}+D_{k}^{b}, \quad k=1, \cdots, N G
$$

and momentum conservation for each bubble group,

$$
0=-\frac{\partial}{\partial x_{j}}\left(\alpha_{k}^{b} \tilde{p}\right) \delta_{i j}+\alpha_{k}^{b} \rho^{b} g_{i}+\mathbf{M}_{k}^{l g}, \quad k=1, \cdots, N G
$$

in which we neglect the inertia and shear stress terms in the gas phase following Carrica et al. (1999) and Ma et al. (2011). Here $\tilde{p}$ is filtered pressure which is identical in phases following the neglect of interfacial surface tension, $B_{k}^{b}$ is the source for the $k$ th bubble group due to air entrainment and $S_{k}^{b}$ is the intergroup mass transfer which only accounts for bubble breakup in the present study (Moraga et al. 2008, Ma et al. 2011). The bubble breakup model proposed by Martínez-Bazán et al. (1999a,b, 2010) is

employed. $D_{k}^{b}=\nu^{b} \frac{\partial N_{k}^{b}}{\partial x_{j}}$ stems from filtering the exact bubble number density equation and represents the SGS diffusion for the $k$ th bubble group with bubble difusivity, $\nu^{b}$, given by (2.31) below. $\mathbf{M}^{g l}$ and $\mathbf{M}_{k}^{l g}$ are the momentum transfers between phases, which satisfy the following relationship,

$$
\mathbf{M}^{g l}+\sum_{k=1}^{N G} \mathbf{M}_{k}^{l g}=0
$$

\subsection{Interfacial Momentum Exchange}

For a single particle moving in a fluid, the force exerted by the continuous phase on the particle includes drag, lift, virtual mass and Basset history forces. These forces are well established in the literature for both laminar and turbulent flows (Clift et al. (1978), Maxey \& Riley (1983) and Magnaudet \& Eames (2000), among many others). By neglecting the Basset history force, the filtered interfacial forces can be formulated as below (Lakehal et al. 2002),

$$
\mathbf{M}_{k}^{l g}=\tilde{\mathbf{f}}_{k}^{V M}+\tilde{\mathbf{f}}_{k}^{L}+\tilde{\mathbf{f}}_{k}^{D},
$$


where the filtered virtual mass force $\tilde{\mathbf{f}}_{k}^{V M}$, the filtered lift force $\tilde{\mathbf{f}}_{k}^{L}$ and the filtered drag force $\tilde{\mathbf{f}}_{k}^{D}$ are approximated as

$$
\begin{aligned}
& \tilde{\mathbf{f}}_{k}^{V M} \approx \alpha_{k}^{b} \rho^{l} C_{V M}\left(\frac{D \tilde{\mathbf{u}}^{l}}{D t}-\frac{D \tilde{\mathbf{u}}_{k}^{b}}{D t}\right) \\
& \tilde{\mathbf{f}}_{k}^{L} \approx \alpha_{k}^{b} \rho^{l} C_{L}\left(\tilde{\mathbf{u}}^{l}-\tilde{\mathbf{u}}_{k}^{b}\right) \times\left(\nabla \times \tilde{\mathbf{u}}^{l}\right) \\
& \tilde{\mathbf{f}}_{k}^{D} \approx \alpha_{k}^{b} \rho^{l} \frac{3}{4} \frac{C_{D}}{d_{k}^{b}}\left(\tilde{\mathbf{u}}^{l}-\tilde{\mathbf{u}}_{k}^{b}\right)\left|\tilde{\mathbf{u}}^{l}-\tilde{\mathbf{u}}_{k}^{b}\right|
\end{aligned}
$$

where $C_{V M}$ is the virtual mass coefficient with a constant value of $0.5, C_{L}$ is the lift force coefficient which is chosen as 0.5 and $C_{D}$ is the drag coefficient, which is given by (Clift et al. 1978)

$$
C_{D}= \begin{cases}\frac{24}{R e_{k}}\left(1+0.15 R e_{k}^{0.687}\right) & \text { for } \quad R e_{k}<1000 \\ 0.44 & \text { for } \quad R e_{k} \geq 1000\end{cases}
$$

where $R e_{k}=\frac{d_{k}^{b}\left|\tilde{\mathbf{u}}^{l}-\tilde{\mathbf{u}}_{k}^{b}\right|}{\nu^{l}}$ is the bubble Reynolds number of $k$ th group. Note that in pure water, with no contamination, the bubble drag coefficient is smaller than (2.20). As explained by Clift et al. (1978), the presence of surfactants, which is usually the case in laboratory condition and the real world, increases the drag force so that drag corresponds frequently to that of a solid sphere of the same size given by (2.20). Finally, an inherent assumption in (2.19) is that SGS effects on the interfacial forces are assumed to be negligibly small.

\subsection{Bubble Entrainment Model}

As already mentioned, a detailed examination of the process of bubble entrainment needs much more resolution than we have. Instead, dispersed bubbles are introduced into the water column using an entrainment model. Ma et al. (2011) correlated the bubble entrainment rate with the shear induced turbulence dissipation rate, $\varepsilon^{l}$, which is available in the RANS framework. In the present LES framework, we use the formulation of Ma et al. (2011) but change $\varepsilon^{l}$ to the shear induced production rate of SGS kinetic energy, $\varepsilon_{s g s, S I}^{l}$ (sometimes called the SGS dissipation rate) which 
represents the rate of transfer of energy from the resolved to the SGS motions, given by (2.30). For polydisperse bubbles, the formulation is

$$
B_{k}^{b}=\frac{c_{b}}{4 \pi}\left(\frac{\sigma}{\rho^{l}}\right)^{-1} \alpha^{l}\left(\frac{f\left(a_{k}\right) \Delta a_{k}}{\sum_{k=1}^{N G} a_{k}^{2} f\left(a_{k}\right) \Delta a_{k}}\right) \varepsilon_{s g s, S I}^{l},
$$

where $c_{b}$ is the bubble entrainment parameter and has to be calibrated in the simulation. $\sigma$ is surface tension, $a_{k}$ is the characteristic radius of each bubble group, $\Delta a_{k}$ is the width of each bubble group and $f\left(a_{k}\right)$ is the bubble size spectrum. As in Ma et al. (2011) and Shi et al. (2010), we use the size spectrum suggested by Deane \& Stokes (2002),

$$
f(a) \propto\left\{\begin{array}{lll}
a^{-10 / 3} & \text { if } \quad a>a_{h} \\
a^{-3 / 2} & \text { if } \quad a \leq a_{h}
\end{array}\right.
$$

where $a_{h}=1.0 \mathrm{~mm}$ is taken to be the Hinze scale, to distribute generated bubbles across the $N G$ bubble groups. Bubbles are entrained at the free surface cells if $\varepsilon_{s g s, S I}^{l}$ is larger than a critical value, $\varepsilon_{c}^{l}$, which is set to $0.01 \mathrm{~m}^{2} \mathrm{~s}^{-3}$.

\subsection{Subgrid-Scale Model}

The turbulent velocities in the continuous phase can arise from $(a)$ bubble agitations, e.g. turbulent wakes behind individual bubbles, and (b) large scale flow instabilities, e.g. shear induced instability (Fox 2012). In a continuous LES framework in which individual bubbles are not resolved and the filter width is in the inertial subrange, the main dissipative scales of motions are not resolved, and then transferring the energy from the resolved to SGS scales through shear- and bubble-induced dissipation should be modeled appropriately. The most widely used and simplest SGS model is the Smagorinsky model (Smagorinsky 1963) in which the anisotropic part of the SGS stress $\tau_{i j}^{l, d}$ is related to the resolved rate of strain,

$$
\tau_{i j}^{l, d} \equiv \tau_{i j}^{l}-\frac{\delta_{i j}}{3} \tau_{k k}^{l}=-2 \nu_{s g s}^{l} \tilde{\mathcal{S}}_{i j}^{l}
$$


where $\tilde{\mathcal{S}}_{i j}^{l}=\frac{1}{2}\left(\frac{\partial \tilde{u}_{i}^{l}}{\partial x_{j}}+\frac{\partial \tilde{u}_{j}^{l}}{\partial x_{i}}\right)$ is the resolved rate of strain and $\nu_{s g s}^{l}=\nu_{S I}^{l}+\nu_{B I}^{l}$ is the eddy viscosity of the SGS motions which is calculated using linear superposition of both the shear-induced, $\nu_{S I}^{l}$, and bubble-induced, $\nu_{B I}^{l}$, viscosities (Lance \& Bataille 1991). As in single phase flow, we take

$$
\nu_{S I}^{l}=\left(C_{s} \tilde{\Delta}\right)^{2}|\tilde{\mathcal{S}}|
$$

where $C_{s}$ is the Smagorinsky coefficient, $\tilde{\Delta}=(\Delta x \Delta y \Delta z)^{\frac{1}{3}}$ is the width of the grid filter and $|\tilde{\mathcal{S}}|=\sqrt{2 \tilde{\mathcal{S}}_{i j}^{l} \tilde{\mathcal{S}}_{i j}^{l}}$ is the norm of the resolved strain rate tensor.

The $C_{s}$ can be chosen as a constant $(0.1 \sim 0.2)$ or determined dynamically. Although the constant Smagorinsky model (CSM) is fairly good at fully turbulent flows with simple geometries (e.g. turbulent channel flow), it is too dissipative near the wall, in laminar and transition flows. A near-wall function can be used to give better behavior close to walls, but the extra dissipation can not be removed in transitional turbulence generated under breaking waves. In the case of deep water unsteady breaking, this is more important because we have a localized, unsteady TKE plume with relatively high intensity at the initial stage of the breaking, which gradually becomes more uniform and is mixed down to a greater depth. Shen \& Yue (2001) studied interaction between a turbulent shear flow and a free surface at low Froude numbers using single phase NavierStokes equations. The DNS results showed that the amount of energy transferred from the grid scales to the SGS reduced significantly as the free surface was approached. As a result, the coefficient $C_{s}$ should decrease towards the free surface (Shen \& Yue 2001, Figure 6a), which is not captured in CSM and leads to excessive dissipation near the free surface. The dynamic Smagorinsky models (DSM), on the other hand, provide a methodology for determining an appropriate local value for $C_{s}$, where turbulent viscosity converges to zero when flow is not turbulent and no special treatment is needed near the wall or in laminar and transitional regions. In addition, DSM is able to capture the anisotropy and the decrease of $C_{s}$ near the free surface as seen in DNS results.

In the present study, we use the dynamic procedure of Germano et al. (1991) 
with a least-square approach suggested by Lilly (1992) to compute $\left(C_{s}\right)^{2}$ based on double filtered velocities as

$$
\left(C_{s}\right)^{2}=-\frac{L_{i j} M_{i j}}{2 \tilde{\Delta}^{2} M_{i j} M_{i j}},
$$

where

$$
L_{i j}=\widehat{\tilde{u}_{i}^{l} \tilde{u}_{j}^{l}}-\widehat{\tilde{u}_{i}^{l}} \widehat{\tilde{u}}_{i}^{l} \text { and } M_{i j}=\alpha \widehat{2}|\widehat{\tilde{\mathcal{S}}}| \widehat{\tilde{\mathcal{S}}}_{i j}-\widehat{|\tilde{\mathcal{S}}| \tilde{\mathcal{S}}_{i j}}
$$

- represents the test scale filter with $\alpha=\widehat{\Delta} / \tilde{\Delta}>1$. We use the box filter given in Zang et al. (1993, Appendix A) with $\alpha=2$. As pointed out by Zang et al. (1993) and others, the locally computed values from (2.25) have large fluctuations and cause numerical instability especially in the case of negative diffusivity. To cope with this problem, averaging in a homogeneous direction (Germano et al. 1991, Vremen et al. 1997) or in a more general case, local averaging (Zang et al. 1993) should be applied. We perform local averaging and set negative values to zero as in Vremen et al. (1997).

The effect of SGS bubble-induced turbulence is added in the form of a bubbleinduced viscosity, $\nu_{B I}^{l}$, (Lance \& Bataille 1991, Fox 2012). We use the well known model proposed by Sato \& Sekoguchi (1975), given by

$$
\nu_{B I}^{l}=C_{\mu, B I} \sum_{k=1}^{N G} \alpha_{k}^{b} d_{k}^{b}\left|\tilde{\mathbf{u}}_{r, k}\right|
$$

where the model constant $C_{\mu, B I}$ is equal to 0.6 and $\tilde{\mathbf{u}}_{r, k}$ is the resolved relative velocity between the $k$ th bubble group and the liquid phase. Note that in regions of high void fraction, (2.27) may underestimate the bubble-induced viscosity due to bubble-bubble interactions, and then SGS pseudo-turbulent kinetic energy.

Using (2.4) and (2.23), the $\tilde{\sigma}_{i j}^{l}-\rho^{l} \tau_{i j}^{l}$ term in (2.14) can be written in the form of effective viscosity (Lakehal et al. 2002) as,

$$
\begin{aligned}
& \tilde{\sigma}_{i j}^{l}-\rho^{l} \tau_{i j}^{l}=\tilde{\sigma}_{i j}^{l}-\rho^{l}\left(\tau_{i j}^{l, d}+\frac{\delta_{i j}}{3} \tau_{k k}^{l}\right) \\
& \tilde{\sigma}_{i j}^{l}-\rho^{l} \tau_{i j}^{l, d}=2 \rho^{l} \nu_{e f f}^{l} \tilde{\mathcal{S}}_{i j},
\end{aligned}
$$


where

$$
\nu_{e f f}^{l}=\nu^{l}+\nu_{s g s}^{l}=\nu^{l}+\nu_{S I}^{l}+\nu_{B I}^{l} .
$$

The $\rho^{l} \frac{\delta_{i j}}{3} \tau_{k k}^{l}$ term can be absorbed in the pressure term. We write $\varepsilon_{s g s, S I}^{l}$ in (2.21) as

$$
\varepsilon_{s g s, S I}^{l}=2 \nu_{S I}^{l} \tilde{\mathcal{S}}_{i j} \tilde{\mathcal{S}}_{i j}=\nu_{S I}^{l}|\tilde{\mathcal{S}}|^{2}
$$

To compute $D_{k}^{b}$ in $(2.15)$, the bubble diffusivity, $\nu^{b}$, is given by

$$
\nu^{b}=\frac{\nu_{s g s}^{l}}{S c^{b}}
$$

where $S c^{b}$ is the Schmidt number for the bubble phase, taken equal to 0.7.

\subsection{Free Surface Tracking}

The VOF method with second-order piecewise linear interface calculation (PLIC) scheme (Rider \& Kothe 1998) is employed to track the free surface location. A linearity preserving, piecewise linear interface geometry approximation ensures that generated solutions retain second-order spatial accuracy. Second-order temporal accuracy is achieved by virtue of a multidimensional unsplit time integration scheme. In the VOF approach, an additional equation for fluid volume fraction $f$ is solved

$$
\frac{\partial f}{\partial t}+\nabla \cdot\left(\tilde{\mathbf{u}}^{l} f\right)=0
$$

where $f$ is the volume fraction of the water within a computational cell. If $f=1$, the cell is inside the water, while $f=0$, the cell is outside the water, otherwise, the cell is at the air(or void)-water interface and $f=0.5$ determines the position of the free surface.

\subsection{Boundary Conditions}

We do not solve the Navier-Stokes equations in any cell where $f=0$ and treat it as a void with zero density. Instead, the pressure remains unchanged and all of 
the velocity components are set to zero, which implies zero stress at the void-water interface. At the top boundary, the pressure is defined as zero and then the whole void area has zero pressure. As in Watanabe et al. (2005) and Christensen (2006), we ignore surface tension, which leads to homogeneous boundary conditions for shear and pressure at the free surface. A no-slip condition is imposed along the solid side walls and bottom. DSM gives zero turbulent viscosity near the wall and does not need any special treatment such as a near-wall damping function. A sponge layer is used to reduce wave reflection from the downstream boundary. At the inlet, the appropriate inflow condition is imposed. For dispersive wave cases, the wave packet was composed of 32 sinusoidal components of steepness $a_{i} k_{i}$ where the $a_{i}$ and $k_{i}$ are the amplitude and wave number of the $i$ th component. Based on linear superposition and by imposing that the maximum $\eta$ occurs at $x_{b}$ and $t_{b}$, the total surface displacement at the inlet can be obtained as (see $\mathbf{R M}$ )

$$
\eta(0, t)=\sum_{i=1}^{N=32} a_{i} \cos \left[2 \pi f_{i}\left(t-t_{b}\right)+k_{i} x_{b}\right]
$$

where $f_{i}$ is the frequency of the $i$ th component. $x_{b}$ and $t_{b}$ are the predefined location and time of breaking, respectively. The discrete frequencies $f_{i}$ were uniformly spaced over the band $\Delta f=f_{N}-f_{1}$ with a central frequency defined by $f_{c}=\frac{1}{2}\left(f_{N}-f_{1}\right)$.

Different global steepness, $S=\sum_{i=1}^{N=32} a_{i} k_{i}$ and $\Delta f / f_{c}$ lead to spilling or plunging breaking, where increasing $S$ and/or decreasing $\Delta f / f_{c}$ increases the breaking intensity (See Drazen et al. (2008) for more details). Free surface and velocities of each component are calculated using linear theory and then superimposed at $x=0$.

\subsection{Numerical Method}

The 3D VOF unstructured finite volume code TRUCHAS (Rider \& Kothe 1998) was extended to incorporate the polydisperse bubble phase (Ma et al. 2011) and different turbulent closures. The details of numerical method is given in (Ma et al. 2011). To summarize, the algorithm involves the following steps:

1. Material advection (the VOF model): The material interfaces are reconstructed using PLIC and interface normals are determined. Movement of the material 
between cells is based on combining the reconstructed geometry obtained from the PLIC algorithm with the normal component of fluid velocities located on the faces of all mesh cells.

2. Solve bubble number density and update the volume fractions: we use the bubble velocity at the previous time step to solve (2.15) and then update volume fractions obtained from (2.11) and (2.12).

3. Velocity prediction: The intermediate predicted velocities are calculated with updated volume fractions by a forward Euler step in time. This step incorporate explicit approximation to the momentum advection, body force and pressure gradient. These are updated in the correction step. Viscous forces are treated implicitly and then are averaged between the previous time step and the predicted step.

4. Pressure solution and velocity correction: The Poisson equation for pressure correction is solved using the preconditioned generalized minimal residual (GMRES) algorithm to satisfy solenoidal condition.

5. bubble velocity calculation: Using (2.16), the bubble velocities are calculated based on the updated fluid velocities.

\subsection{Reynolds Decomposition Of The Resolved Fields}

The Reynolds decomposition of any field variable, $\phi$, can be written as $\phi=<$ $\phi>+\phi^{\prime}$, where $<.>$ represents the ensemble averaged or organized flow, and $\phi^{\prime}$ is the turbulent fluctuation about this average. Similarly, for the resolved field variable, $\tilde{\phi}=\phi-\phi_{\text {sgs }}$, we can define $\tilde{\phi}=<\tilde{\phi}>+\tilde{\phi}^{\prime}$, then

$$
\phi^{\prime}=\phi-<\phi>=\tilde{\phi}+\phi_{s g s}-<\tilde{\phi}+\phi_{s g s}>=\tilde{\phi}^{\prime}+\phi_{s g s}-<\phi_{s g s}>
$$

where the SGS part is unresolved and its magnitude can only be estimated. Although ensemble averaging is practical in experimental studies, it is tedious in the numerical simulation due to the long computational times involved. The averaged variable in the homogenous direction (here the $y$-direction) can be interpreted as an organized motion, and the deviation from this average as the turbulent fluctuation. By this assumption, ensemble averaging actually is approximated by the spanwise averaging (Christensen \& Deigaard 2001), and enough grid points in the spanwise direction are 
needed to obtain a stable statistic. Christensen \& Deigaard (2001), Christensen (2006) and Lakehal \& Liovic (2011) used averaging on about 40 grid points in the spanwise direction to study turbulence under surfzone breaking waves, where lateral boundary condition were periodic. We use no slip boundary condition for the side walls and, because of wall effects, we should not do the averaging through the entire grid. We ignore 20 grid points near each wall, and then averaging is done on the remaining grid points

$$
<\tilde{\phi}(i, k)>\approx \tilde{\tilde{\phi}}(i, k)=\sum_{j=21}^{N_{y}-20} \frac{1}{N_{y}-40} \tilde{\phi}(i, j, k)
$$

where $N_{y}$ is the number of grid in the spanwise direction and () represents the spanwise averaging. Now, we can write

$$
\tilde{\phi}^{\prime}=\tilde{\phi}-\overline{\tilde{\phi}} \quad \text { and } \quad \tilde{\phi}_{\text {r.m.s. }}=\left[\overline{\tilde{\phi}^{\prime 2}}\right]^{1 / 2}
$$

where $\tilde{\phi}_{\text {r.m.s. }}$ is the resolved r.m.s. of turbulent fluctuations. In $\S 4.2$ we will show that (2.35) gives good results compared to the ensemble averaged measurements of RM. 


\section{Chapter 3}

\section{NUMERICAL SIMULATIONS}

We simulate all three cases in $\mathbf{L M}$ and two cases in $\mathbf{R M}$, where the dispersive focusing method was used to generate breaking. LM measured void fraction distribution and integral properties of the bubble plume and RM measured free surface evolution, ensemble averaged mean and turbulent velocities and estimated energy and momentum balance in the breaking region. Table 3.1 summarizes the input parameters of the simulated test cases, where $d$ is the quiescent water depth, and $t_{o b}$ and $x_{o b}$ are the time and location at which the forward-moving jet hits the undisturbed free surface, and are slightly different from the linear theory prediction of $t_{b}$ and $x_{b}$ defined in (2.33). The other parameters have been defined in $§ 2.6$. Besides the corresponding experiment in table 3.1, we also consider the void fraction measurements of Rojas \& Loewen (2010) and high resolution post breaking turbulence and velocity measurements by DM. The experimental set-ups in these two works are similar to the simulated cases, as summarized in table 3.2.

Unless otherwise indicated, the references for time, $x$ and $z$ directions are $t_{o b}$, $x_{o b}$ and still water level, respectively. This is consistent with the corresponding measurements and makes comparison easier. The normalized time, locations, velocities and vorticities then can be written as

$$
x^{*}=\frac{x-x_{o b}}{L_{c}}, \quad z^{*}=\frac{z-d}{L_{c}}, \quad t^{*}=\frac{t-t_{o b}}{T_{c}}, \quad u^{*}=\frac{u}{C_{c}}, \quad \omega^{*}=\omega T_{c} .
$$

where $T_{c}, L_{c}$ and $C_{c}$ are the period, wavelength and phase speed of the center frequency wave of the input packet, respectively. 
Table 3.1: Input parameters for the simulated cases

$\begin{array}{lccccccccc}\text { Case no. } & d(\mathrm{~m}) & S & f_{c}(1 / \mathrm{s}) & \Delta f / f_{c} & t_{b}(\mathrm{~s}) & x_{b}(\mathrm{~m}) & t_{o b}(\mathrm{~s}) & x_{o b}(\mathrm{~m}) & \text { Exp. } \\ \text { P1 } & 0.6 & 0.54 & 0.88 & 1.0 & 14.4 & 6.0 & 14.66 & 7.35 & \text { LM } \\ \text { P2 } & 0.6 & 0.45 & 0.88 & 1.0 & 14.3 & 6.7 & 14.44 & 7.58 & \text { LM } \\ \text { SP1 } & 0.6 & 0.38 & 0.88 & 1.0 & 14.3 & 6.7 & 14.68 & 7.85 & \text { LM } \\ \text { S1 } & 0.6 & 0.36 & 0.88 & 1.0 & 14.3 & 6.7 & 14.79 & 7.95 & \\ \text { P3 } & 0.6 & 0.352 & 0.88 & 0.73 & 20.5 & 8.46 & 19.04 & 8.35 & \text { RM } \\ \text { S2 } & 0.6 & 0.278 & 0.88 & 0.73 & 20.5 & 7.46 & 21.41 & 9.15 & \text { RM }\end{array}$

Table 3.2: Input parameters for additional experiments which are similar with the simulated cases

Similar Case no. $d(\mathrm{~m}) \quad S \quad f_{c}(1 / \mathrm{s}) \quad \Delta f / f_{c} \quad$ Exp.

$\begin{array}{llllll}\sim P 1 & 0.6 & 0.50 & 0.88 & 0.73 & \text { Rojas \& Loewen (2010) } \\ \sim P 3 & 0.6 & 0.36 & 1.08 & 0.75 & \end{array}$

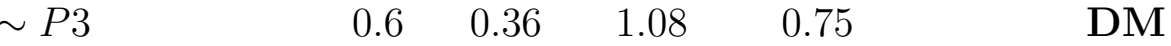

\subsection{Grid Size Selection For 3D Simulations}

In any LES, the goal is to model the small, dissipative scales of motion appropriately while avoiding extensive numerical computations that are needed for a DNS simulation. In turbulent bubbly flow, we also need to accurately model SGS effects induced by dispersed bubbles and interphase momentum exchanges. On the other hand, we must have sufficient mesh resolution to ensure that all relevant large scales of flow structures are captured.

\subsubsection{Free surface evolution}

In the first step, the grid size dependence study consists of comparison of the free surface evolution during the overturning process and splash-up, which can be performed using 2D simulations. Grid convergence analysis for an unsteady air/water interface in a turbulent bubbly flow is very difficult. Although much smaller 3D interface structures (Lubin \& Glockner 2013) can be resolved with higher mesh resolutions, the overall large 
scale dynamics of wave breaking are not affected by the small interface structures.

The refinement of grid spacing from $\Delta x=50 \mathrm{~mm}, \Delta z=16 \mathrm{~mm}$ to $\Delta x=$ $15 \mathrm{~mm}, \Delta z=4.6 \mathrm{~mm}$ (decreasing with a factor of $\sim 1.5$ ) is used for the most energetic plunger case, P1. Figure 3.1(a) shows that the jet tip becomes thinner and sharper as grid size decreases, and more details of the interface structure can be captured in the splash-up. The overall structure of the interface obtained with mesh resolutions $\Delta x=22.7 \mathrm{~mm}, \Delta z=7 \mathrm{~mm}$ and $\Delta x=15 \mathrm{~mm}, \Delta z=4.6 \mathrm{~mm}$ are similar. Figure 3.1(b) shows that both predict the surface elevation reasonably in comparison to the measurement especially during the overturning and first splash cycle $\left(t^{*}<0.4\right)$, where the process is less three dimensional. Although the initial cavity and splash-up can not be captured properly at the mesh resolution $\Delta x=33.3 \mathrm{~mm}, \Delta z=10.5 \mathrm{~mm}$, the free surface still has a comparable shape, and the increase of wave height in the splash cycle is captured. At further decrease of mesh resolution to $\Delta x=50 \mathrm{~mm}, \Delta z=16 \mathrm{~mm}$, the overall shape of the interface can not be resolved and free surface location drops considerably compare to the measurement. We may conclude that the first coarse mesh resolution is not adequate for LES and the appropriate resolution to capture the large scale interface structures would be between the second and third mesh resolution.

\subsubsection{Resolved turbulent fluctuations}

In the second step, we want to make sure that the mesh resolution is fine enough to resolve the main part of turbulent motions besides the large scale organized flow structures. In a successful LES with sufficiently fine resolution, at least $80 \%$ of the TKE should be resolved (Pope 2000). This criteria can be evaluated easily for each 3D simulation using (3.2) and (3.3). At high Reynolds number, with the filter width $(\tilde{\Delta})$ in the inertial subrange, the filtered velocity field accounts for nearly all of the kinetic

energy, $\langle\mathbf{E}>\approx<\tilde{\mathbf{E}}>\approx \overline{\tilde{\mathbf{E}}}$, and based on the mesh resolution some part of the TKE can be resolved. From (2.34), we can write

$$
\frac{1}{2}<u_{i}^{\prime} u_{i}^{\prime}>=\frac{1}{2}<\tilde{u}_{i}^{\prime} \tilde{u}_{i}^{\prime}>+\frac{1}{2}<u_{i}^{s g s} u_{i}^{s g s}>-\frac{1}{2}<u_{i}^{s g s}><u_{i}^{s g s}>-<\tilde{u}_{i}^{\prime} u_{i}^{s g s}>
$$



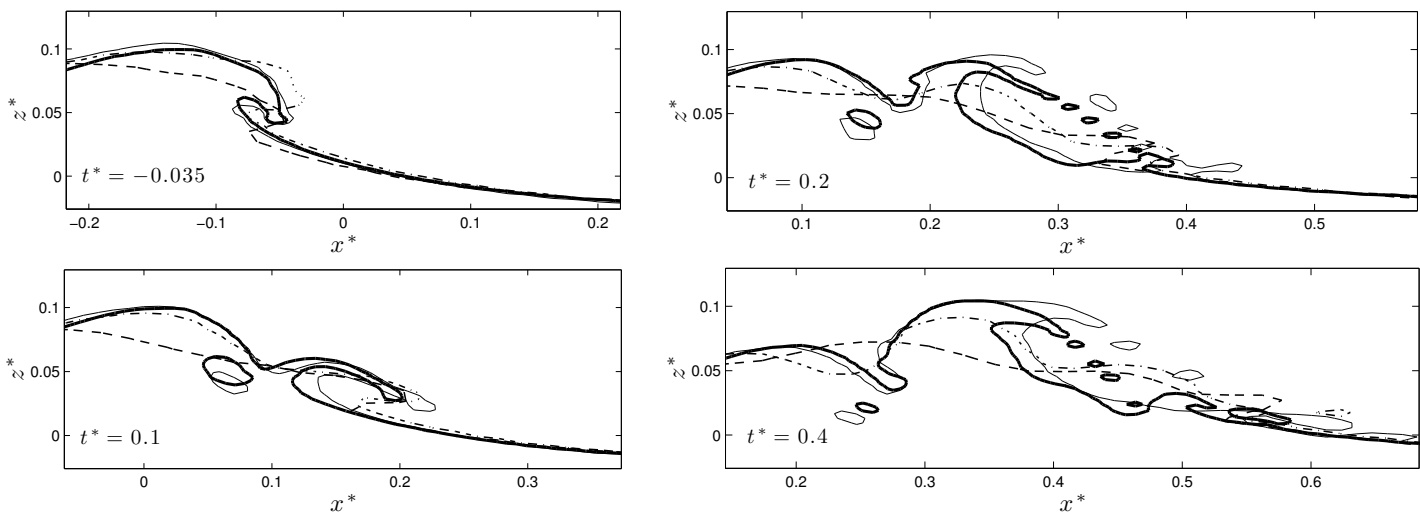

(a)

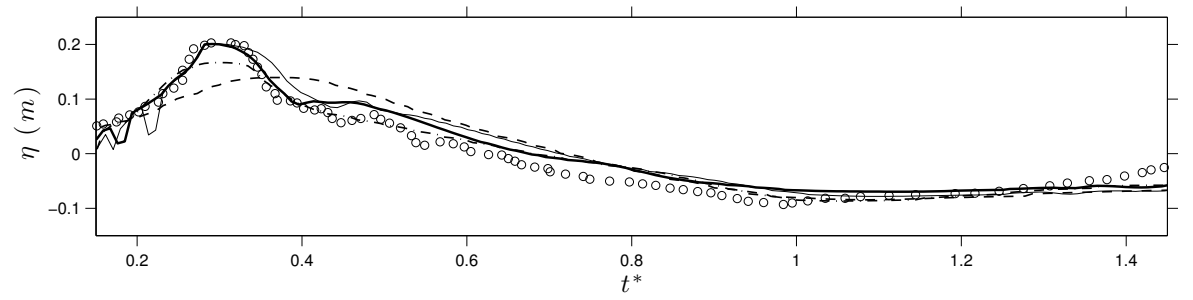

(b)

Figure 3.1: Grid dependence study of the free surface evolution (contours of $f=0.5$ ) during active breaking for P1. a) Snapshots of the overturning process and splash-up and $b$ ) Time history of the surface elevation at $x^{*}=0.285$. The different $2 \mathrm{D}$ mesh resolutions are $(\Delta x \times \Delta z),-15 \times$ $4.6 \mathrm{~mm},-22.7 \times 7 \mathrm{~mm},-\cdot-33.3 \times 10.5 \mathrm{~mm},---50 \times 16 \mathrm{~mm}$. Circles are the measurement and adopted from LM figure 1(a). 
The average of SGS fluctuations $\left\langle u_{i}^{s g s}>\right.$ is nearly zero, and we may neglect the correlation between resolved and SGS velocities. Now $<k>=\frac{1}{2}<u_{i}^{\prime} u_{i}^{\prime}>$ can be approximated by

$$
<k>\approx<\tilde{k}>+<k_{\text {sgs }}>\approx \tilde{\tilde{k}}+\bar{k}_{\text {sgs }}
$$

where $\tilde{k}=\frac{1}{2} \tilde{u}_{i}^{\prime} \tilde{u}_{i}^{\prime}$ is resolved TKE and $k_{s g s}=\frac{1}{2} u_{i}^{s g s} u_{i}^{s g s}$ is kinetic energy of SGS motions and can be estimated as (Pope 2000)

$$
k_{s g s}=\left[\frac{\nu_{s g s}}{C_{\nu} \tilde{\Delta}}\right]^{2}
$$

where $C_{\nu} \approx\left(\frac{3 C}{2}\right)^{-\frac{3}{2}} / \pi=0.094$ with the Kolmogorov spectrum constant, $C=1.5$. As we decrease the grid size (filter width) $k_{\text {sgs }}$ decreases and $\tilde{k}$ approaches to $k$.

We consider three 3D simulations for P1 with different mesh resolutions including $\Delta x=23.1 \mathrm{~mm}, \Delta y=7 \mathrm{~mm}, \Delta z=7 \mathrm{~mm}, \Delta x=34 \mathrm{~mm}, \Delta y=10.5 \mathrm{~mm}, \Delta z=10.5 \mathrm{~mm}$ and $\Delta x=50 \mathrm{~mm}, \Delta y=16 \mathrm{~mm}, \Delta z=16 \mathrm{~mm}$. The total spanwise averaged resolved

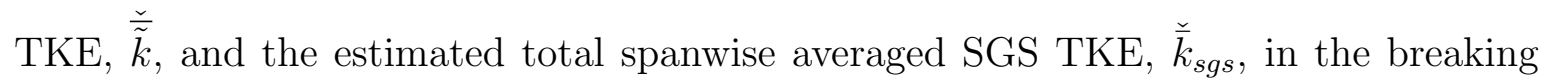
region can be defined as

$$
\check{\tilde{\tilde{k}}}=\int_{x_{1}^{*}}^{x_{2}^{*}} \int_{z_{1}^{*}}^{\eta^{*}} \overline{\tilde{k}} d z^{*} d x^{*}, \quad \check{\bar{k}}_{s g s}=\int_{x_{1}^{*}}^{x_{2}^{*}} \int_{z_{1}^{*}}^{\eta^{*}} \bar{k}_{s g s} d z^{*} d x^{*}
$$

where $x_{1}^{*}=-0.25, x_{2}^{*}=2.0$ and $z_{1}^{*}=-0.31$. Figure $3.2(\mathrm{a})$ shows that the ratio of $\frac{\check{\tilde{k}}}{\tilde{k}}$ to the estimated total spanwise averaged TKE, $\stackrel{\check{\tilde{k}}}{\check{\bar{k}}_{\text {sgs }}}$, is always more than $80 \%$ for the finest mesh resolution. Normalized $\check{\bar{k}}_{s g s}$ is shown in figure $3.2(\mathrm{~b})$ and decreases as mesh size decreases. Based on figure 3.2, we can conclude that the coarse resolution of $\Delta x=50 \mathrm{~mm}, \Delta y=16 \mathrm{~mm}, \Delta z=16 \mathrm{~mm}$ can only capture a small portion of the turbulent energy at the main dissipative period of breaking, $t^{*}=0 \sim 0.5$ (see $\S 4.8$ ) and the grid size between the two finest resolutions can reasonably capture a sufficiently large part of the turbulent fluctuations. 

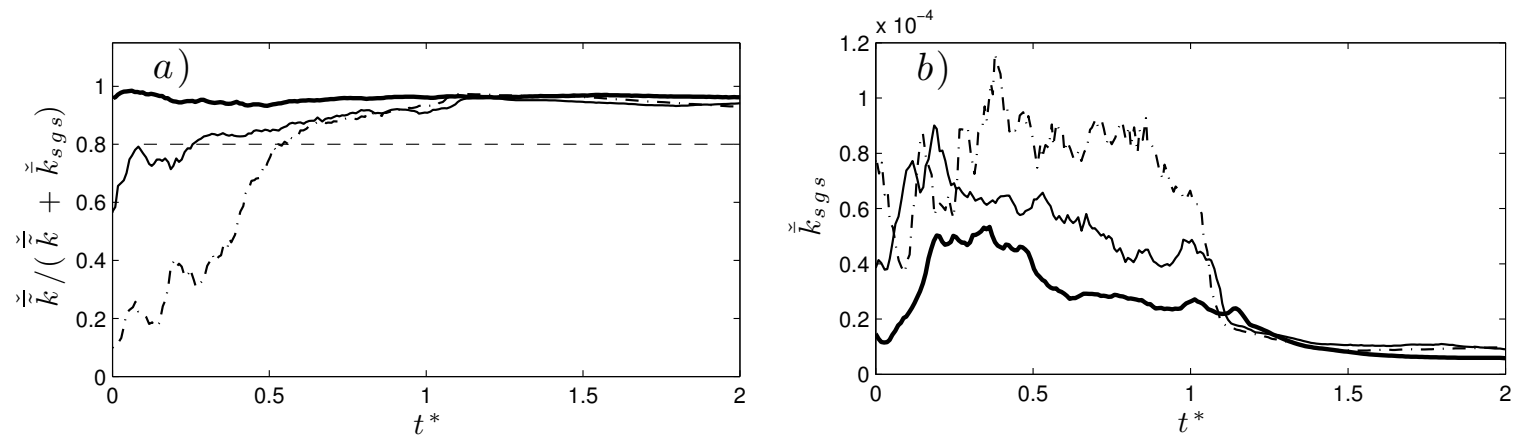

Figure 3.2: Grid dependence study of the resolved turbulent fluctuations for P1. a) total spanwise averaged resolved TKE, $\check{\tilde{\tilde{k}}}$ ratio to the total spanwise averaged TKE and $b$ ) normalized total spanwise averaged SGS TKE, $\check{\bar{k}}_{\text {sgs }}$. $\longrightarrow \Delta x=23.1 \mathrm{~mm}, \Delta y=7 \mathrm{~mm}, \Delta z=7 \mathrm{~mm},-\Delta x=34 \mathrm{~mm}, \Delta y=$ $10.5 \mathrm{~mm}, \Delta z=10.5 \mathrm{~mm},-\cdot-\Delta x=50 \mathrm{~mm}, \Delta y=16 \mathrm{~mm}, \Delta z=16 \mathrm{~mm}$. The reference value for $\check{\bar{k}}_{s g s}$ is $L_{c}^{2} C_{c}^{2}$.

\subsection{Model Set-Up}

Based on the 2D and 3D grid dependence studies for P1 in the previous section, the mesh resolution of $(\Delta x, \Delta y, \Delta z)=(23.1,7.0,7.0) \mathrm{mm}$ seems to capture the free surface evolution very well, and the estimate of $\check{\tilde{\tilde{k}}} / \check{\bar{k}}$ shows that more than 80 percent of total TKE in the breaking region is resolved. This procedure was repeated for the other cases and showed that this resolution is appropriate for the other smaller breakers. We thus choose this mesh resolution for the 3D simulations, as summarized in the table 3.3. Bubbles are divided into $N G=20$ groups with a logarithmic distribution of bubble sizes (similar to Ma et al. (2011)) where the maximum and minimum bubble diameters are taken as $8 \mathrm{~mm}\left(\tilde{\Delta} / d_{B}>1.3\right)$ and $0.2 \mathrm{~mm}$ (consistent with the observation by Deane \& Stokes (2002)), respectively. We used the same model parameters for all of the simulations, as summarized in table 3.4. All the 3D simulations are then repeated without the inclusion of dispersed bubble phase to examine the effects of dispersed bubbles on the organized and turbulent motions and energy dissipation. For simplicity, hereafter we drop () for all of the resolved variables. 
Table 3.3: Numerical set-up for the 3D LES simulated cases

$\begin{array}{lccc}\text { Case no. } & \text { Domain size }(\mathrm{m}) & \text { Mesh size } & \text { Mesh resolution }(\mathrm{mm}) \\ \text { P1 } & (15.0,0.63,0.864) & 650 \times 90 \times 124 & (23.1,7.0,7.0) \\ \text { P2 } & (15.0,0.63,0.864) & 650 \times 90 \times 124 & (23.1,7.0,7.0) \\ \text { SP1 } & (15.0,0.63,0.84) & 650 \times 90 \times 120 & (23.1,7.0,7.0) \\ \text { S1 } & (15.0,0.63,0.84) & 650 \times 90 \times 120 & (23.1,7.0,7.0) \\ \text { P3 } & (15.0,0.70,0.80) & 650 \times 100 \times 114 & (23.1,7.0,7.0) \\ \text { S2 } & (15.0,0.63,0.77) & 720 \times 90 \times 110 & (20.8,7.0,7.0)\end{array}$

Table 3.4: Model input parameters for the 3D LES simulations

Liquid density, $\rho^{l}$

Air density, $\rho^{b}$

Gravity, $g$

Water dynamic viscosity, $\mu^{l}$

Lift force coef. (2.18), $C_{L}$

Virtual mass coef. (2.18), $C_{V M}$

Entrainment parameter $(2.20), c_{b}$

Surface tension (2.20), $\sigma$

Test scale filter ratio $(2.25), \alpha$

Schmit number (2.30), $S c^{b}$

$1000 \mathrm{~kg} / \mathrm{m}^{3}$
$1.20 \mathrm{~kg} / \mathrm{m}^{3}$
$9.81 \mathrm{~m} / \mathrm{s}^{2}$
$10^{-6} \mathrm{~m}^{2} / \mathrm{s}$
0.5
0.5
0.36
$0.072 \mathrm{~N} / \mathrm{m}$
2
0.7

$1000 \mathrm{~kg} / \mathrm{m}^{3}$

$1.20 \mathrm{~kg} / \mathrm{m}^{3}$

$9.81-\mathrm{m} / \mathrm{s}^{2}$

0.5 


\section{Chapter 4}

\section{RESULTS}

\subsection{Bubble Entrainment And Transport}

\subsubsection{D free surface evolution and entrainment mechanisms}

In a plunging breaker, the finger-shape falling jet hits the forward face of the wave and both backward and forward splashes are formed. Based on the initial breaker intensity, the splash generation can be continued several times, and finally a bore-like region is formed and propagates downstream. In a spilling breaker, the jet and splashes are weak, and a bore-like front propagation is the main dominant feature. We can define three main entrainment mechanisms in a plunging breaker: cavity entrapment, jet/splash impacts and entrainment in the bore-like region, where, in a spilling breaker, the last one is the most important one. The entrained cavity will be fragmented into some large air pockets which may outgas very quickly or be further fragmented by turbulence into different bubble sizes down to the Hinze scale. Note that, under a 3D breaker, the air can escape laterally, leading to smaller cavity entrainment. To directly capture the details of these entrainment mechanisms we need to have very small spatial and temporal resolution that is intractable in 3D simulation of large laboratory scale events.

Figure 4.1 shows snapshots of the free surface evolution for the large plunging breaker, P1. It is clear that the model captures the overturning jet impact, splashup process and formation of a bore-like region. It can be seen that some void pockets captured by the VOF model at the first and second jet impacts seem to be fragmented to some extend and outgas because of buoyancy force. As we have explained in Appendix

A, if we have enough spatial resolution the volume of these void pockets initially are 
comparable to the volume of air entrapped by the overturning jet, but their evolution to smaller void pockets can be considered only as a crude approximation of actual air cavity break-up process. In $§ 4.1 .3$ we discuss more about these super-grid void pockets versus subgrid dispersed bubbles fed into the water column by the entrainment model. The finger shape structures (Saruwatari et al. 2009) can be seen in the forward splash $\left(t^{*}=0.05 \sim 0.3\right)$. In addition, comparing to $\mathbf{L M}$ figure $2 \mathrm{a}$, the time and location $\left(t^{*}=0.25, x^{*}=0.5\right)$ at which the forward splash hits the undisturbed free surface is very accurately captured by the model. When the jet hits the forward face of the wave, backward and forward splashes are generated and reach an elevation higher than the primary wave height. Local rise and depression of the surface (scars) is one of the typical features behind the progressive bore and can be seen in figure 4.1 for $t^{*}>0.9$, where the front bore is formed and propagates downstream $\left(x^{*}>0.9\right)$.

While large bubbles outgas very quickly, small bubbles are preferentially entrained into the coherent vortices generated during breaking and transported vertically by turbulent motions, and may remain in the water column for a very long time. Bubbles entrained by a plunging breaker can be divided into three different clouds. Figure 4.2 shows the 3D bubble plume evolution for $\mathrm{P} 1$, in which the two semicircular clouds are related to the two downbursts of turbulent motion under the first impacting jet and forward splash, and the third cloud represents bubbles entrained by the bore which are transported by vortices behind the bore. Figure 4.3 shows the results for the spilling/weakly plunging breaker, SP1. Because of the weak plunging and turbulent downburst, the third cloud is the dominant one and the 3D structures behind the progressive bore are more pronounced. The accumulation of bubbles near the side walls (especially during the outgassing phase) are consistent with the observation of LM. The detailed study of the large scale coherent vortices and their interactions with the entrained bubbles are left to a subsequent paper. Here we just want to emphasize that the entrainment processes are three-dimensional and bubble plume evolution is related to the large vortical structures. 


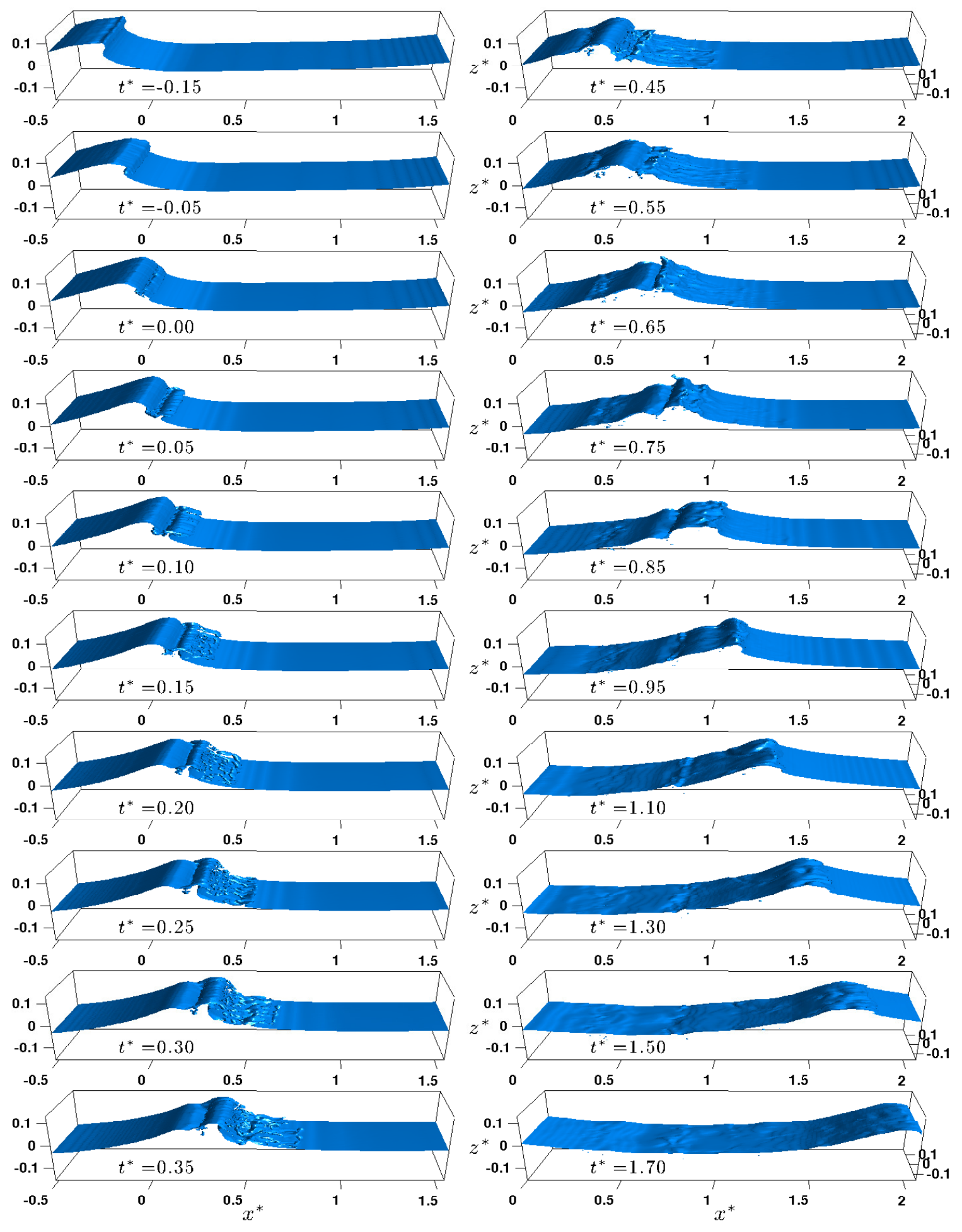

Figure 4.1: Snapshots of the free surface (isosurface of $f=0.5$ ) evolution for $\mathrm{P} 1$ 

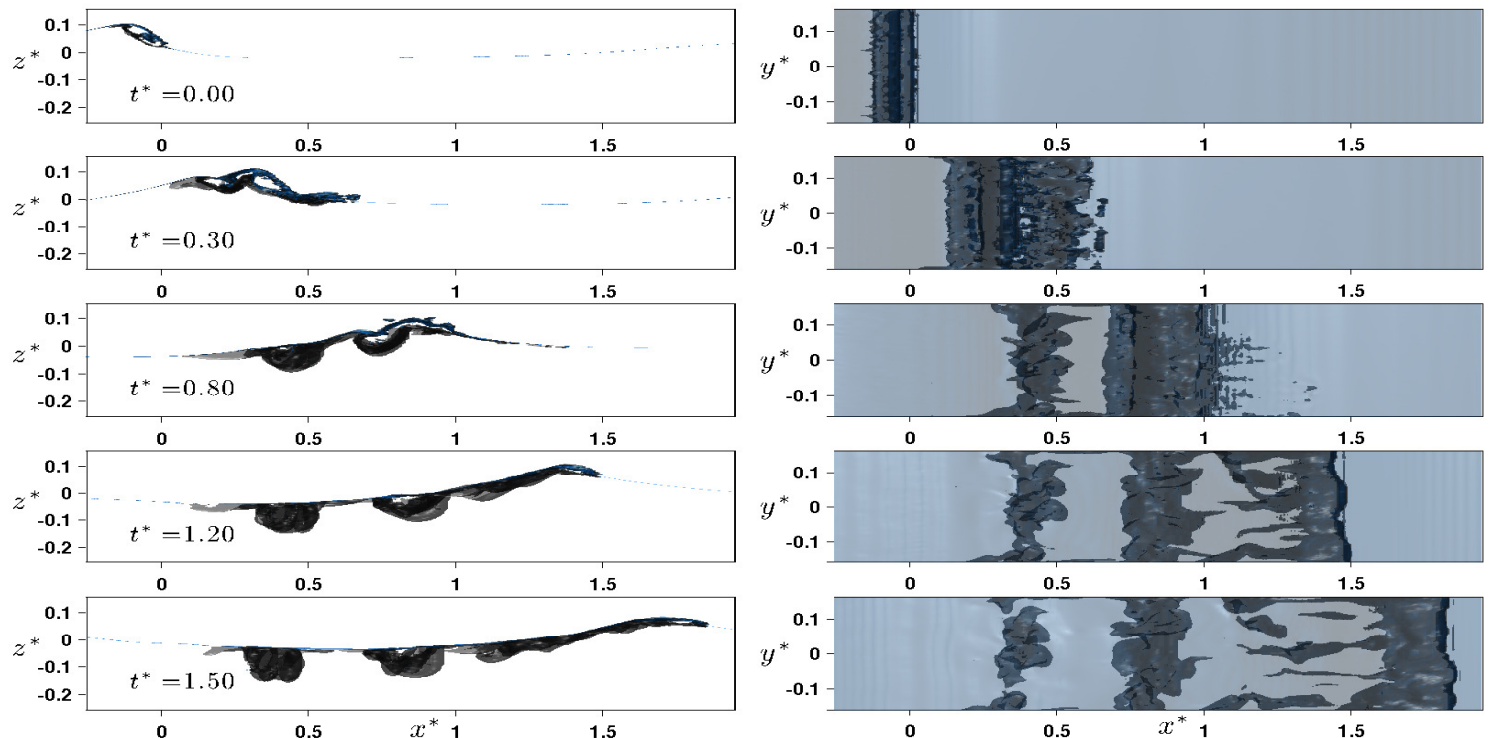

Figure 4.2: Snapshots of 3D bubble plume (isosurface of $\alpha^{b}=0.05 \%$ ) evolution in the breaking region for P1. (left) side view of the $3 \mathrm{~d}$ results and (right) top view of the $3 \mathrm{~d}$ results.
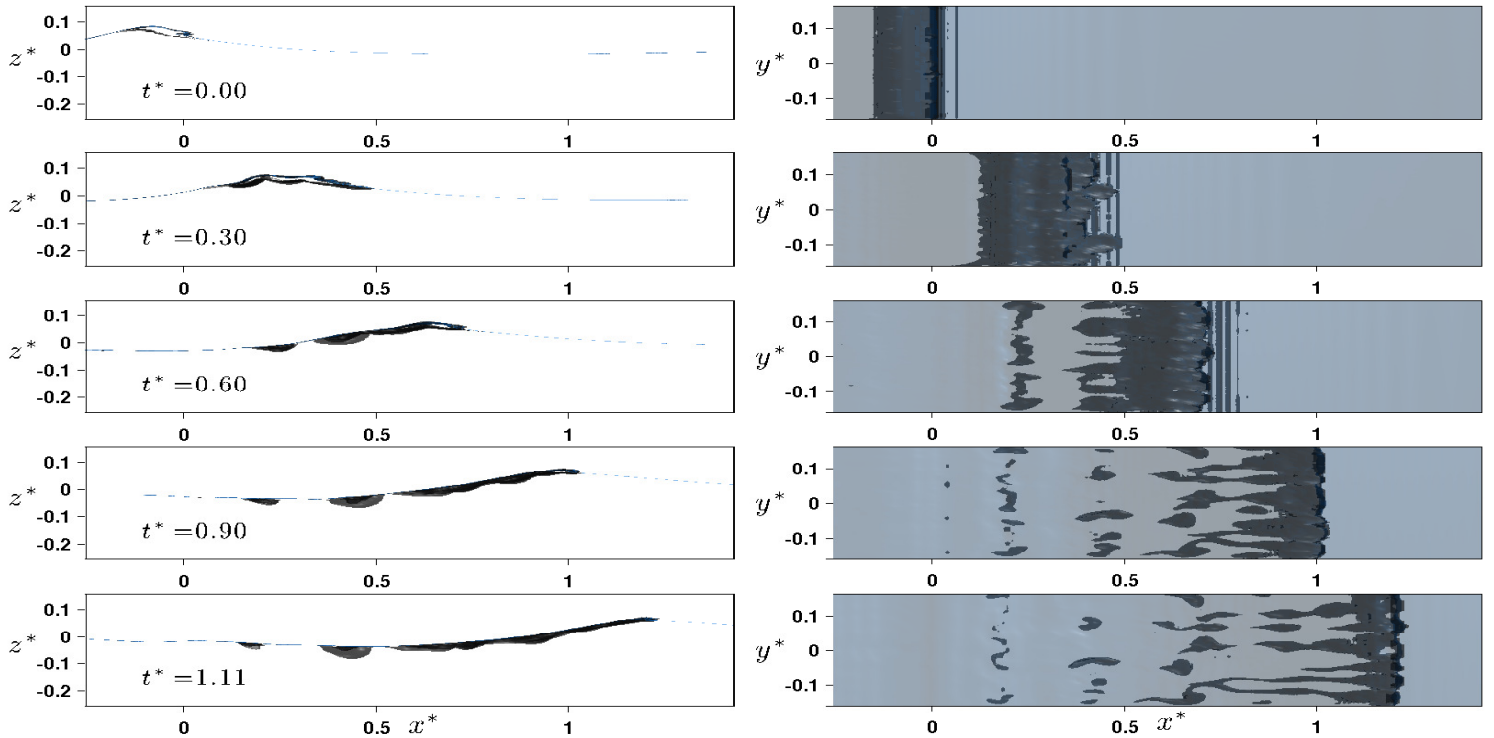

Figure 4.3: Snapshots of 3D bubble plume (isosurface of $\alpha^{b}=0.05 \%$ ) evolution in the breaking region for SP1. (left) side view of the $3 \mathrm{~d}$ results and (right) top view of the $3 \mathrm{~d}$ results. 

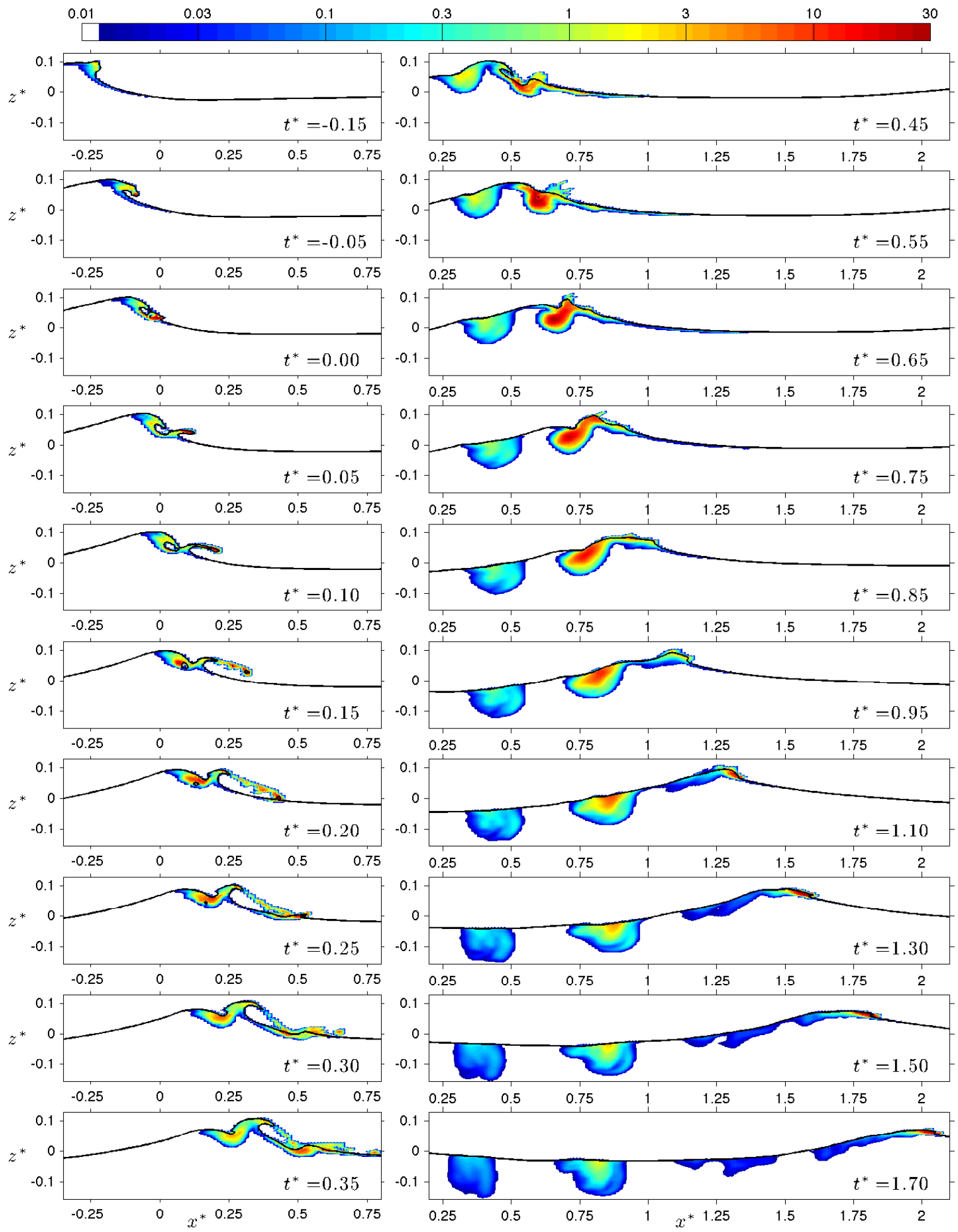

Figure 4.4: Time-dependent contour plots of the spanwise averaged void fraction distributions $\left(\bar{\alpha}^{b \%}\right)$ in the breaking region for $\mathrm{P} 1$. 

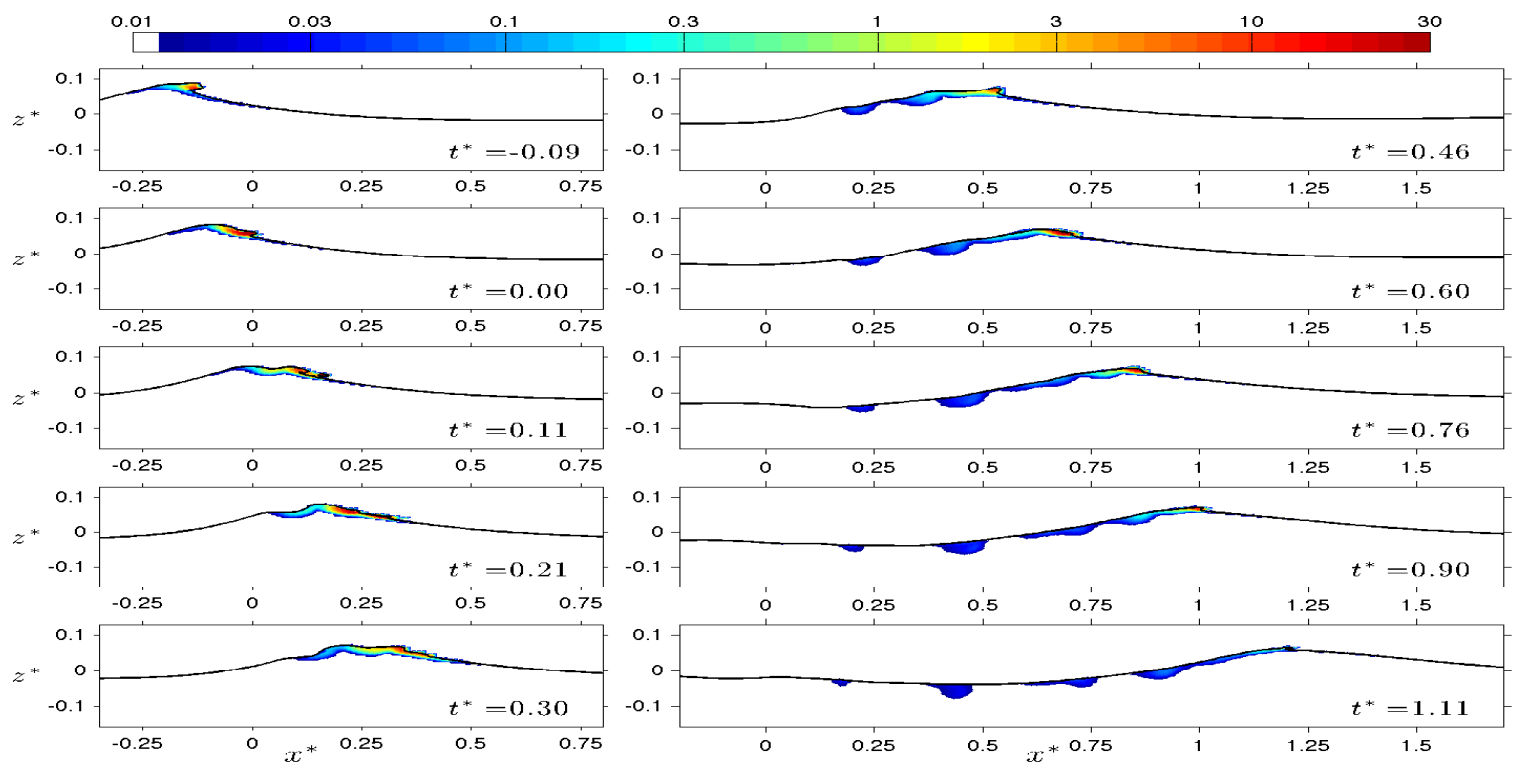

Figure 4.5: Time-dependent contour plots of the spanwise averaged void fraction distributions $\left(\bar{\alpha}^{b \%}\right)$ in the breaking region for SP1.

\subsubsection{Void fraction distributions}

Figure 4.4 shows snapshots of the spatial distribution of the spanwise averaged void fraction for P1. During cavity formation $\left(t^{*}=-0.15 \sim 0\right)$ the model predicts void fraction up to $10 \%$ at the jet toe, consistent with the measurement of Blenkinsopp \& Chaplin (2007, figure 4, a - c ) . The cavity entrapped by the jet entrains a considerable void volume during $t^{*}=0.0 \sim 0.25$, which is related to the region inside the black solid line, showing the spanwise averaged free surface location $(\bar{f}=0.5)$. The backward splash is formed between $t^{*}=0.2$ and $t^{*}=0.35$ and entrains some void volume. During $t^{*}=0.25 \sim 0.5$ the entrained cavity collapses and big void pockets outgas very quickly. Note that a cell with only void material, $f=0$, can collapse in a nonphysical manner. Although the break-up process can not be captured because of our spatial resolution as well as replacing actual air by void, the integrated rise velocity of the cavity can be captured reasonably well (see appendix A).

The primary semicircular bubble cloud initially advances approximately with the phase speed, but after $t^{*}=0.5$ its horizontal centroid becomes constant $\left(\sim x^{*}=\right.$ 
0.4) and then moves backward slowly after $t^{*}=1.1$. The secondary bubble cloud is generated by the impact of the forward splash during $t^{*}=0.25$ to $t^{*}=0.65$, where at $t^{*}=0.65$ we have the maximum entrainment by a jet-like impact similar to the primary jet. The spanwise averaged void fraction of the dispersed bubbles near the surface becomes more than $30 \%$ at $t^{*}=0.65$ and then decreases gradually to $\sim 1 \%$ at $t^{*}=1.7$. Similar behavior was reported by Rojas \& Loewen (2010) with slightly different wave packet conditions; see table 3.2. They observed the initial impact of the forward splash at $t^{*} \sim 0.32$ and maximum void fraction in the secondary bubble cloud at $t^{*} \sim 0.61$ in their plunging breaking case.

Figure 4.5 shows the results for SP1. There is a weak primary bubble cloud, and a very small cavity is captured by the VOF model. Instead of a forward splash, the bore front is formed at $t^{*}=0.45$ and air entrainment at the leading edge of the bore front becomes the dominant entrainment mechanism. The bubble plume advances with the front bore region and the maximum spanwise averaged void fraction is about 25\% near the leading edge. A similar pattern can be seen in P1 for $t^{*}>1.0, x^{*}>1.0$.

To quantify the distribution of the cavities captured by the VOF model versus the entrained dispersed bubbles, time averaged vertically integrated spanwise averaged volume of the dispersed bubbles, $\overline{\bar{V}}_{b}$, and the cavities, $\overline{\bar{V}}_{c}$, are calculated as

$$
\begin{aligned}
& \overline{\bar{V}}_{b}(x)=\frac{1}{t_{2}^{*}-t_{1}^{*}} \int_{t_{1}^{*}}^{t_{2}^{*}} \int_{z_{1}^{*}}^{\eta^{*}(t, x)} \bar{\alpha}^{b} d z^{*} d t^{*} \\
& \overline{\bar{V}}_{c}(x)=\frac{1}{t_{2}^{*}-t_{1}^{*}} \int_{t_{1}^{*}}^{t_{2}^{*}} \int_{z_{1}^{*}}^{\eta^{*}(t, x)}(1-\bar{f}) d z^{*} d t^{*}
\end{aligned}
$$

where $t_{1}^{*}=-0.25, t_{2}^{*}=2.0$ and $z_{1}^{*}=-0.31$. Figure 4.6 shows $\overline{\bar{V}}_{b}$ and $\overline{\bar{V}}_{c}$ for the different breakers. Because of the strong splashes in P1, a large secondary bubble cloud forms and leads to the peak in $\overline{\bar{V}}_{b}$ between $0.5<x^{*}<1.0$. In all of the breakers, when the bore front formed, uniform $\overline{\bar{V}}_{b}$ is predicted for about $0.5 L_{c}$ and then gradually decreases as the bore propagates further downstream and becomes less turbulent (figure 

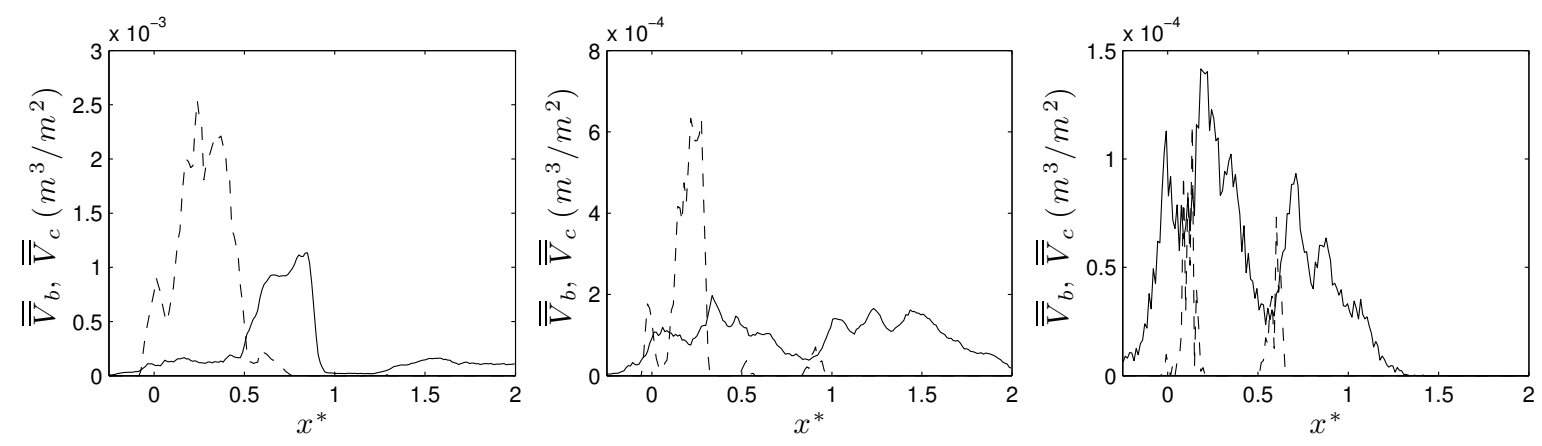

Figure 4.6: Time averaged vertically integrated spanwise averaged volume of the dispersed bubbles, $\overline{\bar{V}}_{b}$ and --- the cavities captured by the VOF model, $\overline{\bar{V}}_{c}$. (left) P1; (middle) P2 and (right) SP1.

$4.4, t^{*}=1.1$ to $t^{*}=1.7$ or figure $4.5, t^{*}=0.4$ to $\left.t^{*}=1.1\right)$.

\subsubsection{Integral properties of the bubble plume}

This section discusses some integral properties of the bubble plume. LM used $0.3 \%$ void fraction as a threshold to evaluate the integral properties of the bubble plume, using a conductivity probe on a $50 \times 50 \mathrm{~mm}$ grid. The measurements started a quarter of a period after breaking, and because of surface effects, they were based on measurements at depths below $2.5 \mathrm{~cm}$. The signals are ensemble averaged and then time averaged over $0.05 \mathrm{~s}$ intervals. They did not consider any upper limit for void fraction, which means initially the entrained cavities may lead to ensemble averaged void fractions more that $50 \%$. The total volume of the entrained dispersed bubbles, $V^{b}$, per unit length of crest are computed from

$$
V^{b}=\int_{A} \bar{\alpha}^{b} \mathcal{H}\left(\bar{\alpha}^{b}-\alpha_{t h l d}\right) d A
$$

where $\alpha_{\text {thld }}=0.3 \%$ is a threshold value and $\mathcal{H}$ is the Heaviside step function. The total cross sectional area of the dispersed bubble plume, $A^{b}$, are calculated as

$$
A^{b}=\int_{A} \mathcal{H}\left(\bar{\alpha}^{b}-\alpha_{\text {thld }}\right) d A
$$


The averaged volume fraction of the dispersed bubbles, $\alpha_{\text {ave }}^{b}$ are defined as

$$
\alpha_{\text {ave }}^{b}=\frac{V^{b}}{A^{b}}
$$

Finally, the horizontal and vertical centroids of the bubble plume, $\bar{x}^{b}, \bar{z}^{b}$, are calculated using

$$
\left(\bar{x}^{b}, \bar{z}^{b}\right)=\frac{\int_{A} \bar{\alpha}^{b} \mathcal{H}\left(\bar{\alpha}^{b}-\alpha_{\text {thld }}\right)(x, z) d A}{A^{b}} .
$$

As explained by LM, the total volume of the bubble plume and the centroid positions reach their asymptotic values for the threshold values equal to $0.3 \%$ or less. However, the cross-sectional area and the averaged volume fraction do not reach their respective asymptotic values. The regions with void fraction close to the threshold value contribute significantly to the cross-sectional area of the bubble plume, but do not contribute much to the total volume of air. Thus, the total volume of the bubble plume predicted by the model can be expected to have more correlation with the measurement, in comparison to the plume area or averaged volume fraction. On the other hand, predicted cross-sectional area and averaged volume fraction should have a similar pattern, while the absolute values may be different.

Figures 4.7 and 4.8 show the time-dependent averaged volume fraction and normalized volume and cross-sectional area of the bubble plume for P1 and SP1, respectively. $V_{0}$ is the total volume of air per unit length of crest enclosed by the forward jet as it impacts the free surface, as seen from video images taken from the side wall of the experimental channel for P1, and is the maximum of the measured volume of the entrained air for SP1. Using the measured $V_{0}\left(0.0098\right.$ and $0.0025 \mathrm{~m}^{3} / \mathrm{m}$ for P1 and SP1, respectively) makes it possible to compare the absolute values between the model and measurement. During $t^{*}=0 \sim 0.5$, the total volume of the entrained dispersed bubbles (solid line in figures $4.7(\mathrm{a})$ and $4.8(\mathrm{a})$ ) accounts for less than $10 \%$ in $\mathrm{P} 1$ to about $40 \%$ for SP1. The reason is that the entrainment model correlates the volume of 

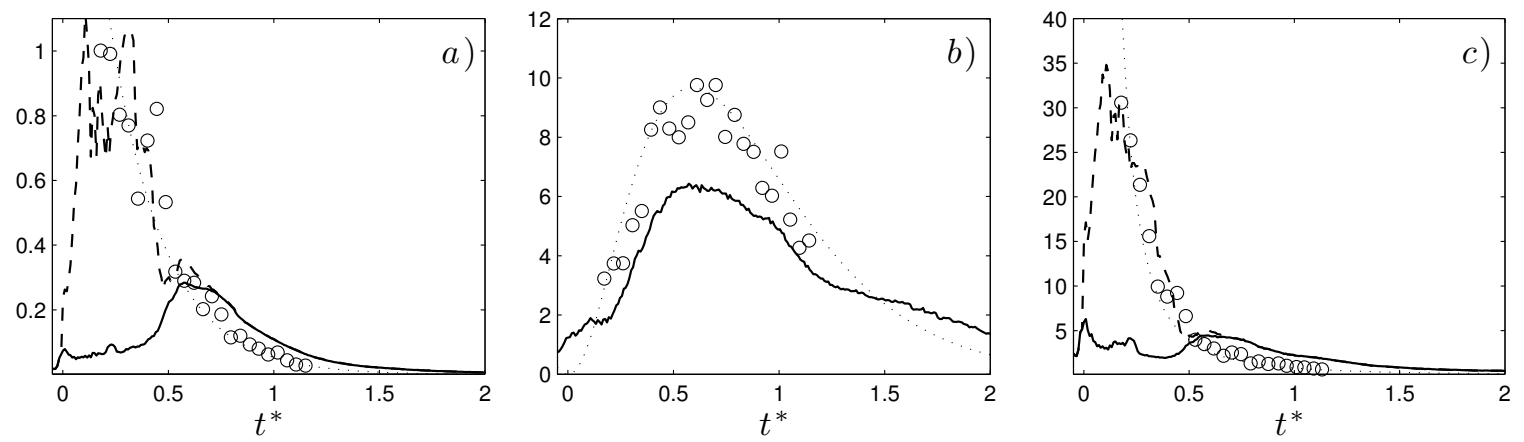

Figure 4.7: Integral properties of the bubble plume for P1. a) Normalized volume of the entrained air, $\left.-V^{b} / V_{0},--\left(V^{b}+V^{c}\right) / V_{0} ; b\right)$ normalized bubble plume area $-A^{b} / V_{0}$, and c) averaged volume fraction (\%), $\alpha_{\text {ave }}^{b},---\left(V^{b}+V^{c}\right) / A^{b}$. Total volume of the cavities captured by the VOF model is $V^{c}=\int_{A}(1-\bar{f}) d A$. $\quad \cdots \cdots$ Experimental fitted curves from LM. Circles are the measurements of the corresponding case adopted from LM figure 3.
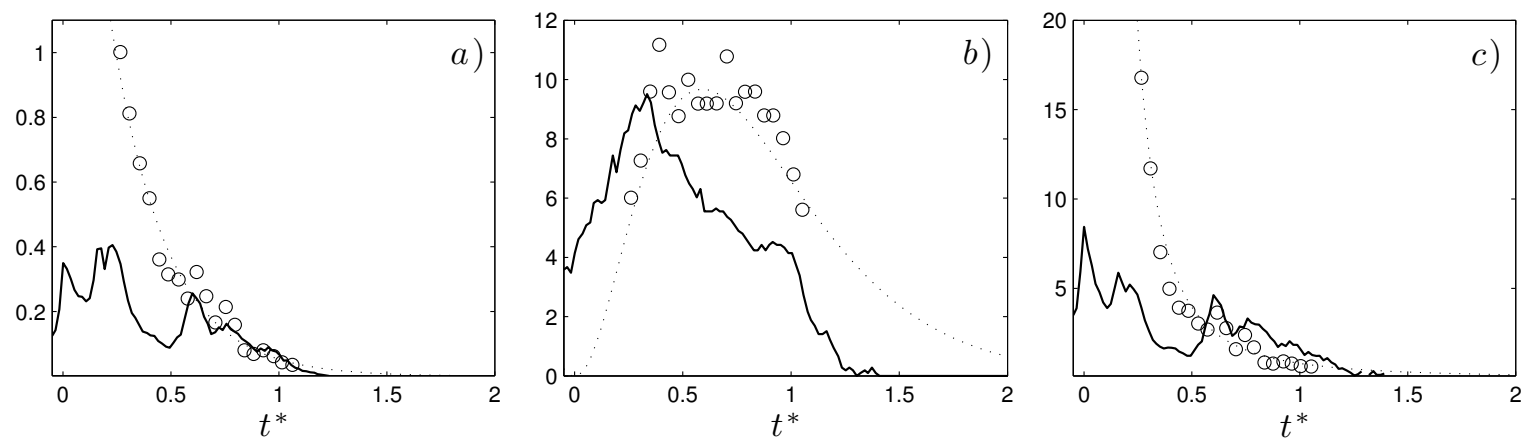

Figure 4.8: Integral properties of the plume for SP1. Definitions are the same as figure 4.7
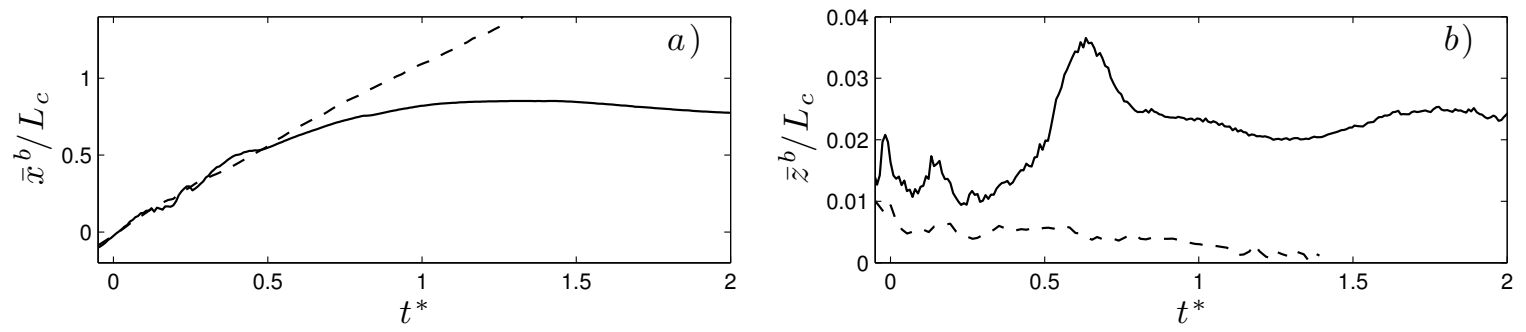

Figure 4.9: Normalized centroid positions of the bubble plume for $\_$P $1, \quad---$ SP1. 
the entrained bubbles (see (2.21)) with shear induced SGS production rate, $\varepsilon_{s g s, S I}$, near the free surface, and thus, can not capture the entrainment due to the cavity entrapped by the overturning jet, which is not a turbulence dependent mechanism but relates to the geometry of the breaker. Figure 4.6(a) shows that the total volume based on both the dispersed bubbles fed in by the entrainment model and the cavities captured by the VOF model (dash line in figures 4.7(a)) compare well to the measurements for P1. For both cases, the entrainment model predicts the correct volume of entrained bubbles after $t^{*}=0.6$, when the turbulence and leading edge entrainment mechanisms in the splash-up and bore-like region become dominant, while the air pockets and/or bigger bubbles outgas very quickly because of the larger rise velocity and smaller initial penetration depth. As expected, the results for SP1 is more comparable to the measurement during $t^{*}=0 \sim 0.5$, which is because of the smaller cavity entrapment.

The plume area and averaged volume fraction are also predicted reasonably well. Similar to the plume volume if we consider the cavities, the averaged volume fraction becomes comparable with the measurement during $t^{*}=0 \sim 0.5$ in P1. The general trend of plume area evolution is also consistent with the measurement of Callaghan et al. (2013, figures 4 and 5).

Figure 4.9(a) shows that the entrained bubbles are initially transported at the characteristic phase speed, $C_{c}$, in both breakers. In P1, because of the wave-induced strong downburst type large vortices which do not propagate downstream, the primary and secondary bubble clouds have nearly fixed positions after $t^{*}=0.75$, and as a result $\bar{x}^{b}$ becomes nearly constant. In $\mathrm{P} 1$, the vertical centroid position of the plume (figure 4.9(b)) clearly shows a peak at the second jet/splash impact which is correspond to the secondary bubble clouds. Then, it becomes nearly constant at later times. As observed by the experiments, there is also a peak $\bar{z}^{b}$ during the first jet impact which can not be captured by the entrainment model. In SP1 $\bar{z}^{b}$ gradually decreases, and because of no significant downburst, which is the case for the small breakers and spilling type whitecaps, the bubble plume is more concentrated near the free surface. 

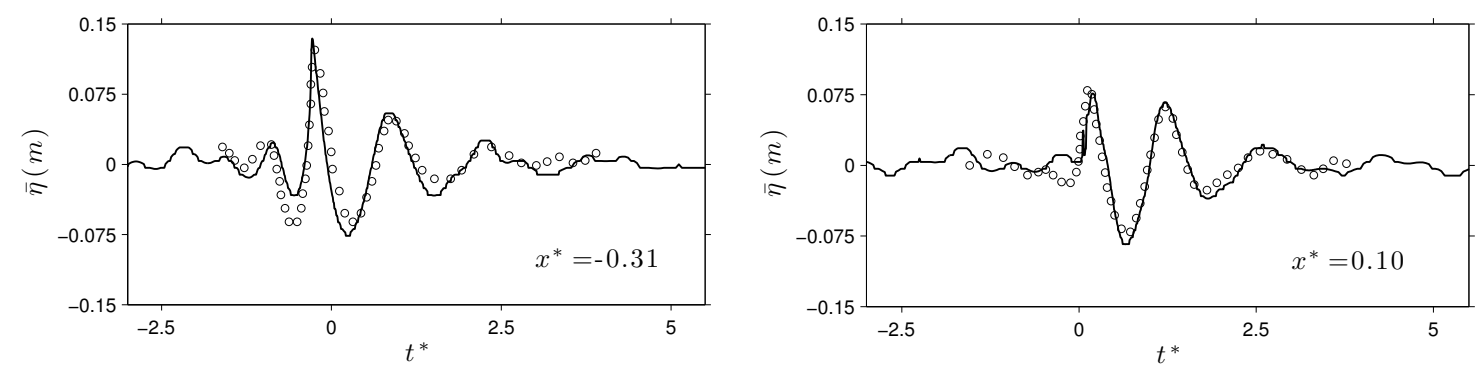

Figure 4.10: Free surface time series near the breaking point for P3. Circles are the measurements of the corresponding case adopted from RM figure 7(a).

\subsection{Nonlinear Interaction Of Wave Packet Components}

The input packet consists of short waves followed by long waves. As they propagate towards the predefined focal point, the packet becomes steeper, and the surface evolves toward breaking. Figure 4.10 shows the spanwise averaged free surface, $\bar{\eta}$, near the break point for P3. The considerable drop in peak $\bar{\eta}$ results from plunging breaking occurring at $x^{*}=0$. Compared to the measurements, the model captures the time dependent free surface location before and after the break point fairly well. To study frequency evolution as the packet approaches the focal point and understand which frequencies in the packet lose energy during breaking, $\mathbf{R M}$ measured the power spectrum based on a single measurement of the free surface at different locations. They showed that near the wave maker the wave packet is similar to its theoretical 'top hat' shape, and as it evolves to the focal point, loses its 'top hat' shape, with energy spread to higher frequencies. They observed that breaking occurred between $k_{c}\left(x-x_{b}\right)=-5$ and 0 where $k_{c}=2 \pi L_{c}^{-1}$, and that the loss in the high frequency end of the first harmonic band and in the second harmonic band was considerable. Figure 4.11 shows the power density spectra of the wave packet for the incipient breaking case $(S=0.257)$, and P3 based on the FFT of the spanwise averaged free surface time series which are smoothed using 5-point band averaging. The dashed line corresponds to the spectra at $k_{c}\left(x-x_{b}\right)=-15$ for each column. The deviation from the upstream spectra clearly shows the above mentioned processes, which were previously observed in RM figure 

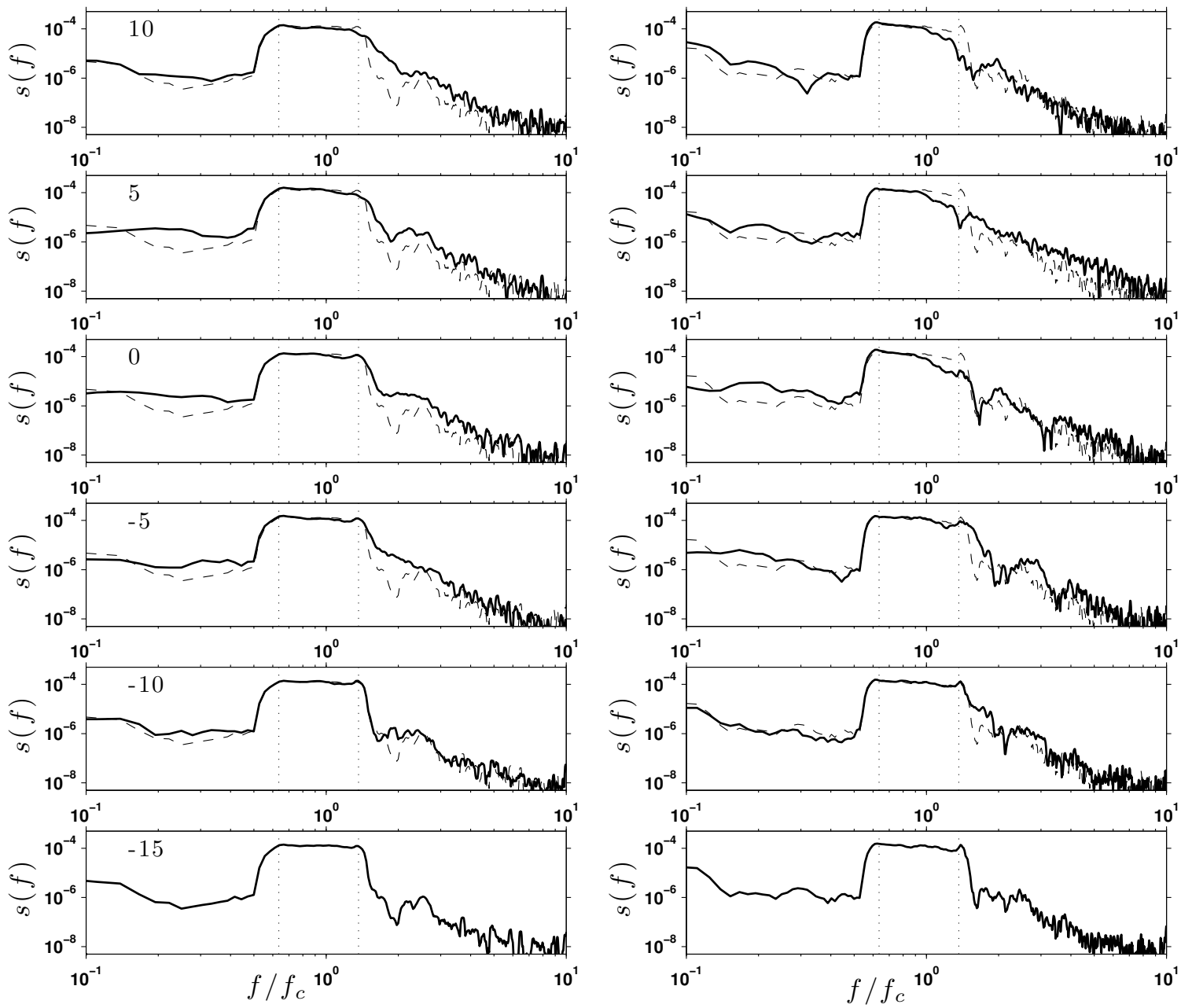

Figure 4.11: Power density spectra of the wave packet for (left) incipient breaking case, $S=0.257$ and (right) P3. The numbers on the graphs are the values of $k_{c}\left(x-x_{b}\right)$. The dash line corresponds to the spectra at $k_{c}(x-$ $\left.x_{b}\right)=-15$ for each column. Vertical dotted lines show the frequency band boundaries of the input wave packet. 

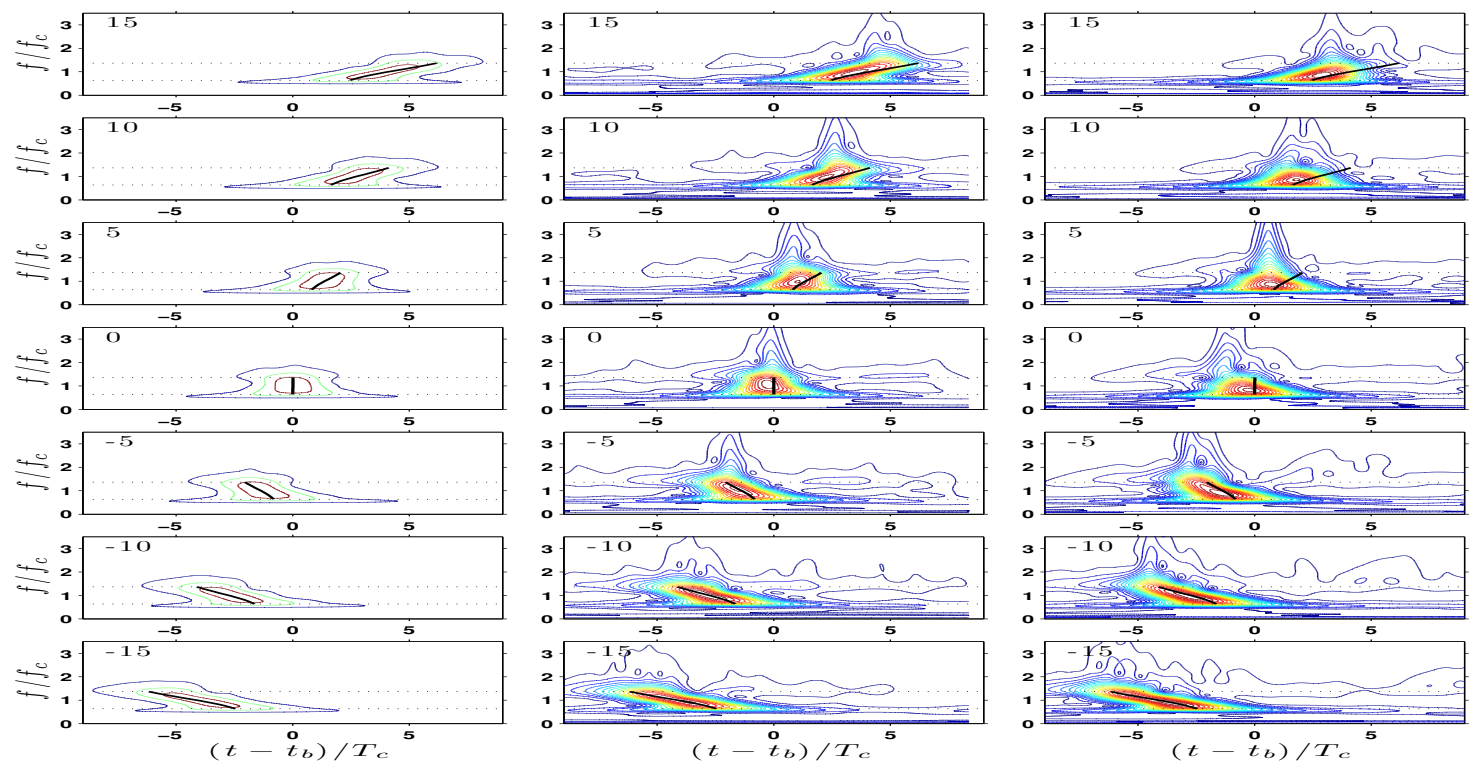

Figure 4.12: Modulus of the wavelet transform of (left) the linear packet, $S=0.05$; (middle) incipient breaking and (right) P3. The numbers on the graphs are the values of $k_{c}\left(x-x_{b}\right)$. Horizontal dotted lines show the frequency band boundaries of the input wave packet. — shows the arrival time of the different frequencies based on the linear theory group velocity.
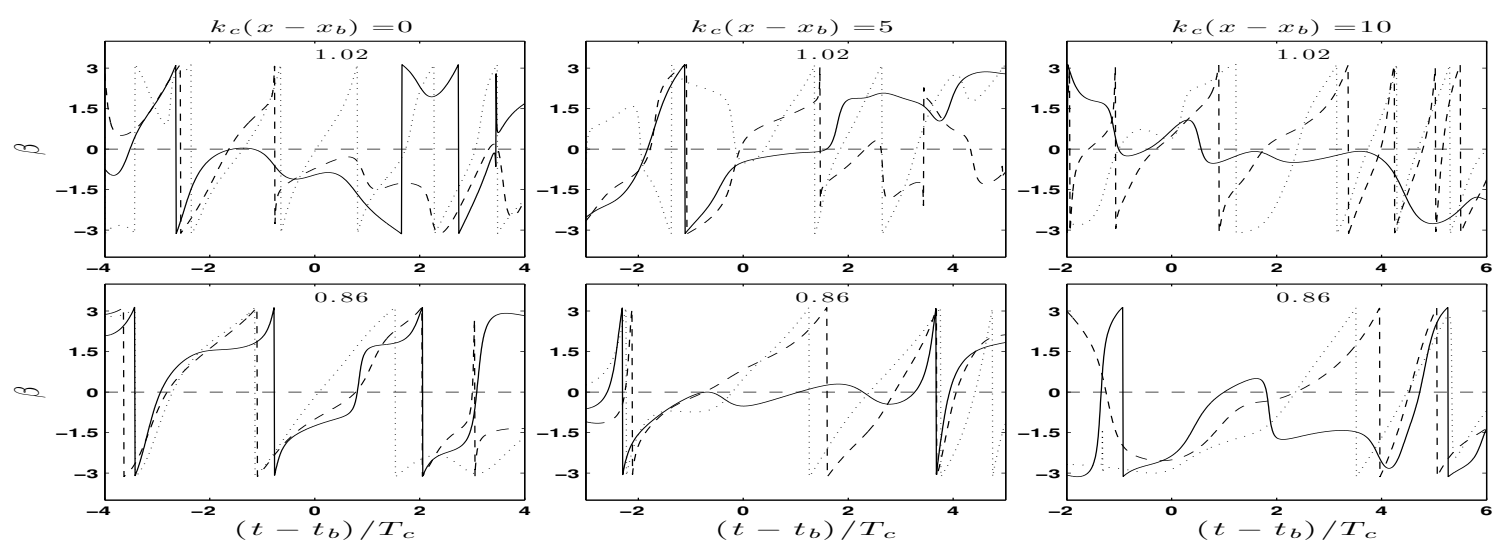

Figure 4.13: Phase angle, $\Theta_{B_{\bar{\eta}}}$, of the spanwise averaged free surface bispectrum $\left.B_{\bar{\eta}}\left(s_{1}, s_{1}, t\right)\right)$, for $\cdots \cdots$ linear packet, $S=0.05$; - - incipient breaking and $-\mathrm{P} 3$. The numbers on the graphs are the values of $f_{1} / f_{c}$, where $f_{1}=1 / c s_{1}$ and $c=4 \pi /\left(\omega_{0}+\sqrt{2+\omega_{0}^{2}}\right)$ is a constant. 
25 and 26.

We next use the continuous wavelet transform to determine the spatio-temporal structure and relative phasing of frequency components in the single breaking wave. The continuous wavelet transform $W_{\bar{\eta}}$ of a discrete sequence of the spanwise averaged free surface time series at certain location, $\bar{\eta}(t)$, is defined as the convolution of $\bar{\eta}(t)$ with a scaled and translated version of a mother wavelet,

$$
W_{\bar{\eta}}(s, t)=\frac{1}{\sqrt{s}} \int_{-\infty}^{\infty} \bar{\eta}(\tau) \phi^{\star}\left(\frac{\tau-t}{s}\right) d \tau
$$

where $t$ is time, $s$ is the scale factor, $\tau$ is the translation factor, $\sqrt{s}$ is for energy normalization across different scales, and ${ }^{\star}$ denotes the complex conjugate. The wavelet kernel adopted here is the Morlet wavelet and is expressed as

$$
\phi(t)=e^{i \omega_{0} t} e^{-\frac{t^{2}}{2}}
$$

where $\omega_{0}$ is the non-dimensional central frequency of the analyzing wavelet. The wavelet transform is computed in Fourier space to obtain an arbitrary number and distribution of scales.

The modulus of the wavelet transform of a linear packet, incipient breaking and P3 are shown in Figure 4.12. In the linear case, the results follow the linear theory prediction in which all the frequencies in the packet arrive at the predefined focal point, $x_{b}$, at the predefined time, $t_{b}$ (Note that in the figure the numbers on top left corner are values of $\left.k_{c}\left(x-x_{b}\right)\right)$. In addition, energy at each frequency component propagates with its corresponding group velocity before and after the focal point, which leads to symmetry of the results about $k_{c}\left(x-x_{b}\right)=0$. In the incipient breaking case and P3, as the packet approaches the focal point, nonlinear effects lead to faster propagation of the energy, generating a permanent lead in arrival time relative to the linear prediction. Although this lead in arrival time exists in both nonlinear cases, in P3 there is a phase locking after breaking and nearly all of the frequencies propagate together between 
$0<k_{c}\left(x-x_{b}\right)<10$. Strictly speaking, phase coupling occurs if two frequencies, say $f_{1}, f_{2}$, are simultaneously present in the signal along with their sum frequency, $f_{3}=f_{1}+f_{2}$, with $\Theta_{3}=\Theta_{1}+\Theta_{2}+$ const where $\Theta_{i}$ is a corresponding phase of $f_{i}$. This phase coupling process after breaking is further demonstrated by looking at the first higher-order spectrum, or bispectrum, of the wavelet transform near the peak frequency of the wave packet. The bispectrum is defined as

$$
B_{\bar{\eta}}\left(s_{1}, s_{2}, t\right)=W_{\bar{\eta}}\left(s_{1}, t\right) W_{\bar{\eta}}\left(s_{2}, t\right) W_{\bar{\eta}}^{*}\left(s_{3}, t\right)
$$

with

$$
\frac{1}{s_{1}}+\frac{1}{s_{2}}=\frac{1}{s_{3}}
$$

corresponding to addition of frequencies. This analysis tool was first introduced by Van Milligen et al. (1995) in a integrated form with respect to time, $\int_{T} B_{\bar{\eta}}\left(s_{1}, s_{2}, \tau\right) d \tau$, which was shown to measure the amount of phase coupling in the interval $T$ between wavelet components of scale lengths $s_{1}, s_{2}$ and $s_{3}$ or equivalently of frequencies $f_{1}, f_{2}$ and $f_{3}$. Here we set $s_{1}=s_{2}$, then $s_{3}=s_{1} / 2$ or $f_{1}=f_{2}=f_{3} / 2 . s_{1}$ is the corresponding scale for a frequency near peak frequency of the signal. Note that the bispectrum is a complex number, and its phase represents $\Theta_{B_{\bar{\eta}}}=\Theta_{1}+\Theta_{2}-\Theta_{3}$. In general, $\Theta_{B_{\bar{\eta}}}$ changes continuously between $-\pi$ to $\pi$. In the case of phase locking, however, it becomes constant or nearly so. Figure 4.13 shows $\Theta_{B_{\bar{\eta}}}$ corresponding to two frequencies near the peak frequency for the different packets. In P3, phase coupling starts near the break point and lasts for more than two periods. It is still considerable about $1.5 L_{c}$ downstream of the breaking point $\left(k_{c}\left(x-x_{b}\right)=10\right)$ which is consistent with figure 4.12. In the linear and incipient breaking cases, on the other hand, there is not such a strong phase coupling and $\Theta_{B_{\bar{\eta}}}$ continuously changes between $-\pi$ to $\pi$.

\subsection{Spanwise Averaged Velocity And Vorticity}

To study organized flow, which is approximated by the spanwise averaged flow field, evolution during and after breaking, P3 simulation is set to run for about 22 
periods after breaking. The signals have 44.1s length with $100 \mathrm{~Hz}$ sampling rate. Spanwise averaged velocity and vorticity are calculated using (2.35). DM measured the velocity field at multiple cross sections of the tank and found that the organized flow is two-dimensional to a good approximation. The numerical results also show that the organized flow is two-dimensional, with $\bar{v}$ an order of magnitude smaller than the spanwise averaged velocities in the other two directions. The spanwise averaged velocity field can be decomposed into

$$
\overline{\mathbf{u}}=\mathbf{u}_{w}+\mathbf{u}_{f w}+\mathbf{u}_{c}
$$

where $\mathbf{u}_{w}$ is the orbital velocities of the surface waves, $\mathbf{u}_{f w}$ is the velocities of the long forced waves induced by breaking and $\mathbf{u}_{c}$ is the current stemming from the momentum loss during the breaking and/or stokes drift. The mean current can be calculated by time averaging of the spanwise averaged velocity signal,

$$
\mathbf{u}_{c}=\overline{\overline{\mathbf{u}}}=\frac{1}{t_{2}^{*}-t_{1}^{*}} \int_{t_{1}^{*}}^{t_{2}^{*}} \overline{\mathbf{u}} d t^{*}
$$

where $t_{1}^{*}$ and $t_{2}^{*}$ cover the entire wave packet. During time integration for each grid point, when the point is above the free surface the velocity signal is zero. Figure 4.14 shows the spatial distribution of the normalized mean current and its horizontal averaged between $x^{*}=0.25$ and 1.25 , as well as the normalized horizontal averaged mass flux below the depth $z^{*}, \widehat{M^{*}}\left(z^{*}\right)=\int_{z_{1}^{*}}^{z^{*}} \widehat{u_{c}^{*}} d z^{*}$ where $z_{1}^{*}=-0.31$, for P3. The current induced by breaking is noticeable in $0.25<x^{*}<1.25$. The positive current penetrates more than a wave height with the magnitude on the order of $0.02 C_{c}$, up to $0.04 C_{c}$ if we perform time averaging in a shorter time span, near the still water depth. As shown by figure 4.14(c) a nearly constant return current at the lower depths compensates the positive current below the still water depth $\left.\widehat{\left(M^{*}\right.}\left(z^{*}=0\right) \approx 0\right)$. The mean current before and after breaking region is consistent with the effects of stokes drift in the dominant wave packet, reduced by the longer averaging time. 

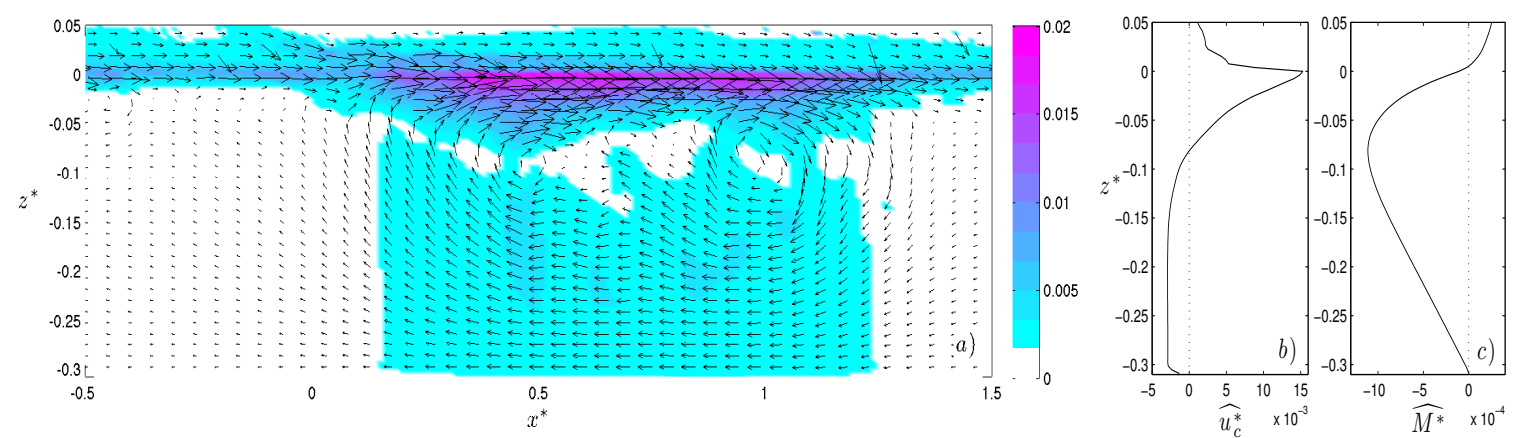

Figure 4.14: a) Spatial distribution of the normalized mean current, $\left.\mathbf{u}_{\mathbf{c}}^{*} ; b\right)$ normalized horizontal averaged mean current in the streamwise direction, $\widehat{u_{c}^{*}}$ and c) normalized accumulative horizontal averaged mass flux, $\widehat{M^{*}}$, in the breaking region for P3. Colors show $\sqrt{u_{c}^{* 2}+v_{c}^{* 2}}$.

To examine the time dependent distribution of the kinetic energy of the organized flow through different frequencies corresponding to $\mathbf{u}_{w}$ and $\mathbf{u}_{f w}$, wavelet analysis is performed. The modulus of the wavelet transform of the normalized spanwise averaged horizontal velocity signal after subtracting its mean, $\overline{u^{*}}-u_{c}^{*}$, is shown in figure 4.15. The frequency band of the input packet, $0.635<f / f_{c}<1.365$, is shown by the dotted lines. As we can see the main part of the energy is carried by the surface waves and distributed across this band. After $t^{*} \sim 4$, when the packet passes the breaking region, the energy in this band decreases to a negligible amount at $t^{*} \sim 8$, while the energy in the low frequency long forced waves remain noticeable and similar at the lower depths. To remove the repeatable surface wave velocities from $\overline{u^{*}}-u_{c}^{*}$ time series, we perform low-pass filtering with a cut-off frequency $f_{c u t} / f_{c}=0.34$ (dashed line in figure 4.15) using the overlap-add method as in RM. The vorticity of the spanwise averaged flow is calculated based on the raw spanwise averaged signals. Figure 4.16 shows snapshots of spatial distribution of the low-pass filter spanwise averaged velocity field and vorticity of the organized flow, $\overline{\omega_{y}}$. It clearly shows the formation of the forced long waves in the breaking region and the large coherent vortices which propagate slowly downstream and deepen. The dominant positive $\overline{\omega_{y}}$ indicates the clockwise rotation of the vortices. At $t^{*}=15$ the large vortex between $0.25<x^{*}<1.25$ is very clear, while 

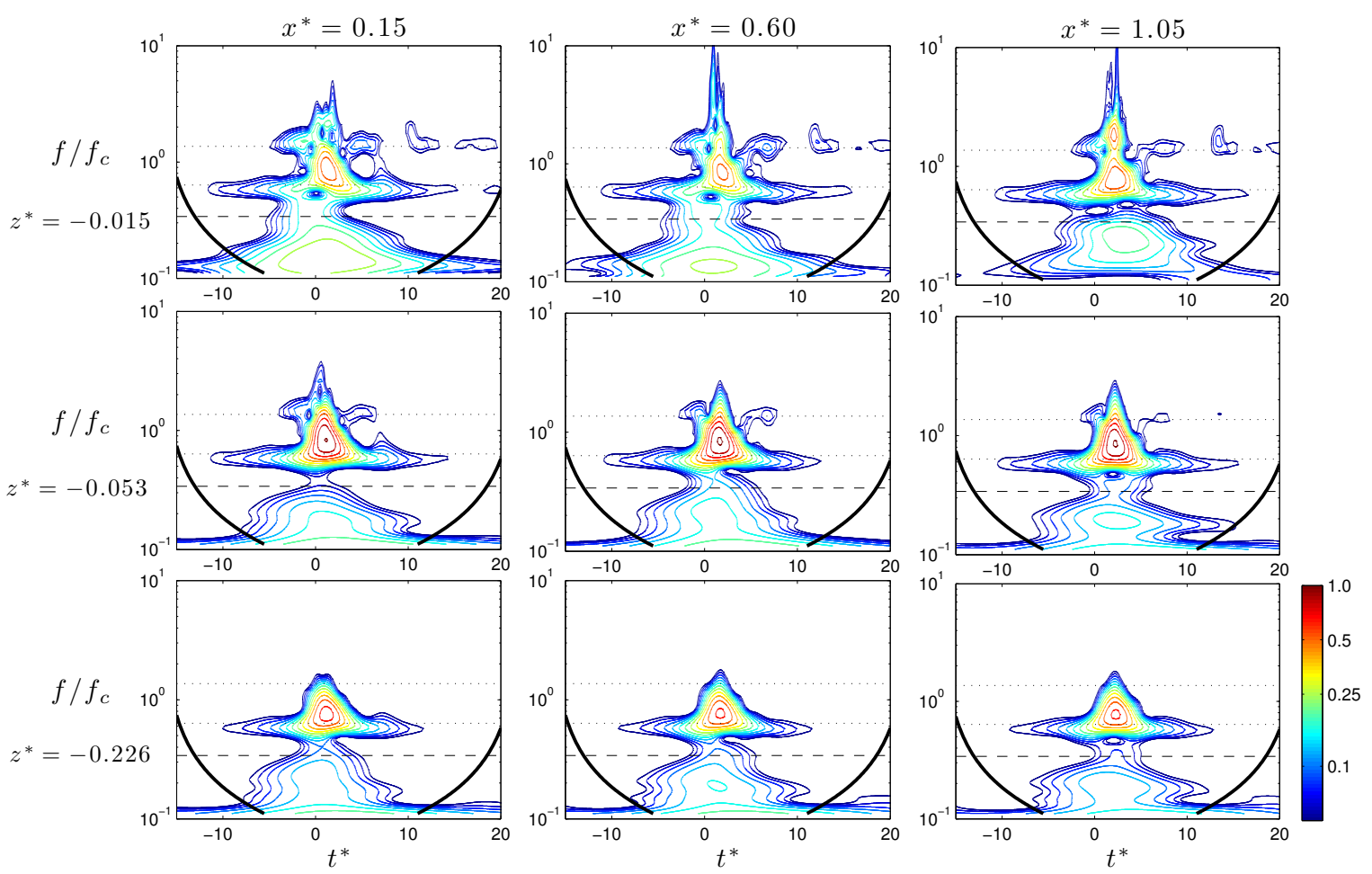

Figure 4.15: Modulus of the wavelet transform of $\overline{u^{*}}-u_{c}^{*}$ for P3. (left) $x^{*}=0.3$; (middle) $x^{*}=0.6$ and (right) $x^{*}=1.05$. The elevation of corresponding signal location in each row is the same and is shown at the left column. Dotted lines indicate the frequency band of the input packet. Dashed line shows the cut-off frequency for low-pass filtering. Regions below the solid lines on either end indicate the ?cone of influence,? where edge effects become important.

the velocities before and after it are nearly zero. The strong flow near the surface with the nearly constant return flow at lower depths form the large coherent vortex, while multiple smaller vortices can be recognized at the beginning and the end of the large coherent vortex. As time proceeds, the small vortices grow, detach from the surface and propagate very slowly downstream. Based on figure 4.16, their averaged horizontal and vertical speed can be estimated as $\approx 0.01 C_{c}$ and $0.001 \sim 0.003 C_{c}$, respectively. The large coherent breaking-induced vortex is also observed by RM and DM.

RM measured the velocity field using LDV at seven elevations and seven $x$ locations in the breaking region. They performed filtering both on the ensemble averaged 

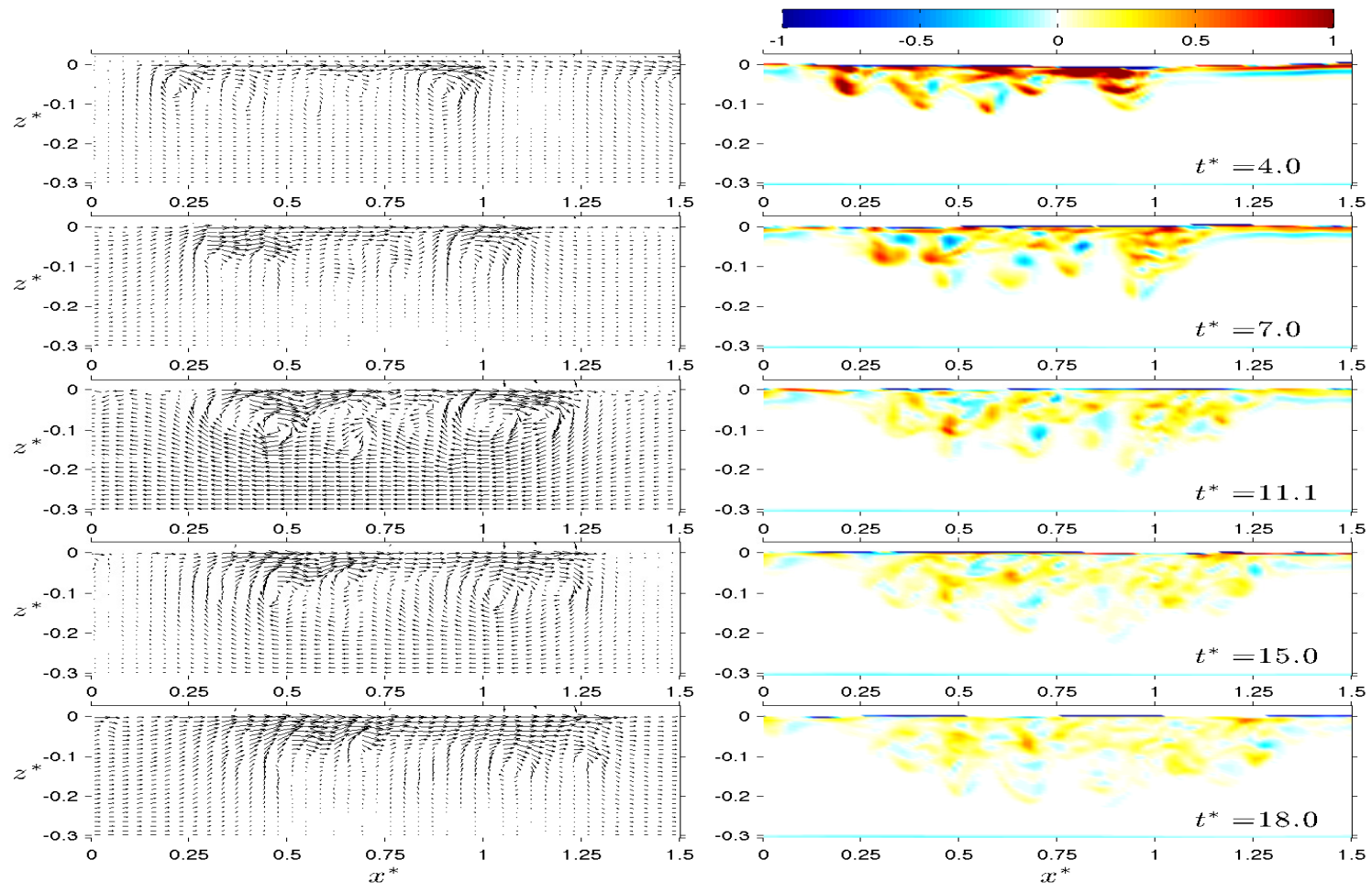

Figure 4.16: (left) Normalized low-pass filtered spanwise averaged velocity field, $\left(\overline{u^{*}}, \overline{v^{*}}\right)$ and (right) normalized vorticity of the organized flow, $\overline{\omega_{y}^{*}}$, for P3. The arrays are normalized in each figure and the color shows the intensity
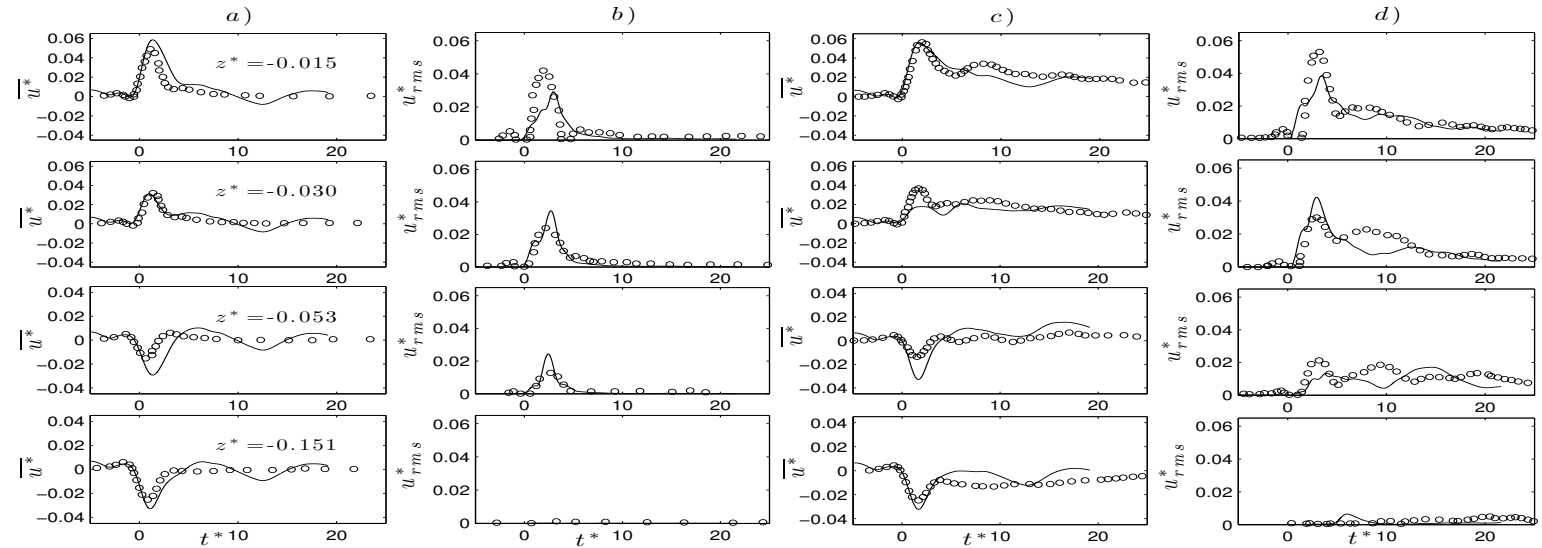

Figure 4.17: Normalized low-pass filtered spanwise averaged and r.m.s. velocities for P3 at $(a-b) x^{*}=0.15$ and $(c-d) x^{*}=0.60$ in different elevations. Circles are the measurements of the corresponding case adopted from RM figure 42 and figure 46. 
(based on the 10 repeats of the packet) and r.m.s. velocity in the same manner. For r.m.s velocity the filtering was first performed on the variance of the turbulent velocity and then square root was taken. Figure 4.17 shows the model results vs measurements for P3 at $x^{*}=0.15$ and $x^{*}=0.60$ from very close to the free surface to $z^{*}=-0.151$ $(\approx z=-d / 2)$. Although we do not expect exact correlations for a point-wise comparison in the breaking region especially in this non stationary event, the results show that the model captures the spatial and temporal evolution of the organized and turbulent velocity in the breaking region reasonably well. At $x^{*}=0.15$ the slow variation of the r.m.s and spanwise averaged signals clearly shows the passage of the TKE cloud and the coherent vortices induced by the breaking. Further downstream at $x^{*}=0.60$, the r.m.s. turbulent velocity initially has the same increase as the TKE cloud arrives, but it only decreases to $0.01 C_{c}$ at $t^{*}=20$. Based on the experiments it remains about $0.005 C_{c}$ even up to $t^{*}=60$. In addition, we can conclude that the spanwise averaging from (2.35) and (2.36) are a good approximation for the ensemble averaging in this problem.

\subsection{Turbulent Velocity And Vorticity}

The r.m.s. of turbulent fluctuations is calculated using (2.36). Figure 4.18 shows the time dependent spatial distribution of the r.m.s turbulent velocities and vorticities in the breaking region for $\mathrm{P} 1$. During the active breaking period, $0<t^{*}<1$, the turbulent motions have very high intensity and are concentrated near the free surface. As time proceeds, they become more uniform and are mixed down more than two wave heights. At the initial stage of breaking, turbulent fluctuations in the streamwise direction, $u_{r m s}$, are much bigger than in the other two directions, with a maximum of $0.3 C_{c}$, and in most of the region $w_{r m s} \approx v_{r m s}$. During the first jet impact and splash cycles the r.m.s. of turbulent vorticity in the spanwise direction, $\omega_{y, r m s}$, is bigger than other two components, especially in the forward face of the wave. At the backside, instead, $\omega_{y, r m s}<\omega_{x, r m s} \approx \omega_{z, r m s}$. If we refer to the flow field at $t^{*} \geq 4$ as a background level, figure 4.18 shows that the r.m.s of turbulent velocity and vorticity at 
the background level initially are an order of magnitude smaller than that during active breaking. In addition, both the velocity and vorticity field become more isotropic, and the turbulent cloud is well mixed in the three directions.

Existence of a highly anisotropic surface layer is one of the characteristic features of free-surface turbulence, regardless of flow type (Shen \& Yue 2001, Lin et al. 2008). In the absence of surface waves, the shear stresses vanish near the free surface and vertical component of the turbulent velocity decreases considerably as the free surface is approached. This creates a thin surface layer where the velocity derivatives are highly anisotropic. The blockage effects of the surface causes anisotropy of the velocity components themselves over a thicker region (Shen \& Yue 2001). Under surface waves, on the other hand, the orbital motions strongly enhance the turbulent fluctuations, especially the vertical component (Lin et al. 2008, figure 16). To examine the turbulence structure in the vertical direction, we performed time dependent horizontal averaging on lines parallel to the instantaneous spanwise averaged free-surface locations. Because the process is non-stationary and non-homogeneous, especially during the active breaking period, this averaging method only gives a general trend of vertical variation of the turbulence structure. Figures 4.19 and 4.20 show the horizontally averaged, (), turbulent velocity and vorticity variance for $\mathrm{P} 1$ and $\mathrm{SP} 1$, respectively. At $t^{*}=0.5$ where surface waves are strong, $\widehat{\overline{u^{\prime 2}}} \gg \widehat{\overline{v^{\prime 2}}} \approx \widehat{\overline{w^{\prime 2}}}$. As time proceeds and surface waves pass the breaking region, $\widehat{w^{\prime 2}}$ starts to decrease near the free surface and $\widehat{w^{\prime 2}}$ is much smaller than $\widehat{\overline{v^{\prime 2}}}$, while at the lower depths, $\widehat{w^{\prime 2}}$ becomes on the order of or larger than $\widehat{\overline{v^{\prime 2}}}$. Vertical variation of the vorticity components generally have a larger gradient. In a very thin layer near the free surface, $\widehat{\overline{\omega_{y}^{\prime 2}}}$ is bigger than the other two components during the active breaking. In $\mathrm{P} 1$, the anisotropy reduces as time proceeds, and horizontal averaged turbulent vorticity variance in the streamwise direction, $\widehat{\omega_{x}^{\prime 2}}$, becomes comparable and even larger than the other two components. In SP1, on the other hands, the surface layer is more anisotropic than the plunging case with the anisotropy both in the turbulent velocity and vorticity field remains noticeable after active breaking. 

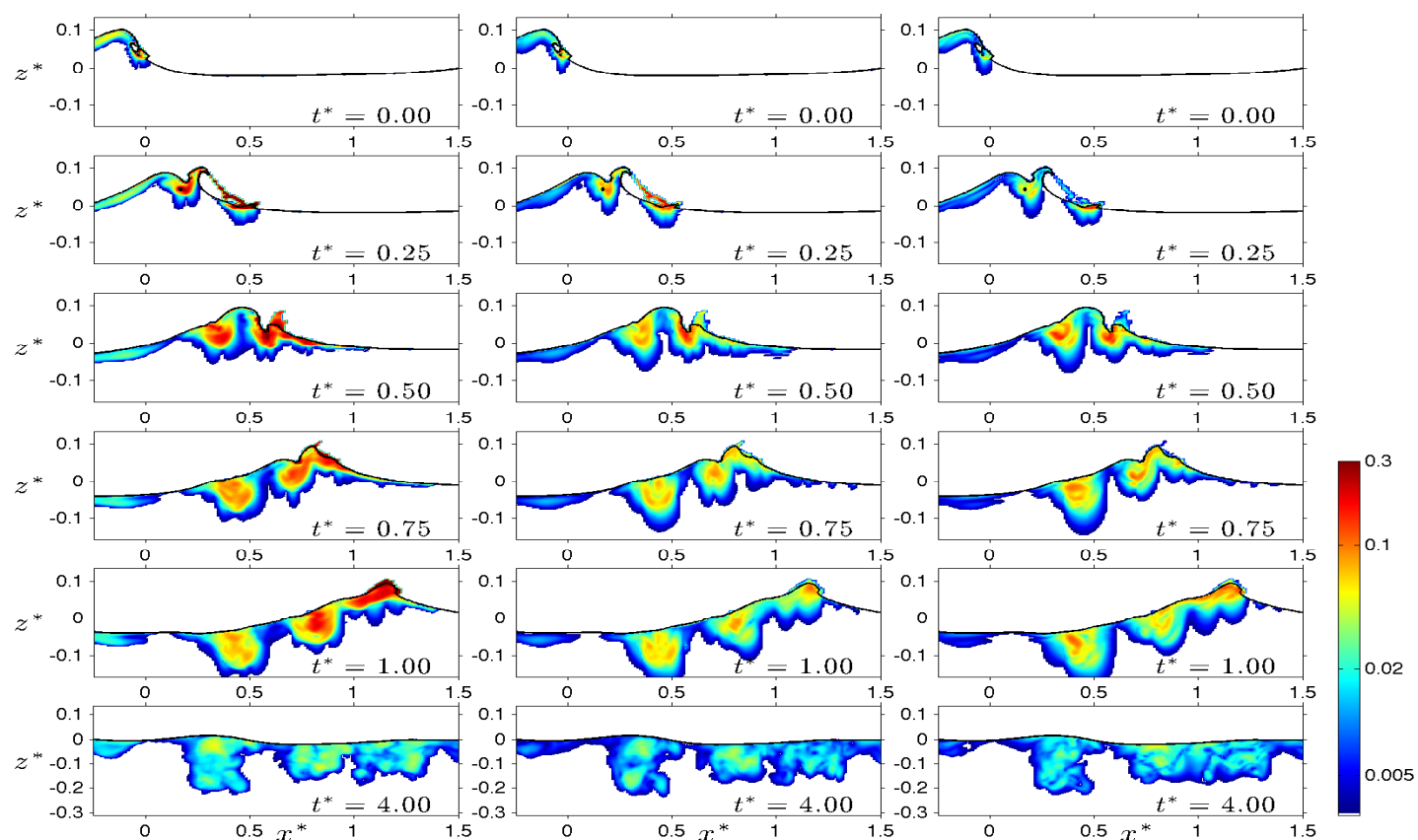

(a) r.m.s. of turbulent velocity components (left) $u_{r m s}^{*}$; (middle) $w_{r m s}^{*}$ and (right) $v_{r m s}^{*}$
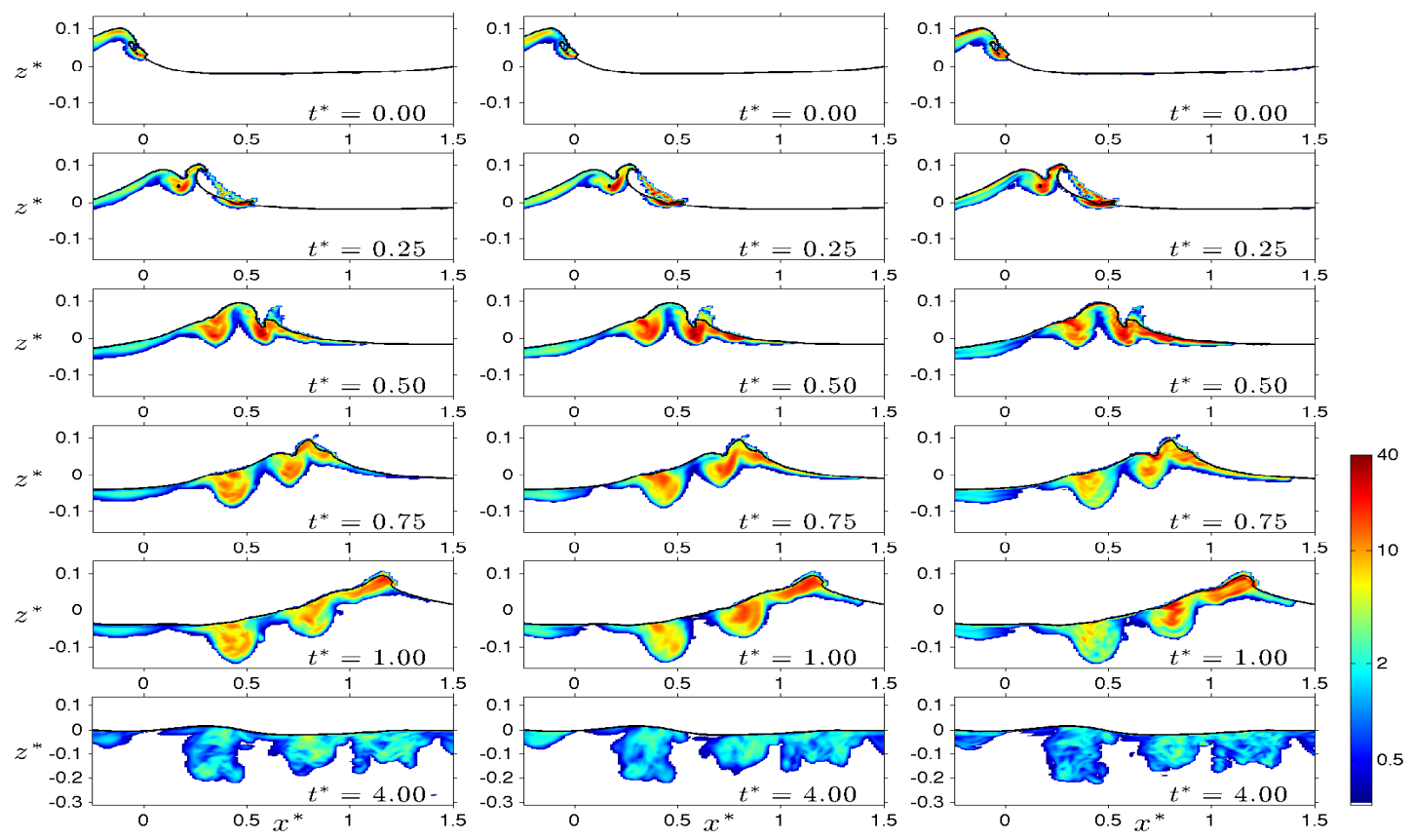

(b) (left) $\omega_{x, r m s}^{*} ;\left(\right.$ middle) $\omega_{z, r m s}^{*}$ and (right) $\omega_{y, r m s}^{*}$

Figure 4.18: Snapshots of turbulent r.m.s. of a) velocities and b) vorticity components for P1. 

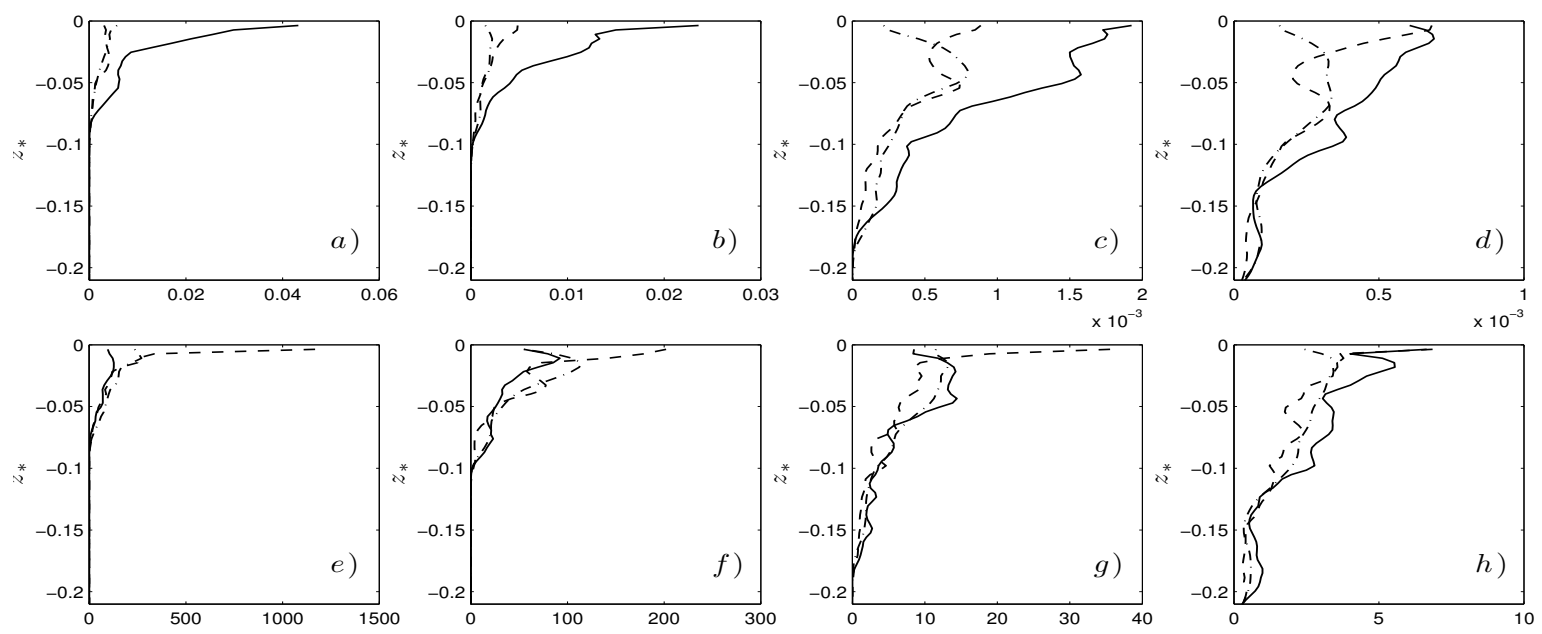

Figure 4.19: Horizontally averaged turbulent velocity and vorticity variance for P1. $(a-d)-\widehat{\widehat{u^{\prime 2}}} ;-\cdot-\widehat{\widehat{w^{\prime 2}}} ;---\widehat{\overline{v^{\prime 2}}}$ and $(e-h)-\widehat{\widehat{\omega_{x}^{\prime 2}}}$; $-\cdot \widehat{\overline{\omega_{z}^{\prime 2}}} ;--\widehat{\widehat{\omega_{y}^{\prime 2}}} \cdot(a, e) t^{*}=0.5 ;(b, f) t^{*}=1.0 ;(c, g) t^{*}=2.0$ and $(d, h) t^{*}=4.0$.
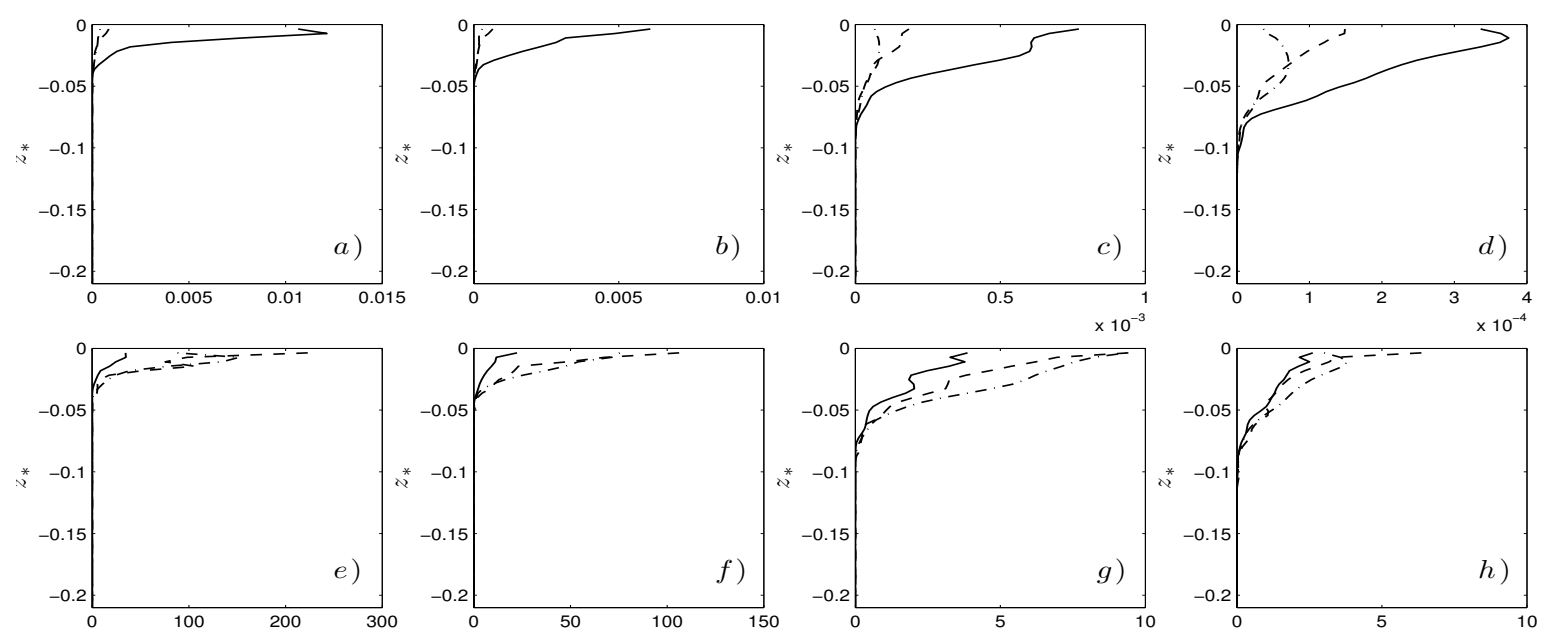

Figure 4.20: Horizontally averaged turbulent velocity and vorticity variance for SP1. $\quad(a-d)-\widehat{\widehat{u^{\prime 2}}} ;-\cdot-\widehat{\widehat{w^{\prime 2}}} ;---\widehat{\widehat{v^{\prime 2}}}$ and $(e-h)-\widehat{\widehat{\omega_{x}^{\prime 2}}}$; $-\cdot \widehat{\widehat{\omega_{z}^{\prime 2}}} ;--\widehat{\widehat{\omega_{y}^{\prime 2}}} \cdot(a, e) \quad t^{*}=0.5 ;(b, f) t^{*}=1.0 ;(c, g) t^{*}=2.0$ and $(d, h) t^{*}=4.0$. 

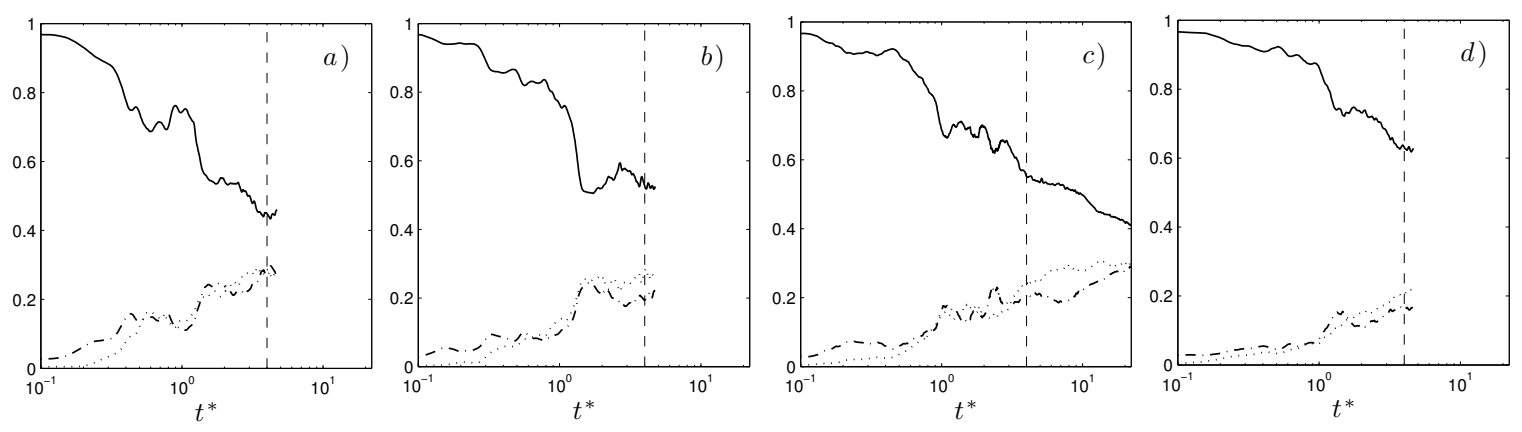

Figure 4.21: Ratios of the different turbulent fluctuation components to total TKE, $\check{\bar{k}}$, for a) P1; b) P2; c) P3 and d) SP1. - $\overline{u^{\prime 2}} / 2 \check{\bar{k}} ;-\cdot-\check{\overline{w^{\prime 2}}} / 2 \overline{\bar{k}}$; ...... $\overline{v^{\prime 2}} / 2 \dot{\bar{k}}$.

Furthur, we integrate $\overline{u_{i}^{\prime 2}}$ over the entire breaking region

$$
\check{\overline{u_{i}^{\prime 2}}}=\int_{x_{1}^{*}}^{x_{2}^{*}} \int_{z_{1}^{*}}^{\eta^{*}} \overline{u_{i}^{\prime 2}} d z^{*} d x^{*}
$$

where $z_{1}^{*}=-0.31, x_{1}^{*}=-0.25$ and $x_{2}^{*}=2.0$ cover the region. Then we can calculate contribution of each turbulent velocity component to total TKE in the breaking region, $\check{\bar{k}}$. Figure 4.21 shows these ratios for the different breakers. During active breaking, $\check{u^{\prime 2}}$ accounts for most of $\check{\bar{k}}$, and $\check{\overline{w^{\prime 2}}}>\check{\overline{v^{\prime 2}}}$. But, as the TKE cloud evolves, the $\check{\overline{u^{\prime 2}}}$ ratio continuously decreases while the other two increase, and $\check{w^{\prime 2}}<\check{v^{\prime 2}}$.

Similarly, we integrate variance of the turbulent vorticity components. Figure 4.22 demonstrates their contributions to total mean square turbulent vorticity, $\check{\omega_{i}^{\prime 2}}$, where $\omega_{i}^{\prime 2}=2\left(\Omega-\Omega^{m}\right), \Omega=\frac{1}{2} \omega_{i} \omega_{i}$ is enstrophy and $\Omega^{m}=\frac{1}{2} \bar{\omega}_{i} \bar{\omega}_{i}$ is one half of the squared mean vorticity. Initially $\check{\bar{\omega}_{y}^{\prime 2}}$ is the dominant component, which means that the event is mainly 2D. It decreases fairly rapidly while the streamwise and vertical components increase because of the shift of the two-dimensional vortex structures to three-dimensional. After the active breaking period, all three components become comparable. At later times $t^{*} \gg 1$, the streamwise component ratio continuously increase and the other two components remain at the same order. 

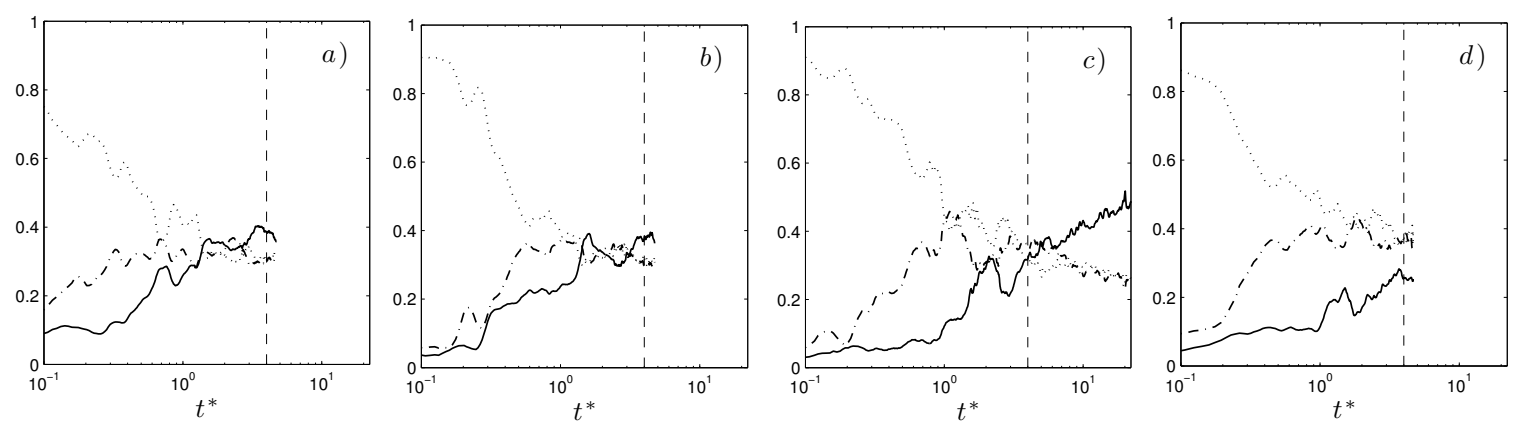

Figure 4.22: Ratios of the different turbulent vorticity components to mean square turbulent vorticity, $\check{\omega^{\prime 2}}$, for a) P1; b) P2; c) P3 and d) SP1. $-\frac{\check{\omega^{\prime 2}}}{x} / \check{\omega^{\prime 2}}$; $-\cdot-\check{\omega_{z}^{\prime 2}} / \widetilde{\omega_{i}^{\prime 2}} ; \quad \cdots \cdots \overline{\omega_{y}^{2}} / \check{\omega_{i}^{\prime 2}}$.

\subsection{TKE And Enstrophy}

Figures 4.23 and 4.24 show the normalized spanwise averaged TKE and enstrophy for P1, SP1 and P3, respectively. The spatial distribution and time evolution of both quantities are very similar. TKE cloud evolution in P3 is very comparable to the measured turbulent intensities by RM. In addition, TKE cloud boundary is consistent with the digitized dye trace shown in RM figure 47 which indicates model can fairly well capture the transition between non-turbulent and high turbulent regions. The background level of TKE and enstrophy intensities initially, $t^{*} \approx 4$, are two order of magnitude smaller than that during the active breaking period. As expected, in the background flow, e.g. between $4.0<t^{*}<22.0$ in P3, the decay rate of TKE and enstrophy becomes much smaller.

The penetration of the TKE and enstrophy seem to be well correlated with the wave height for both plunging and spilling breakers. For all breakers, the mixed layer depth is approximately $0.5 \mathrm{H}$ for the spilling and $0.75 \mathrm{H}$ for the plunging breakers during the active breaking period, where $H$ is the wave height before breaking. It becomes about twice deeper at $t^{*}=4.0$, with $1 H$ for the spilling and $1.5 H$ for the plunging breakers. In $\mathrm{P} 1$, the mixed layer depth further increases to about $2.5 \mathrm{H}$ at $t^{*}=11.0$. At later times, the averaged layer depth remains nearly constant with only local obliquely deepening. This is consistent with the measurement of RM and DM 

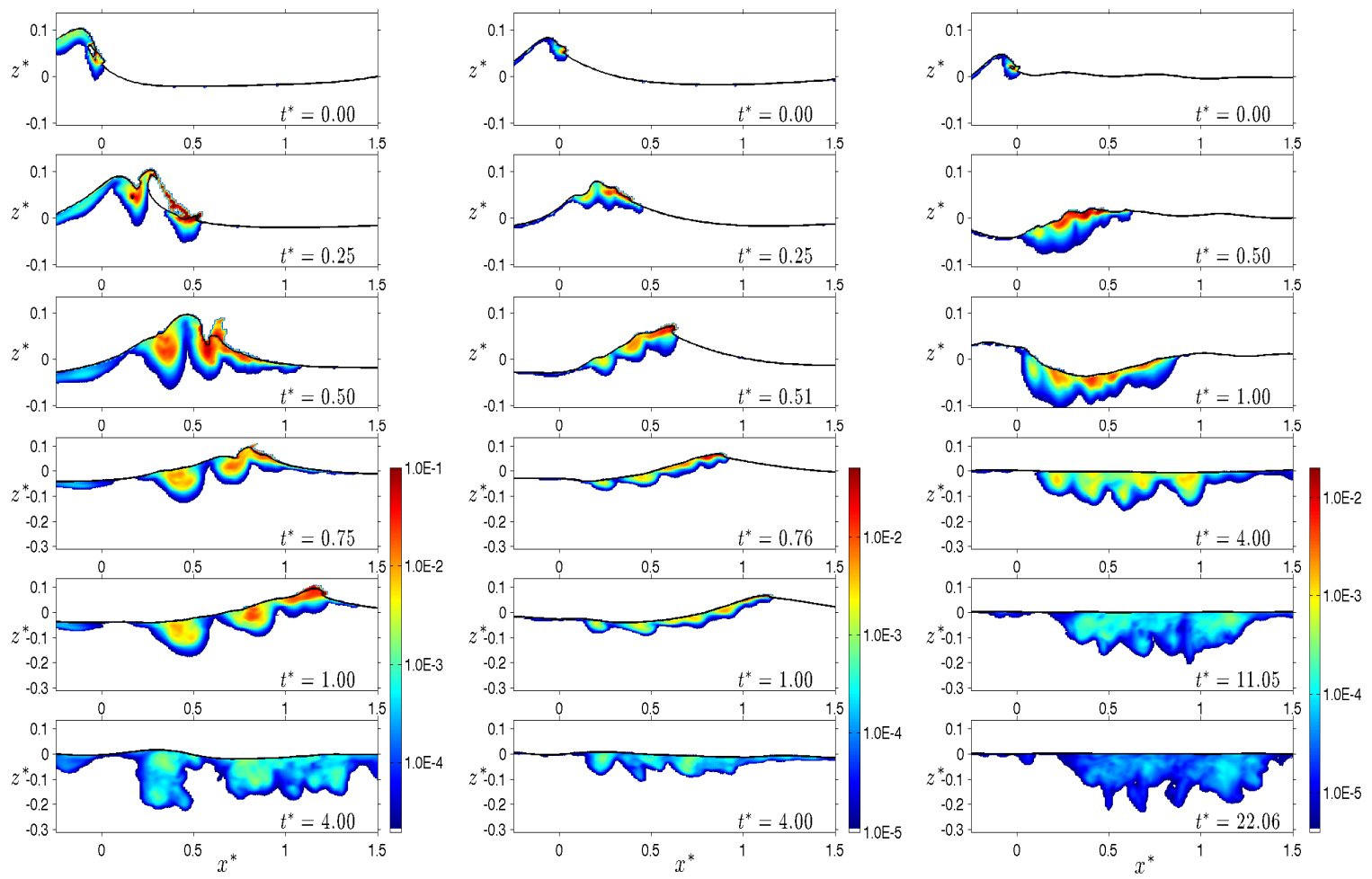

Figure 4.23: Snapshots of the normalized spanwise averaged TKE, $\bar{k}$, for (left) P1; (middle) SP1 and (right) P3. The reference value is $C_{c}^{2}$.

in which the TKE cloud thickness became nearly constant from $t^{*} \sim 10$ up to $t^{*} \sim 50$.

Figures 4.24 and 4.4 demonstrate that the regions with high enstrophy are very well correlated to the high void fraction regions of the bubble plume. This is because of the transport of dispersed bubbles by the vortex structures. In P1, because of a strong jet impact, there is a clear downburst of turbulence and vorticity in $0.25<x^{*}<0.5$, which leads to formation of a separate turbulent region transported deeper than the other regions. The following splash cycle also generates a weaker downburst. These two downburst type vortices are responsible for forming two semicircular bubble clouds, observed also by Rojas \& Loewen (2010). In P3, the jet-impact is weaker and the resulting turbulent cloud is more uniform in the streamwise direction with the obliquely descending extension of enstrophy and TKE. 

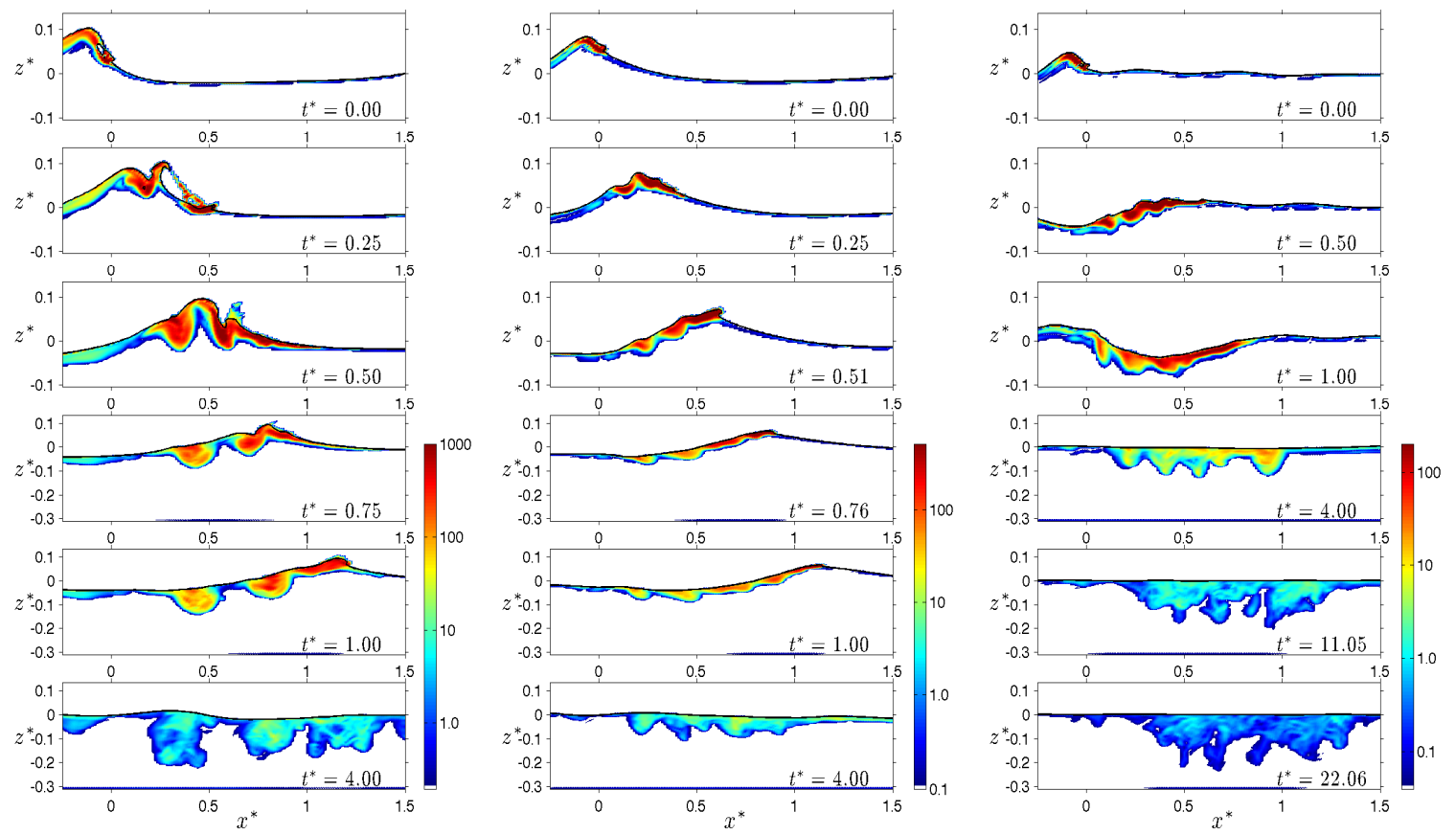

Figure 4.24: Snapshots of the normalized spanwise averaged enstrophy, $\bar{\Omega}$, for (left) $\mathrm{P} 1$; (middle) SP1 and (right) P3. The reference value is $T_{c}^{-2}$.

Figure 4.25 shows the time evolution of total TKE and enstrophy in the breaking region for the different breakers. The integrated TKE of the corresponding case in $\mathbf{R M}$ is shown in figure 4.25a. $\mathbf{R M}$ estimated the ensemble averaged volume integrated squared turbulent components, $u^{\prime 2}, w^{\prime 2}$, based on a pointwise measurements in a relatively coarse grid. After $t^{*}=4$, the model results are fairly consistent with the measurements at which TKE cloud is well mixed, and integration based on a coarse grid which is used in the measurement, would be a good approximation. Figures 4.25b and $4.25 \mathrm{c}$ show the time evolution of the total TKE, $\check{\bar{k}}$, and total enstrophy, $\check{\bar{\Omega}}$, in the breaking region for the other cases. The decay rate of both $\check{\bar{k}}$ and $\check{\bar{\Omega}}$ increase with increasing breaking intensity. After active breaking, $\check{\bar{k}}$ decays in the form of $\left(t^{*}\right)^{-n}$ in which $n$ varies from 0.5 to 1.0 for spilling to plunging breaking. $\check{\Omega}$ has a similar decay form with larger $n$ varies from 1.0 to 1.5 for spilling to plunging breaking. RM and $\mathbf{D M}$ also found $\left(t^{*}\right)^{-n}$ dependence with $n \approx 1.0$, for total TKE in the breaking 

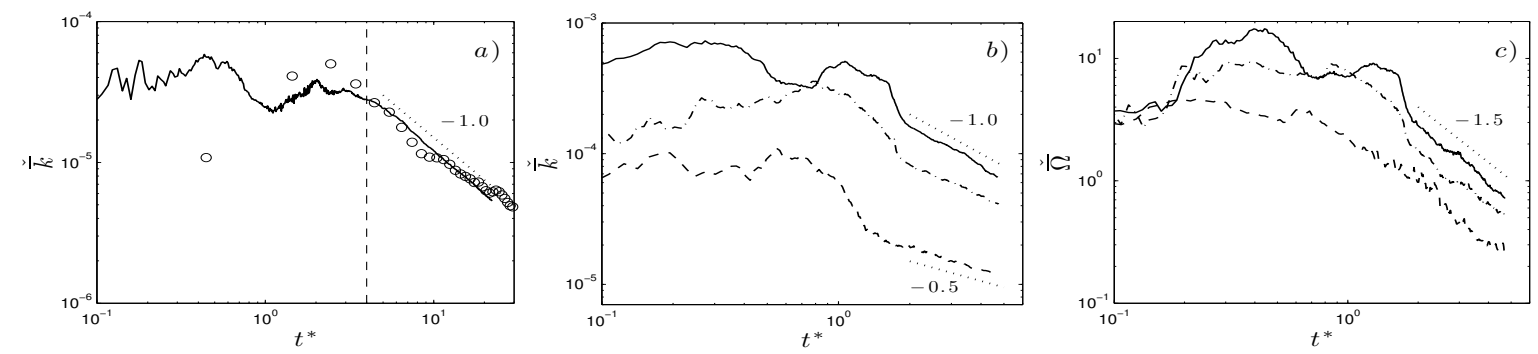

Figure 4.25: a) Total TKE, $\check{\bar{k}}$ for P3; $b$ ) total TKE, $\check{\bar{k}}$ and $c$ ) total enstrophy $\check{\bar{\Omega}}$ for $\mathrm{P} 1,-\cdot-\mathrm{P} 2$ and $---\mathrm{SP} 1$. Dotted lines indicate $\left(t^{*}\right)^{n}$ where $n$ is the number on the lines. Circles are $\sum_{i=1}^{7} \Sigma_{j=1}^{7} \frac{1}{2}\left(<u^{\prime 2}(i, j)>+2<\right.$ $\left.w^{\prime 2}(i, j)>\right) A(i, j)$ adopted from $\mathbf{R M}$ figure 54. The reference values for TKE and enstrophy are $L_{c}^{2} C_{c}^{2}$ and $L_{c}^{2} T_{c}^{-2}$, respectively.
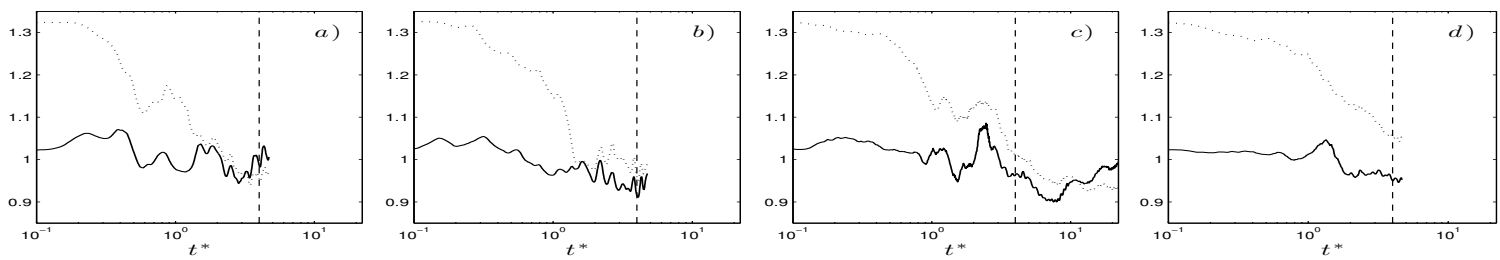

Figure 4.26: The two different estimates of three-dimensional TKE for a) P1; b) P2; c) P3 and d) SP1. - $\check{\bar{k}}^{1} / \check{\bar{k}}^{2} ; \ldots . . \check{\bar{k}}^{1} / \overline{\bar{k}}$.

region based on the 2D measurements of the streamwise and vertical turbulent velocity components.

In $2 \mathrm{D}$ numerical simulations and most of the experimental measurements, the out of plane velocity component, $v$, is missing. To account for this missing component in TKE calculation, Svendsen (1987) argued there are similar characteristics between the turbulent components under quasi-periodic surf zone breaking waves and plane wakes. He estimated ensemble averaged three-dimensional TKE, $\bar{k}=\frac{1}{2} \overline{u_{i}^{\prime 2}}$, as

$$
\bar{k} \approx \bar{k}^{1}=\frac{1.33}{2}\left(\overline{u^{\prime 2}}+\overline{w^{\prime 2}}\right) .
$$

Based on figure 4.21, we may estimated $k$ as

$$
\bar{k} \approx \bar{k}^{2}=\frac{1}{2}\left(\overline{u^{\prime 2}}+2 \overline{w^{\prime 2}}\right) .
$$



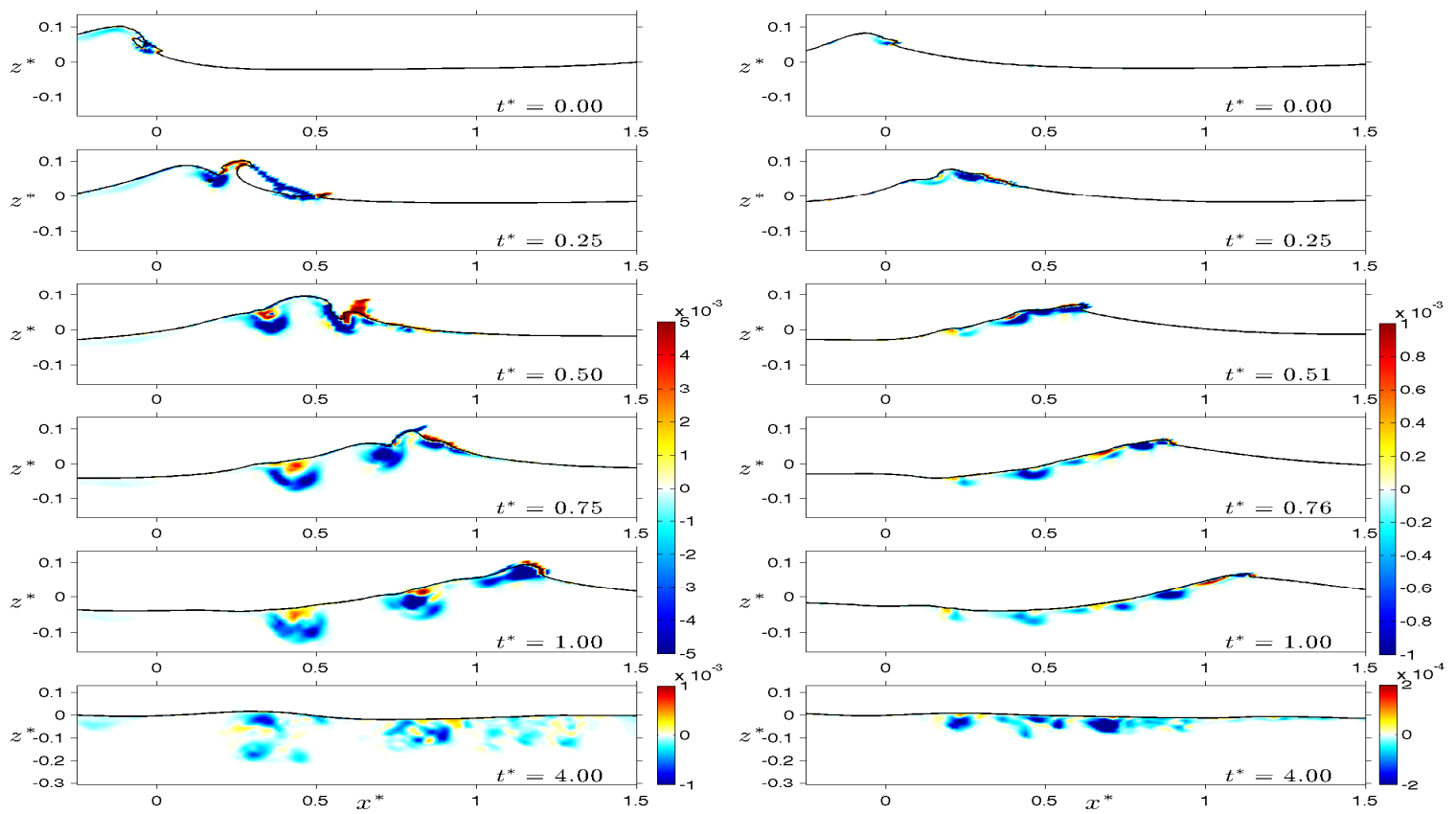

Figure 4.27: Spanwise averaged Reynold stress in longitudinal plane, $\overline{u^{\prime} w^{\prime}} / C_{c}^{2}$ for (left) P1 and (right) SP1.

Figure 4.26 shows $\check{\bar{k}}^{1} / \check{\bar{k}}$ and $\check{\bar{k}}^{2} / \check{\bar{k}}$. $\check{\bar{k}}^{1}$ overestimates $\check{\bar{k}}$ about $30 \%$ while $\check{\bar{k}}^{2}$ gives a better results especially during the active breaking period, with the maximum error less than $10 \%$. Both estimates give reasonable results for $t^{*}>1$.

\subsection{Reynolds Stress}

The Reynolds stresses transfer momentum from the organized surface wave field into the bulk of the fluid. Figure 4.27 shows $\overline{u^{\prime} w^{\prime}}$ for $\mathrm{P} 1$ and $\mathrm{SP} 1 . \overline{u^{\prime} w^{\prime}}$ is dominantly negative, consistent with the mechanism through which currents are generated by breaking due to the vertical flux of the positive horizontal momentum lost from the surface waves.

\subsection{Energy Dissipation}

Energy dissipation is one of the least understood components of the near-surface dynamics of the ocean. The breaking induced dissipation rate is much greater than the 
other sources of dissipation especially during active breaking. In addition, breaking waves are highly intermittent both in time and space. Thus, the mixing and energy dissipation at the near-surface layer are highly intermittent and dominant by the individual breaking events, with the background levels of turbulence and dissipation rate being considerably lower Melville (1994).

Based on scaling arguments, Duncan $(1981,1983)$ showed that the wave dissipation per unit length of breaking crest can be written in the form of

$$
\check{\epsilon}_{t o t a l}=b \rho g^{-1} c^{5}
$$

where $g$ is the gravitational acceleration, $c$ is the phase speed of the wave and $b$ is a breaking parameter. Phillips (1985) defined a distribution $\Lambda(c)$ so that $\Lambda(c) d c$ represents the average total length of breaking fronts per unit surface area traveling with velocities in the range $(c, c+d c)$. He then formulated the average rate of energy loss for breaking waves with speeds in the range $(c, c+d c)$ per unit surface area as

$$
\check{\epsilon}_{\text {total }}(c) d c=b \rho g^{-1} c^{5} \Lambda(c) d c
$$

This concept is very interesting especially for the large scale models with relatively large time scales, e.g. wind-wave models in which a whole breaking event is SGS and needs to be parameterized based on the spectral characteristics. However, as summarized by Drazen et al. (2008), the available literature shows a large scatter for $b$. In addition, so far only a few published $\Lambda(c)$ distributions exist, and it may need to be calibrated based on the specific area of the interest.

In this section, we first examine the bubble- and shear induced dissipation and their contribution to the total dissipation under a breaking event - note that the former, is commonly ignored in 3D LES simulations of breaking waves. Then, we discuss time dependent behavior of the breaking parameter, $b$. In addition, the time averaged dissipation rate per unit length of breaking crest is obtained. 

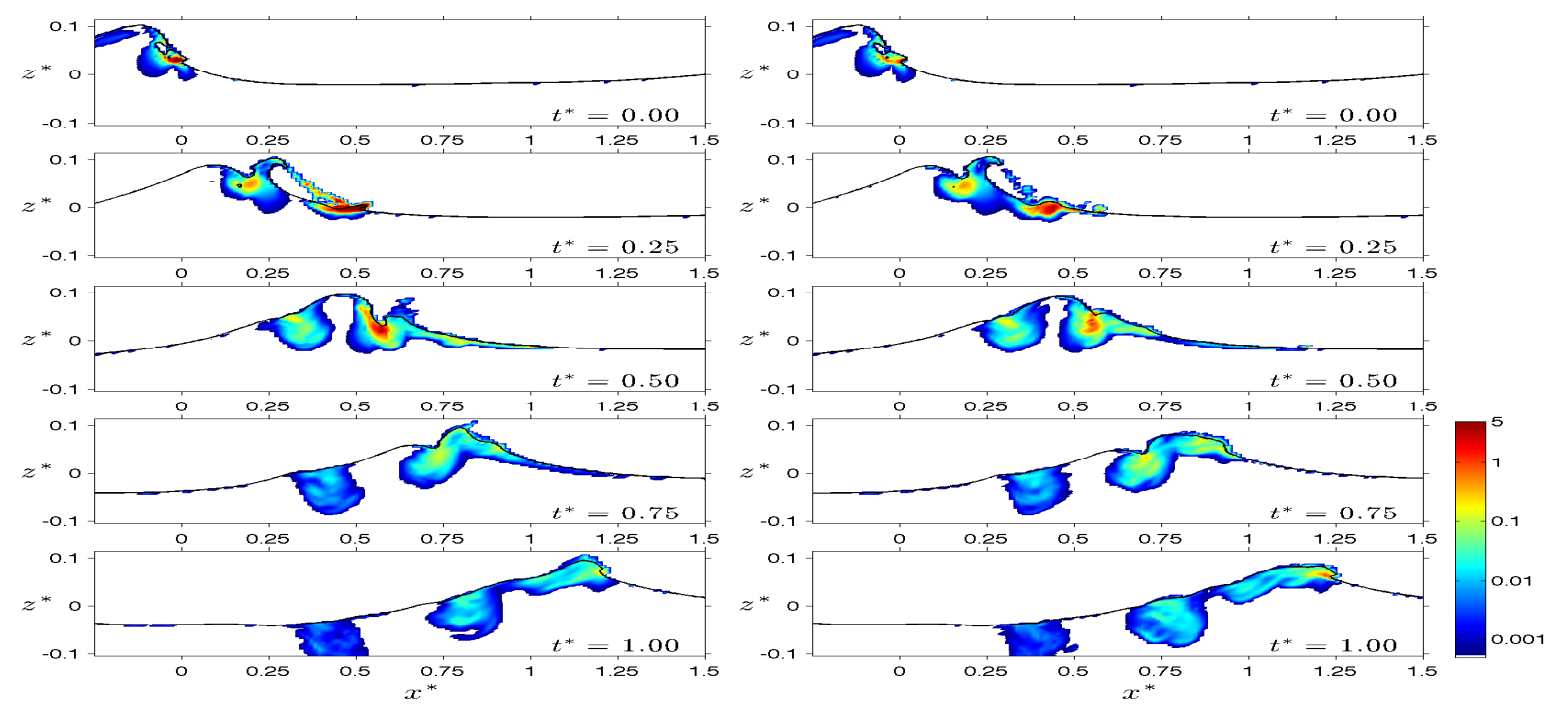

Figure 4.28: Snapshots of the spanwise averaged SGS dissipation rate, $\overline{\varepsilon_{s g s}}\left[m^{2} s^{-3}\right]$ for P1, from the simulation (left) with and (right) without the inclusion of the dispersed bubbles.

\subsubsection{Shear- and bubble-induced dissipation}

Dissipation rate of the resolved kinetic energy per unit mass is

$$
\varepsilon_{\text {total }}=\varepsilon_{r}+\varepsilon_{\text {sgs }}
$$

where $\varepsilon_{r}=2 \nu \mathcal{S}_{i j} \mathcal{S}_{i j}=\nu|\mathcal{S}|^{2}$ is viscous dissipation rate directly from the resolved velocity field and $\varepsilon_{\text {sgs }}$ represents the rate of transfer of energy from the resolved motions to the SGS motions. In the present study, $\varepsilon_{s g s}=-\tau_{i j}^{d} \mathcal{S}_{i j}=\varepsilon_{S I}+\varepsilon_{B I}$ consists of two different dissipation mechanisms. $\varepsilon_{S I}=\nu_{S I}|\mathcal{S}|^{2}$ is a conventional shear-induced dissipation rate similar to a single phase turbulence. The additional term, $\varepsilon_{B I}=$ $\nu_{B I}|\mathcal{S}|^{2}$, accounts for turbulent SGS motions generated by the dispersed bubbles and enhances the dissipation rate. At high Reynolds number, with the filter width much larger than the Kolmogorov length scale (typical in LES), $\varepsilon_{r}$ is usually negligibly small, and if the model resolves most of TKE, we can approximate the total dissipation rate, $\varepsilon$, by $\overline{\varepsilon_{s g s}}$. This is equivalent to assuming, there is a close balance between production and dissipation in the averaged kinetic energy of SGS motions (Pope 2000). 
Figure 4.28 shows snapshots of the spatial distribution of $\overline{\varepsilon_{s g s}}$ for $\mathrm{P} 1$ with and without the inclusion of dispersed bubble phase. The regions with a high dissipation rate are collocated with the high vorticity and TKE regions. $\overline{\varepsilon_{s g s}}$ is very large, $O\left(1 m^{2} s^{-3}\right)$, at the jet/splash impact and the bore front regions. The corresponding Hinze scale of bubble diameter then can be estimated as $a_{H} \cong c\left(\frac{\gamma}{\rho}\right)^{3 / 5} \varepsilon^{-2 / 5} \approx 1 \mathrm{~mm}$, where $c=0.36 \sim 0.5$ and $\frac{\gamma}{\rho}=7.3 \times 10^{-5}$ (see Garrett et al. 2000). This is consistent with our choice of $a_{H}=1 \mathrm{~mm}$, for the bubble size distribution. The local values of dissipation rate decrease very quickly. At the end of active breaking, they become at least two orders of magnitude smaller. These regions have a relatively high void fraction, and comparing the results of the simulation with and without bubble phase clearly shows enhancement of the dissipation rate in these bubbly regions. Figure 4.29 shows the integrated viscous as well as shear- and bubble-induced SGS dissipation rate in the breaking region for $\mathrm{P} 1, \mathrm{P} 2, \mathrm{SP} 1$ and $\mathrm{S} 1$. The bubble-induced dissipation is very noticeable during the jet/splash cycles at which the large volume of bubbles are entrained. At the end of active breaking, when the bore-like region is formed, bubble-induced dissipation becomes large and comparable to shear-induced dissipation, again. After active breaking, the total dissipation rate seems to decay like $\left(t^{*}\right)^{-n}$ where $n$ is about 2.5. In $\mathrm{S} 1, n$ is smaller during $5<t^{*}<12$, but becomes about 2.5 after $t^{*}=12$. The calculated $n$ is about $40 \%$ less than the theoretical value for isotropic turbulence, $17 / 4$. $\mathbf{R M}$ also estimated $\varepsilon \propto\left(t^{*}\right)^{-n}$ with $n=2.5$. As we expect, the viscous dissipation rate is small compared to the SGS dissipation rate.

Total breaking-induced viscous, $\hat{\epsilon}_{r}$, and SGS dissipation, $\hat{\epsilon}_{s g s}$, per unit length of crest can be calculated using

$$
\begin{aligned}
\hat{\epsilon}_{r}\left(t^{*}\right) & =\int_{t_{0}^{*}}^{t^{*}} \int_{A} \epsilon_{r} d A d t^{*}=\int_{t_{0}^{*}}^{t^{*}} \int_{A} \overline{\alpha \rho \varepsilon_{r}} d A d t^{*}, \\
\hat{\epsilon}_{s g s}\left(t^{*}\right) & =\int_{t_{0}^{*}}^{t^{*}} \int_{A} \epsilon_{s g s} d A d t^{*}=\int_{t_{0}^{*}}^{t^{*}} \int_{A} \overline{\alpha \rho \varepsilon_{s g s}} d A d t^{*},
\end{aligned}
$$

where $t_{0}^{*}=-0.25$ and $\alpha \rho$ is the density of the liquid phase at each grid point. Figure 

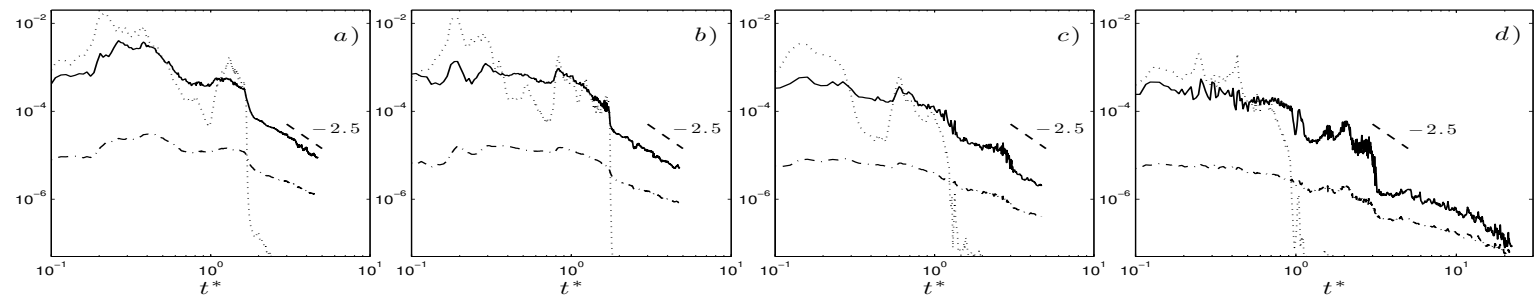

Figure 4.29: Dissipation rate per unit length of crest due to the different mechanisms for a) P1;b) P2; c) SP1 and $d$ ) S1. — shear-induced $\overline{\varepsilon_{S I}} / L_{c}^{2}, \ldots \ldots$ bubble-induced $\overline{\varepsilon_{B I}} / L_{c}^{2}$, and $-\cdot-$ viscous dissipation $\overline{\varepsilon_{r}} / L_{c}^{2}$. Dashed lines indicate $\left(t^{*}\right)^{n}$ where $n$ is the number on the lines.
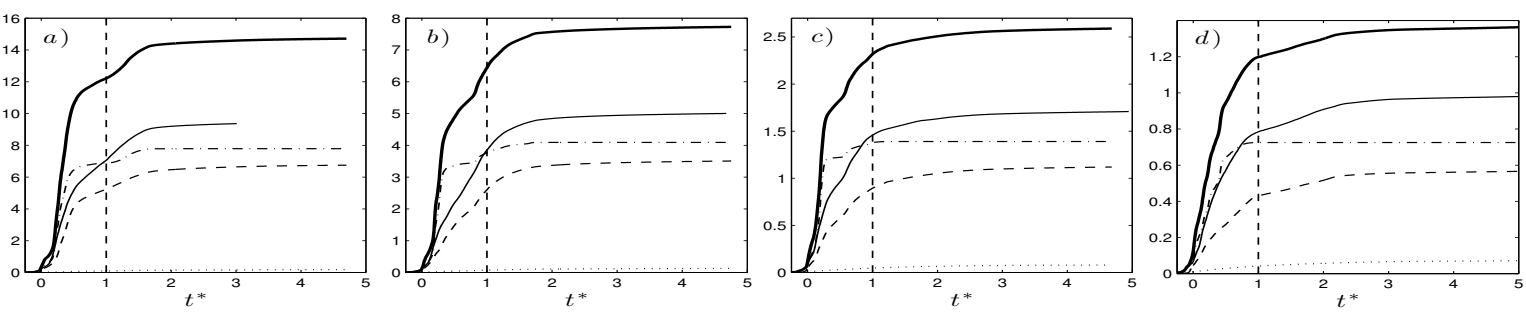

Figure 4.30: Total breaking-induced dissipation per unit length of crest $(\mathrm{J} / \mathrm{m})$ for a) P1;b) P2; c) SP1 and $d) \mathrm{S} 1 .-\hat{\epsilon}_{\text {total }}=\hat{\epsilon}_{r}+\hat{\epsilon}_{s g s}, \quad--\hat{\epsilon}_{s g s}^{S I}$, $-\cdot-\hat{\epsilon}_{s g s}^{B I}, \cdots \cdots \hat{\epsilon}_{r},-\hat{\epsilon}_{\text {total }}^{n b}$ is the total dissipation from the simulations without the inclusion of the dispersed bubbles.

4.30 shows the results for $\mathrm{P} 1, \mathrm{P} 2, \mathrm{SP} 1$ and $\mathrm{S} 1$. In all the breaker types, most of the energy is dissipated (more that $80 \%$ of the total dissipation) during the first period after breaking, with bubble-induced dissipation bigger than the shear-induced dissipation. Table 4.1 summarizes the different dissipation contributions predicted by the model and the estimation from LM. Comparing to the experimental estimations, we can conclude that the simulations without the inclusion of the dispersed bubbles underpredict total dissipation by about $35 \%$ both in plunging and spilling breaking. In addition, bubbleinduced dissipation accounts more than $50 \%$ of the total breaking induced dissipation regardless of the breakers type and intensity. We should note that the cavity break-up process is not resolved in our simulations, and may be responsible for an additional dissipation during the initial stage of active breaking. 
Table 4.1: Total dissipation in the breaking region. LM approximated the total dissipation by estimating the total energy flux difference between upstream and downstream of the breaking region.

$\begin{array}{lccccc}\text { Case no. } & \text { LM }(J / m) & \hat{\epsilon}_{\text {total }}(J / m) & \hat{\epsilon}_{\text {total }}^{n b} / \hat{\epsilon}_{\text {total }}(\%) & \hat{\epsilon}_{\text {sgs }}^{S I} / \hat{\epsilon}_{\text {total }}(\%) & \hat{\epsilon}_{\text {sgs }}^{B I} / \hat{\epsilon}_{\text {total }}(\%) \\ \text { P1 } & 17.8 & 14.7 & 63.7 & 45.9 & 52.9 \\ \text { P2 } & 8.6 & 7.7 & 64.8 & 45.4 & 53.0 \\ \text { SP1 } & 4.3 & 2.6 & 65.9 & 43.2 & 53.7 \\ \text { S1 } & & 1.4 & 64.5 & 42.3 & 51.6\end{array}$

\subsubsection{Time dependent breaking parameter, $b$}

The available estimates of breaking parameter $b$ ranges from $O\left(10^{-4}\right)$ to $O\left(10^{-2}\right)$, depending on breaking type and intensity, the unsteady or quasi-steady character, and probably the method of defining breaking parameter. In most of the previous experiments, the total breaking-induced dissipation per unit length of breaking crest, $\hat{\epsilon}_{\text {total }}$, was approximated through surface elevation measurements at fixed locations upstream and downstream of wave breaking and by implementation of a wave theory and a simple control volume analysis. The averaged dissipation rate, $\overline{\bar{\epsilon}}_{\text {total }}$, then, is defined as $\overline{\bar{\epsilon}}_{\text {total }}=\hat{\epsilon}_{\text {total }} / \tau_{b}$ where $\tau_{b}$ is a time scale related to active breaking period and is on the order of breaking wave period, $T$ - note that $(\hat{)},(\check{)}$ and $\overline{\overline{()}}$ represent time and space integration, space integration and time averaging, respectively. Finally, time averaged breaking parameter, $\overline{\bar{b}}$, is defined as

$$
\overline{\bar{b}}=\frac{g \overline{\overline{\tilde{\epsilon}}}_{t o t a l}}{\rho c^{5}} .
$$

For example, Drazen et al. (2008) estimated $\hat{\epsilon}_{\text {total }} \approx-\Delta F_{b}=\rho g C_{g_{s}} \int_{t_{1}}^{t_{2}}\left(\eta_{2}^{2}-\eta_{1}^{2}\right) d t$ where $C_{g_{s}}$ is the spectrally weighted group velocity. They chose $\tau_{b}$ equal to the acoustically active period as measured by a hydrophone.

Here, we have time dependent information of breaking-induced dissipation rate. 

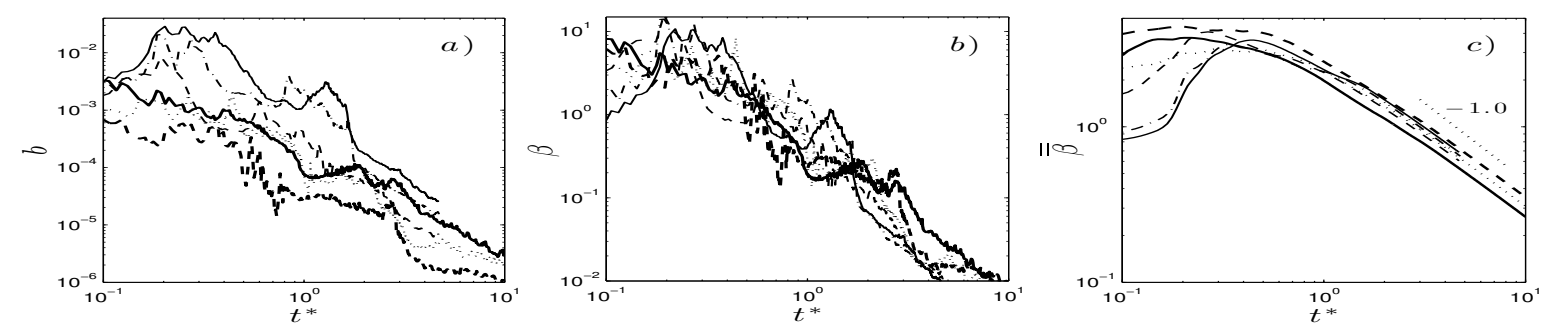

Figure 4.31: Different breaking parameters $a) b ; b) \beta$ and $c) \overline{\bar{\beta}}$ for $-\mathrm{P} 1$; - - $\mathrm{P} 2 ;---\mathrm{SP} 1 ; \ldots . \mathrm{S} 1 ;-\mathrm{P} 3$ and $---\mathrm{S} 2$.

Thus, the time dependent breaking parameter, $b$, can be readily calculated as

$$
b=\frac{g \check{\epsilon}_{t o t a l}}{\rho c^{5}} .
$$

Figure 4.31(a) shows $b$ for the different breakers. For all breakers, $b$ decreases about an order of magnitude at the end of active breaking compared to the initial stage of breaking. As expected, the decay rate of $b$ is smaller at the spilling breakers. In addition, it is clear that $b$ is strongly linked to the breaker intensity, e.g. the corresponding $b$ for P1 is about two order of magnitude greater than for S2. Intuitively, the most important parameter which can be related to breaker intensity is wave steepness. This is also confirmed by experimental measurements for both constant-amplitude and constantsteepness wave packets. Note that, P1, P2, SP1 and S1 are constant-steepness type packets with $S_{0} \approx 0.34$ while P3 and S2 are constant-amplitude type packets with $S_{0} \approx 0.25$, where $S_{0}$ is the global steepness of incipient breaking. The results reveal that the rate of increase of the breaking-induced dissipation in the packets with $S_{0}=0.34$ is considerably greater than the packets with $S_{0}=0.25$. Furthermore, the linear dependence of breaking-induced dissipation on $S-S_{0}$ is observed for the cases with the same $S_{0}$. This trend also can be recognized in DM figure 8 in which the normalized change in energy flux across the control volume increases more noticeably as $S_{0}$ increases slightly. Thus, we define

$$
b=\beta\left(S-S_{0}\right) S_{0}^{\alpha}
$$


where $\beta$ is a time dependent parameter and $\alpha$ is a constant. Figure 4.31(b) shows the resultant $\beta$ with the choice of $\alpha=4$, for all the breakers. As we can see, the choice of $\alpha=4$ successfully confines the large variation of breaking parameter for the different breakers (figure 4.31(a)) and the resultant $\beta$ has the same trend and values regardless of the breakers type and intensity.

Usually, using a time averaged value of breaking parameter, $\overline{\bar{b}}$, is more preferable in models with relatively large time scales, e.g. wind-wave models. We define

$$
\overline{\bar{b}}\left(t^{*}\right)=\frac{1}{t^{*}-t_{0}^{*}} \int_{t_{0}^{*}}^{t^{*}} b d t^{*}=\frac{1}{t^{*}-t_{0}^{*}} \int_{t_{0}^{*}}^{t^{*}} \beta\left(S-S_{0}\right) S_{0}^{4} d t^{*}=\overline{\bar{\beta}}\left(t^{*}\right)\left(S-S_{0}\right) S_{0}^{4}
$$

where $t_{0}^{*}=-0.1$. Figure $4.31(\mathrm{c})$ shows $\overline{\bar{\beta}}$ is nearly invariant for the different plunging and spilling breakers, after $t^{*}=0.3$. The appropriate $\check{\epsilon}_{\text {total }}$ in (4.16) should represent the averaged dissipation rate per unit length of breaking crest during the active bubble entrainment period which is on the order of breaking wave period. Thus, by choosing $\overline{\bar{\beta}}\left(t^{*}=0.75\right)=3$, the averaged breaking parameter becomes

$$
\overline{\bar{b}} \cong 3\left(S-S_{0}\right) S_{0}^{4}
$$

Finally, the averaged breaking-induced dissipation rate per unit length of breaking crest in the active breaking period can be approximated as

$$
\overline{\bar{\epsilon}}_{\text {total }} \cong 3 \rho g^{-1} c^{5}\left(S-S_{0}\right) S_{0}^{4}, \quad \text { for } \quad S>S_{0},
$$

and for $S<S_{0}$ the packet is non-breaking and $\overline{\bar{\epsilon}}_{\text {total }}=0$.

\subsection{Liquid-Bubble Momentum Exchange And Production By The Dis- persed Bubbles}

Momentum exchange between the dispersed bubble phase and the liquid phase through drag, lift and virtual mass forces (2.19) is examined here. Using (2.17) and 
(2.18), the total spanwise averaged, $\check{F}_{i, m}^{\text {type }}$, and r.m.s. of the turbulent forces, $\check{F}_{i, r m s}^{\text {type }}$, exerted on the water column by the bubbles are calculated as

$$
\check{F}_{i, m}^{\text {type }}=\sum_{k=1}^{N G} \int_{A}-\bar{f}_{i, k}^{\text {type }} d A, \quad \check{F}_{i, r m s}^{\text {type }}=\sum_{k=1}^{N G} \int_{A}\left(f_{i, k}^{\text {type }}\right)_{r m s} d A,
$$

where $i=1,2,3$ refer to $x, z$ and $y$ direction, respectively; and type refers to the drag, virtual mass or lift forces. Figures 4.32 and 4.33 show $\check{F}_{i, m}^{\text {type }}$ and $\check{F}_{i, r m s}^{\text {type }}$ for $\mathrm{P} 1$ and SP1. In both cases, the drag force is the main interfacial feedback in the vertical direction, and the spanwise averaged forces in the spanwise direction are an order of magnitude smaller than in the other two directions. The main interphase exchange in the streamwise direction occurs at the bubble entrainment period $\left(t^{*}=0 \sim 0.6\right.$ for $\mathrm{P} 1$ and $t^{*}=0 \sim 0.3$ for SP1), with $\check{F}_{x, m}^{D} \approx \check{F}_{x, m}^{V M}$. In the vertical direction, on the other hand, $\check{F}_{x, m}^{D} \gg \check{F}_{x, m}^{V M}$ and the spanwise averaged drag force are noticeable during the whole active breaking period.

The rate of total work done (or production rate) by the drag, virtual mass and lift forces on the organized flow, $\check{B}_{i, \text { type }}^{m}$ and the turbulent motions, $\check{B}_{i, \text { type }}^{k}$, are calculated as

$$
\check{B}_{i, \text { type }}^{m}=\sum_{k=1}^{N G} \int_{A}-\bar{f}_{i, k}^{\text {type }} \bar{u}_{i} d A, \quad \check{B}_{i, \text { type }}^{k}=\sum_{k=1}^{N G} \int_{A}-\overline{f_{i, k}^{\prime t y p e} u_{i}^{\prime}} d A .
$$

Figures 4.34 and 4.35 show the normalized $\check{B}_{i, \text { type }}^{m}$ and $\check{B}_{i, \text { type }}^{k}$ for P1 and SP1, respectively. In both cases, $\check{B}_{x} \gg \check{B}_{z} \gg \check{B}_{y}$. During the entrainment period $\left(t^{*}=0 \sim\right.$ 0.6), $\check{B}^{k}>\check{B}^{m}$ and the work done by the drag and virtual mass forces are comparable. The drag force mostly damps the turbulence and organized flow, while the virtual mass force enhances them. After $t^{*} \sim 0.6$, on the other hand, the total work done on turbulence decreases considerably and the work done by the drag force on the organized flow becomes the most noticeable feedback.

The total work done by the interfacial forces on the organized flow and the 

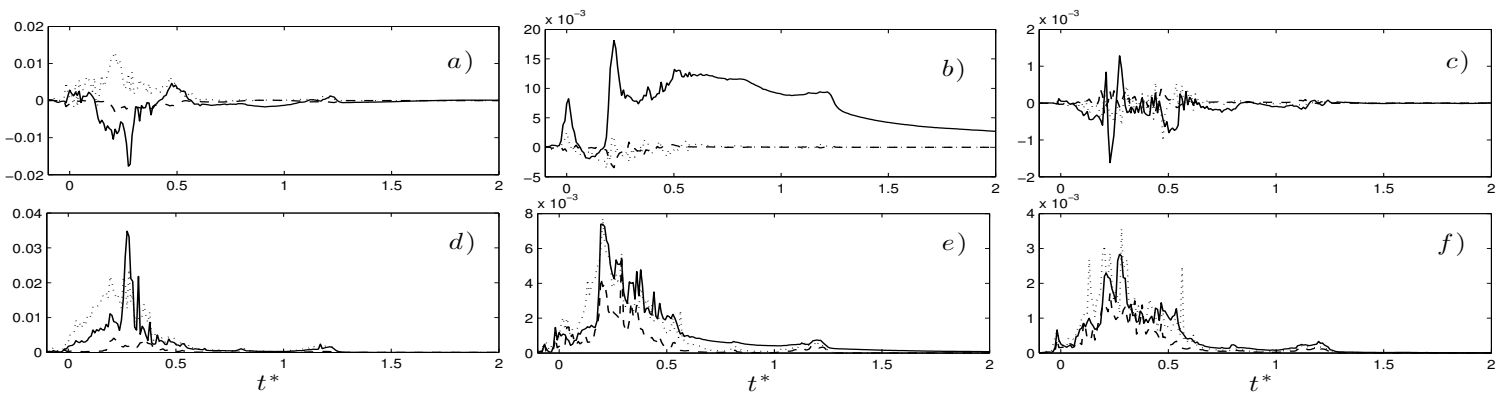

Figure 4.32: Total spanwise averaged and r.m.s. of the interfacial forces exerted on the water column by the bubbles, $(a, b, c) \check{F}_{i, m}^{\text {type }}$ and $(d, e, f) \check{F}_{i, r m s}^{\text {type }}$ for P1. $(a, d)$ streamwise direction; $(b, e)$ vertical direction; $(c, f)$ spanwise direction. — drag force, $\cdots \cdots$ virtual mass force, --- lift force. The reference value is $\rho L_{c}^{2} C_{c} T_{c}^{-1}$.
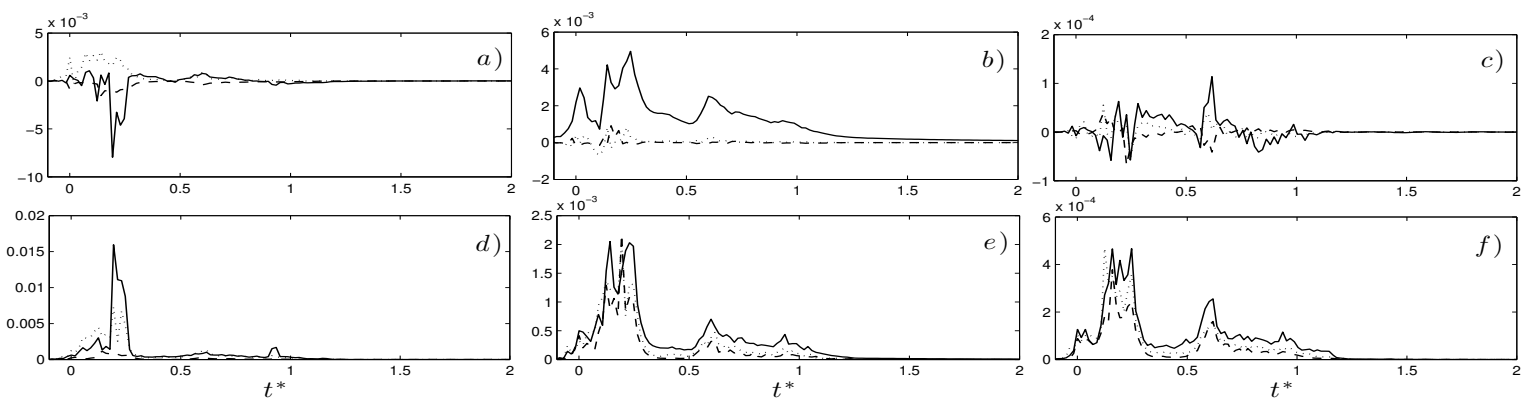

Figure 4.33: $(a, b, c) \check{F}_{i, m}^{\text {type }}$ and $(d, e, f) \check{F}_{i, r m s}^{\text {type }}$ for SP1. The definitions are the same as figure 4.32 .
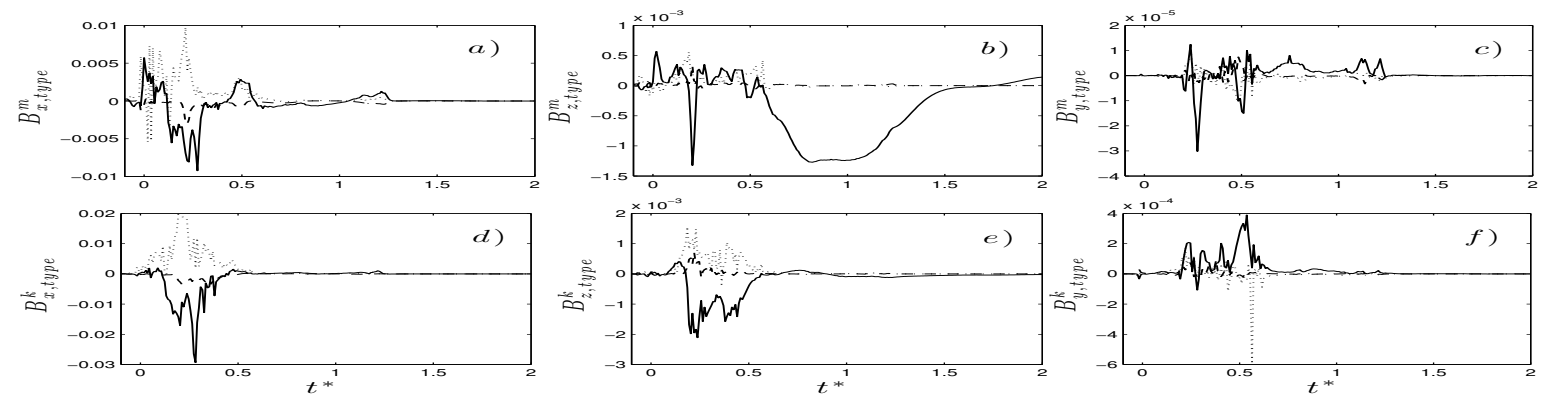

Figure 4.34: The rate of work done on the organized and turbulent flow by the dispersed bubbles, $(a, b, c) \check{B}_{i, t y p e}^{m}$ and $(d, e, f) \check{B}_{i, \text { type }}^{k}$ for $\mathrm{P} 1 . \quad(a, d)$ streamwise direction; $(b, e)$ vertical direction; $(c, f)$ spanwise direction. drag force, $\cdots \cdots$ virtual mass force, --- lift force. The reference value is $\rho L_{c}^{2} C_{c}^{2} T_{c}^{-1}$. 

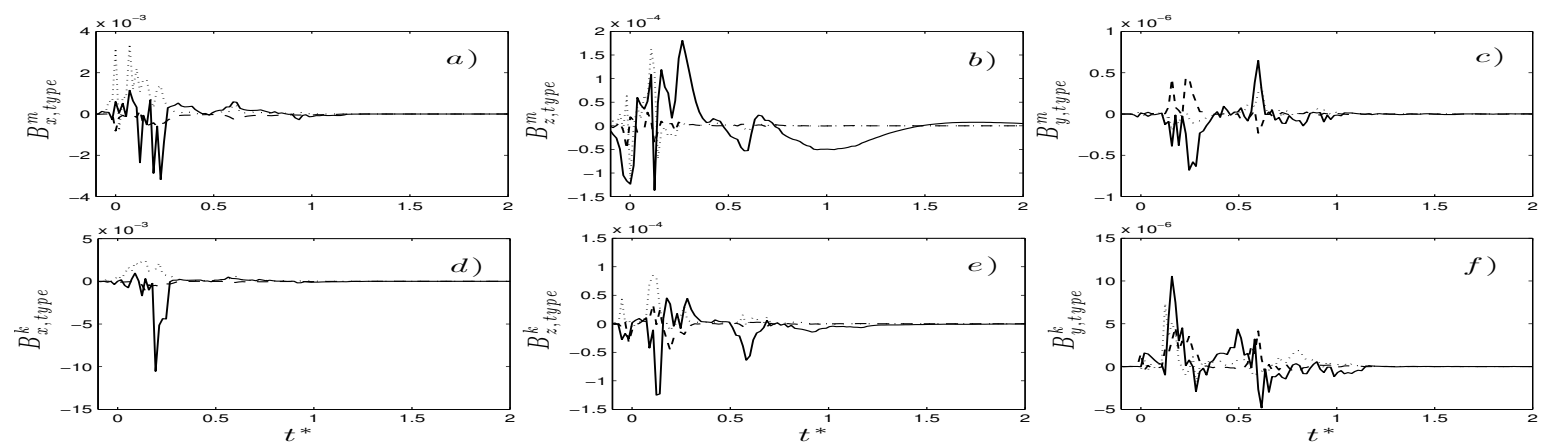

Figure 4.35: $(a, b, c) \check{B}_{i, t y p e}^{m}$ and $(d, e, f) \check{B}_{i, \text { type }}^{k}$ for SP1. The definitions are the same as figure 4.34 .
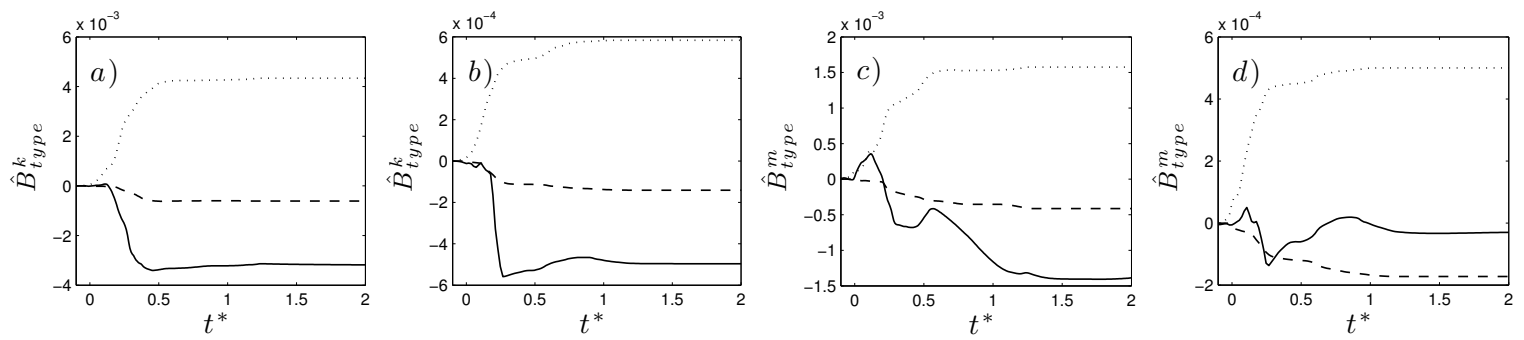

Figure 4.36: Total work done by _- drag, $\ldots \ldots$ virtual mass, and --- lift force, on $(a, b)$ turbulent motions and $(c, d)$ organized flow; for $(a, c) \mathrm{P} 1$ and $(b, d) \mathrm{SP} 1$. The reference value is $\rho L_{c}^{2} C_{c}^{2}$.
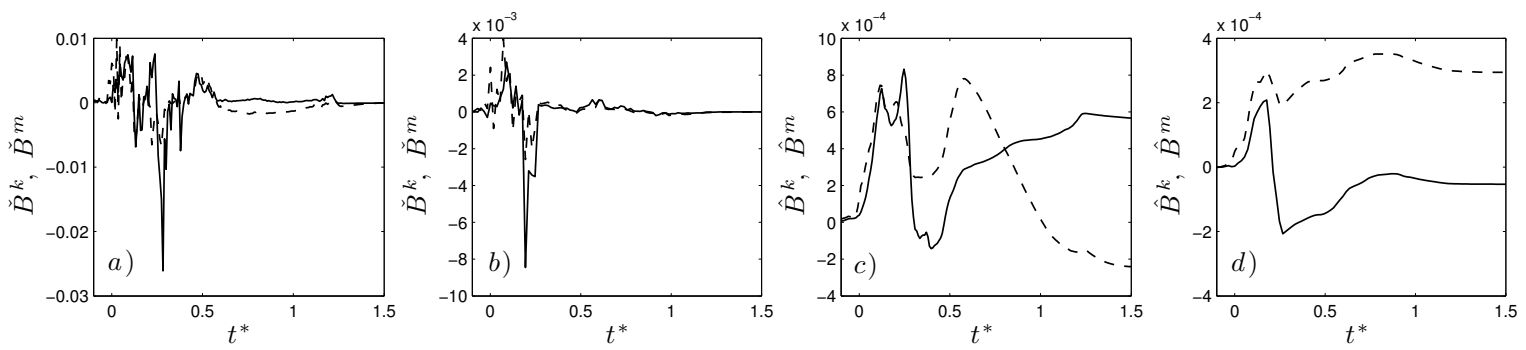

Figure 4.37: $(a, b)$ Total rate of work done by the dispersed bubbles on — TKE and - - - the organized flow for $a) \mathrm{P} 1 ; b) \mathrm{SP} 1$. The reference value is $\rho L_{c}^{2} C_{c}^{2} T_{c}^{-} 1 .(c, d)$ Total work done by the dispersed bubbles on TKE and - - the organized flow for $c$ ) P1; $d$ )SP1. The reference value is $\rho L_{c}^{2} C_{c}^{2}$. 
turbulent motions from $t_{0}^{*}$ to $t^{*}$, can be calculated as

$$
\hat{B}_{\text {type }}^{k}\left(t^{*}\right)=\sum_{i=1}^{3} \int_{t_{0}^{*}}^{t^{*}} \check{B}_{i, \text { type }}^{k} d t^{*}, \quad \hat{B}_{\text {type }}^{m}\left(t^{*}\right)=\sum_{i=1}^{3} \int_{t_{0}^{*}}^{t^{*}} \check{B}_{i, \text { type }}^{m} d t^{*}
$$

Figure 4.36 shows the total work done by the drag, virtual mass and lift forces on the organized and turbulent motions for P1 and SP1. The total work done by the drag and lift forces damp the organized flow and turbulence while the work done by the virtual mass force enhances them in both cases. The summation of the total work done by all three mechanisms, hereafter refers to the production by the dispersed bubbles, leads to a different behavior in the different breakers. Figure 4.37 shows the organized flow and TKE production by the dispersed bubbles for P1 and SP1. After the period of active breaking, say $t^{*}=1.5$, the total productions of the organized flow and TKE by the

dispersed bubbles in the breaking region, $\hat{B}^{k}\left(t^{*}=1.5\right), \hat{B}^{m}\left(t^{*}=1.5\right)$ would indicate the integral effects during active breaking. Thus, in the plunging breaker, production by the dispersed bubbles enhances the turbulence and damps the organized flow. In the spilling case, on the other hand, it damps the turbulence and enhances the organized flow.

\subsection{TKE Transport In The Breaking Region}

Figure 4.38 demonstrates that the other mechanisms, such as buoyancy production, production by mean shear and dissipation, should be considered beside the production by the dispersed bubbles, to examine turbulence modulation by dispersed bubbles under breaking waves.

In LES, the transport equation for resolved TKE can be obtained based on the resolved velocity field. SGS dissipation contains both shear- and bubble-induced dissipation and typically is much bigger than the viscous dissipation (figure 4.30). In the case of a two phase flow with a dilute regime $(\alpha \approx 1)$, common practice is to use the conventional single phase TKE transport equation with an additional term due to a 
correlation between fluctuating concentration and vertical turbulent velocity component, $-\rho g \overline{\alpha^{\prime} w^{\prime}}$. As we showed, high turbulent and dissipative regions are collocated with high void fraction regions. Due to a large void fraction $(>10 \%)$ during active breaking period, the dilute assumption is not valid anymore, and concentration fluctuations can not be ignored. The exact resolved TKE transport equation is derived in Appendix B.2. The final equation is given by

$$
\begin{aligned}
\frac{\partial}{\partial t}(\rho \bar{\alpha} \bar{k})+\frac{\partial}{\partial x_{j}}\left(\rho \bar{\alpha} \bar{u}_{j} \bar{k}\right) & =T^{k}+P^{k}+B^{k}+E^{k}-\left(\epsilon_{r}^{k}+\epsilon_{s g s}^{k}\right)+D^{k} \\
& +t_{e x}^{k}+T_{e x}^{k}+P_{e x}^{k}-\left(\epsilon_{r, e x}^{k}+\epsilon_{s g s, e x}^{k}\right)+D_{e x}^{k}
\end{aligned}
$$

where $k=\frac{1}{2} u_{i}^{\prime} u_{i}^{\prime}$ is the resolved TKE and

$$
\begin{aligned}
& P^{k}=-\rho \bar{\alpha} \overline{u_{i}^{\prime} u_{j}^{\prime}} \frac{\partial \bar{u}_{i}}{\partial x_{j}}, \quad \text { (production rate by mean shear) } \\
& \epsilon_{r}^{k}=2 \rho \nu \bar{\alpha} \overline{\mathcal{S}^{\prime}{ }_{i j} \mathcal{S}^{\prime}{ }_{i j}}, \quad \text { (viscous dissipation rate) } \\
& \epsilon_{s g s}^{k}=-\rho \bar{\alpha} \overline{\tau_{i j}^{\prime d} \mathcal{S}^{\prime}{ }_{i j}}, \quad \text { (SGS dissipation rate) } \\
& P_{e x 1}^{k}=-\rho \bar{u}_{j} \overline{\alpha^{\prime} u_{i}^{\prime}} \frac{\partial \bar{u}_{i}}{\partial x_{j}}, \quad P_{e x 2}^{k}=-\rho \overline{\alpha^{\prime} u_{i}^{\prime} u_{j}^{\prime}} \frac{\partial \bar{u}_{i}}{\partial x_{j}}, \quad \text { (extra production terms) } \\
& \epsilon_{r, e x 1}^{k}=2 \rho \nu \overline{\alpha^{\prime} \mathcal{S}^{\prime}{ }_{i j} \mathcal{S}^{\prime}{ }_{i j}}, \quad \epsilon_{r, e x 2}^{k}=2 \rho \nu \overline{\alpha^{\prime} \mathcal{S}^{\prime}{ }_{i j}} \overline{\mathcal{S}}_{i j}, \quad \text { (extra viscous dissipation terms) } \\
& \epsilon_{s g s, e x 1}^{k}=-\rho \overline{\alpha^{\prime} \tau_{i j}^{\prime d} \mathcal{S}_{i j}^{\prime}}, \quad \epsilon_{s g s, e x 2}^{k}=-\rho \overline{\alpha^{\prime} \mathcal{S}^{\prime}{ }_{i j}} \bar{\tau}_{i j}^{d}, \quad \text { (extra SGS dissipation terms). }
\end{aligned}
$$

The other terms are given by (B.8) and (B.9) in Appendix B.2. In the case of a single phase flow $\bar{\alpha}=1$ and $\alpha^{\prime}=\frac{\partial \bar{u}_{j}}{\partial x_{j}}=\frac{\partial u_{j}^{\prime}}{\partial x_{j}}=0$, by which the extra terms becomes zero and (4.28) reduces to the single phase classical transport equation for turbulent kinetic energy. For example, the single phase flow SGS dissipation rate and mean shear production rate are given by

$$
\epsilon_{s g s}^{S P}=-\rho \overline{\tau_{i j}^{\prime d} \mathcal{S}^{\prime}{ }_{i j}}, \quad P^{S P}=\overline{\rho u_{i}^{\prime} u_{j}^{\prime} \frac{\partial \bar{u}_{i}}{\partial x_{j}}}
$$




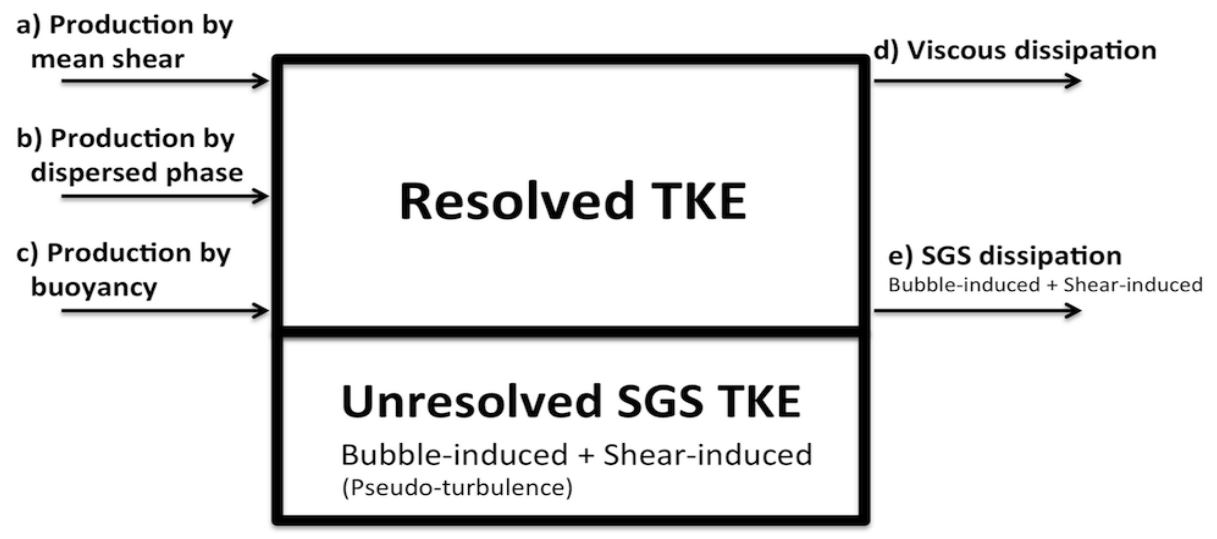

Figure 4.38: Schematic of the different production and dissipation mechanisms of two phase bubbly flow TKE.
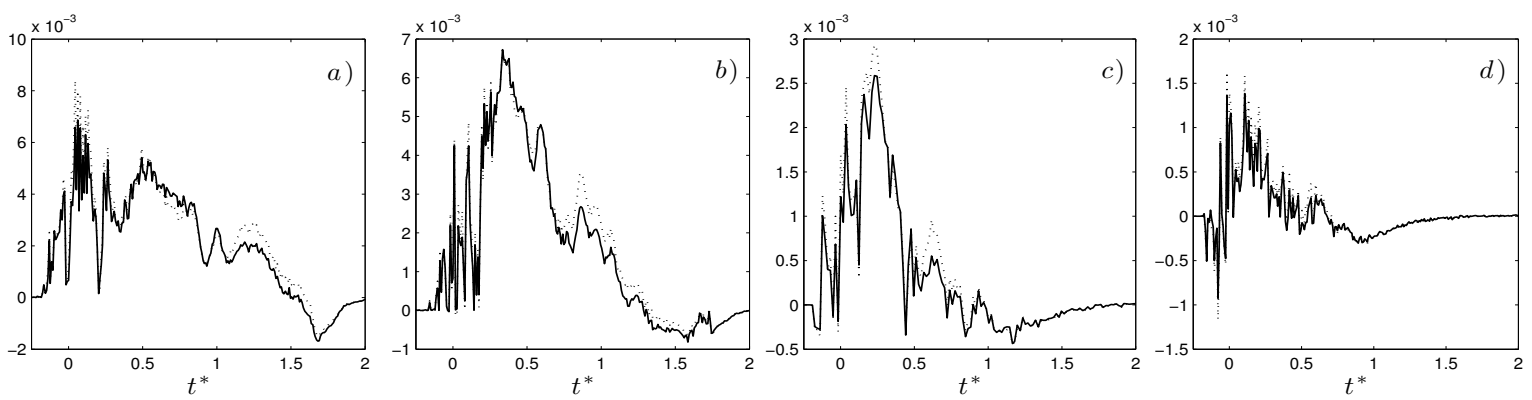

Figure 4.39: Total production rate by mean shear in the breaking region based on the two phase $-\check{P}^{k}+\check{P}_{e x 1}^{k}+\check{P}_{e x 2}^{k}$ and the single phase transport equation $\cdots . . \check{P}^{S P}$, for $\left.a\right) \mathrm{P} 1$; b) P2; c) SP1 and $d$ ) S1. The reference value is $\rho L_{c}^{2} C_{c}^{2} T_{c}^{-1}$.
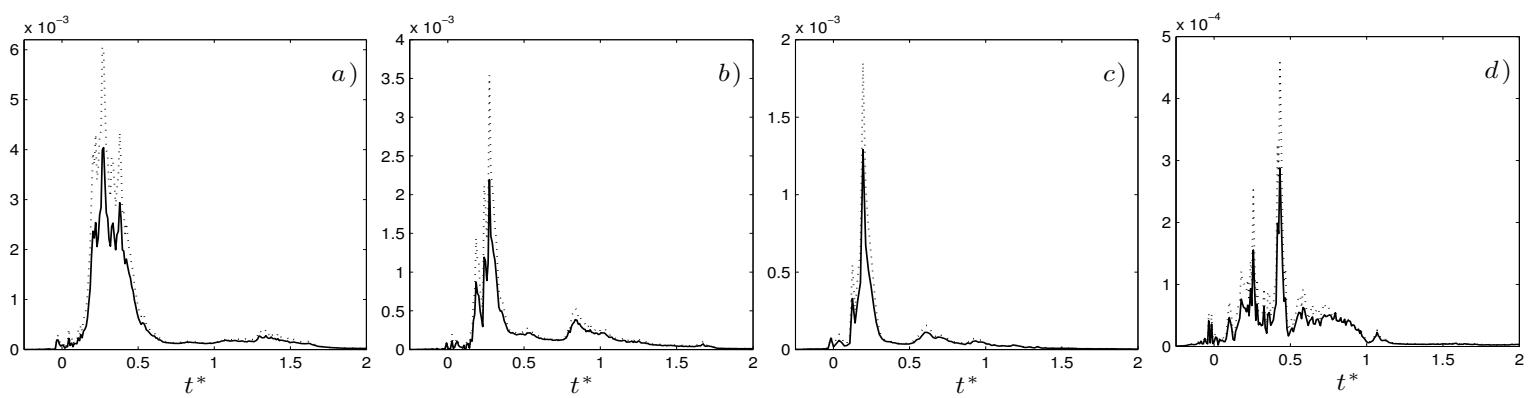

Figure 4.40: Total SGS dissipation rate in the breaking region based on the two phase $-\check{\epsilon}_{s g s}^{k}+\check{\epsilon}_{s g s, e x 1}^{k}+\check{\epsilon}_{s g s, e x 2}^{k}$ and the single phase transport equation $\ldots . \quad \check{\epsilon}_{s g s}^{S P}$ for $\left.\left.\left.a\right) \mathrm{P} 1 ; b\right) \mathrm{P} 2 ; c\right) \mathrm{SP} 1$ and $\left.d\right) \mathrm{S} 1$. The reference value is $\rho L_{c}^{2} C_{c}^{2} T_{c}^{-1}$. 

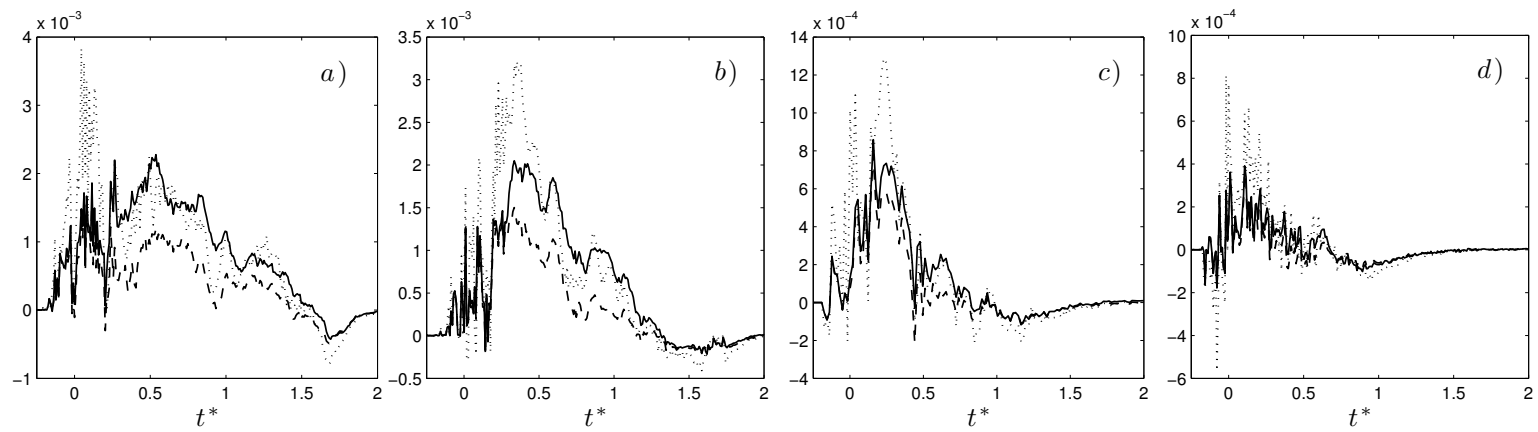

Figure 4.41: Different terms in the total production rate by mean shear $-\check{P}^{k}$; $--\check{P}_{e x 1}^{k}$ and $\ldots . . \check{P}_{e x 2}^{k}$ for $\left.\left.\left.a\right) \mathrm{P} 1 ; b\right) \mathrm{P} 2 ; c\right) \mathrm{SP} 1$ and $\left.d\right) \mathrm{S} 1$. The reference value is $\rho L_{c}^{2} C_{c}^{2} T_{c}^{-1}$.
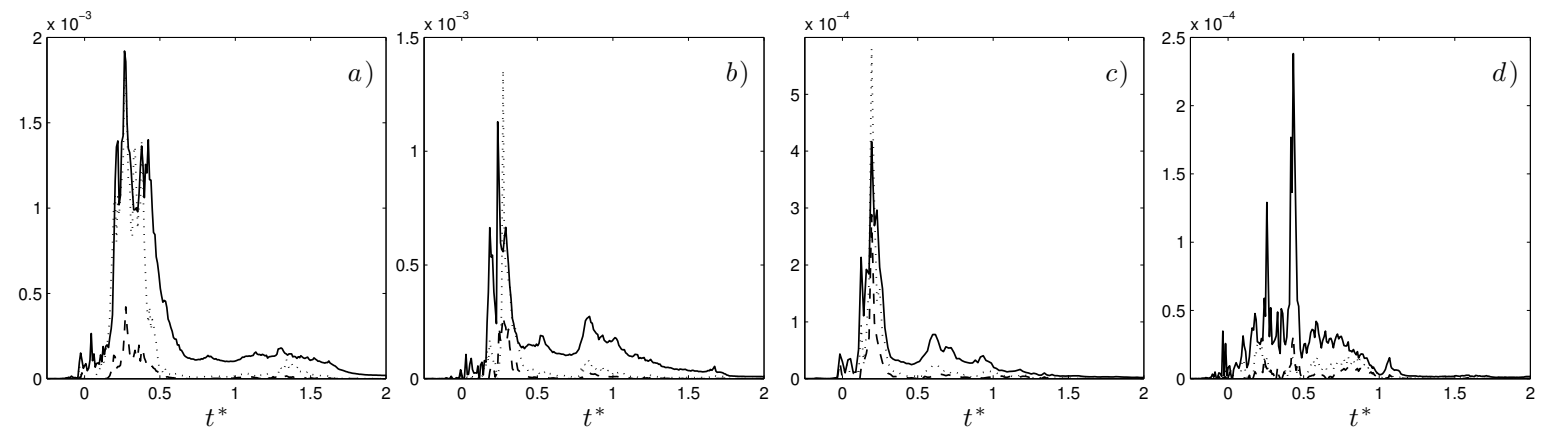

Figure 4.42: Different terms in the total SGS dissipation rate $-\check{\epsilon}_{s g s}^{k} ; \quad---\check{\epsilon}_{s g s, e x 1}^{k}$ and $\cdots . . \check{\epsilon}_{s g s, e x 2}^{k}$ for $\left.\left.a\right) \mathrm{P} 1 ; b\right) \mathrm{P} 2$; c) SP1 and $\left.d\right) \mathrm{S} 1$. The reference value is $\rho L_{c}^{2} C_{c}^{2} T_{c}^{-1}$.
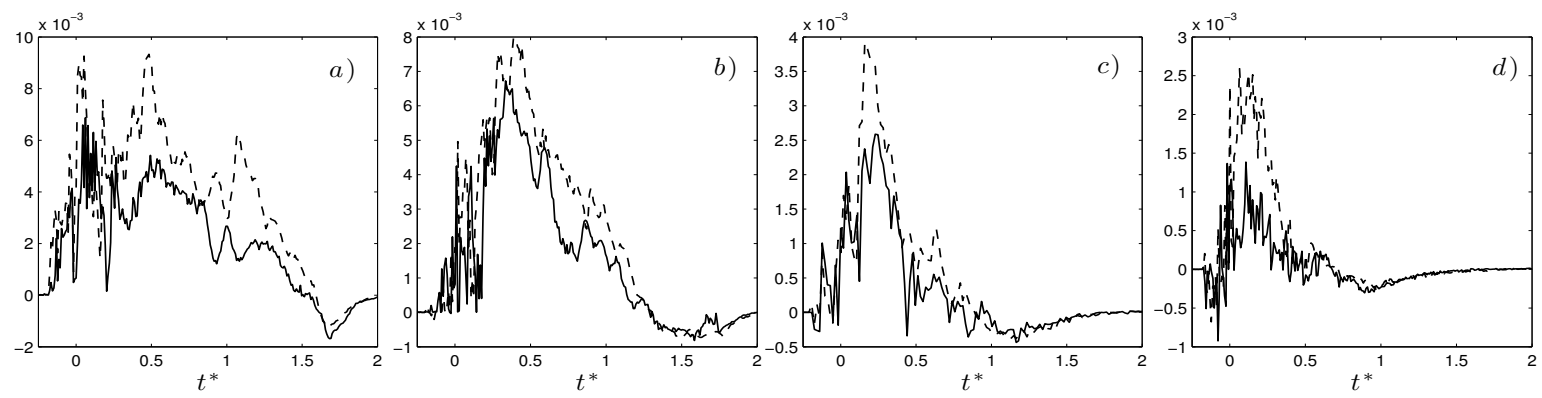

Figure 4.43: The two phase total production rate by mean shear $\check{P}_{\text {total }}^{k}=\check{P}^{k}+\check{P}_{e x 1}^{k}+$ $\check{P}_{e x 2}^{k}$ from $\_$simulation with dispersed bubbles and --- simulation without inclusion of the dispersed bubbles for a) P1;b) P2; c) SP1 and d) $\mathrm{S} 1$. The reference value is $\rho L_{c}^{2} C_{c}^{2} T_{c}^{-1}$. 

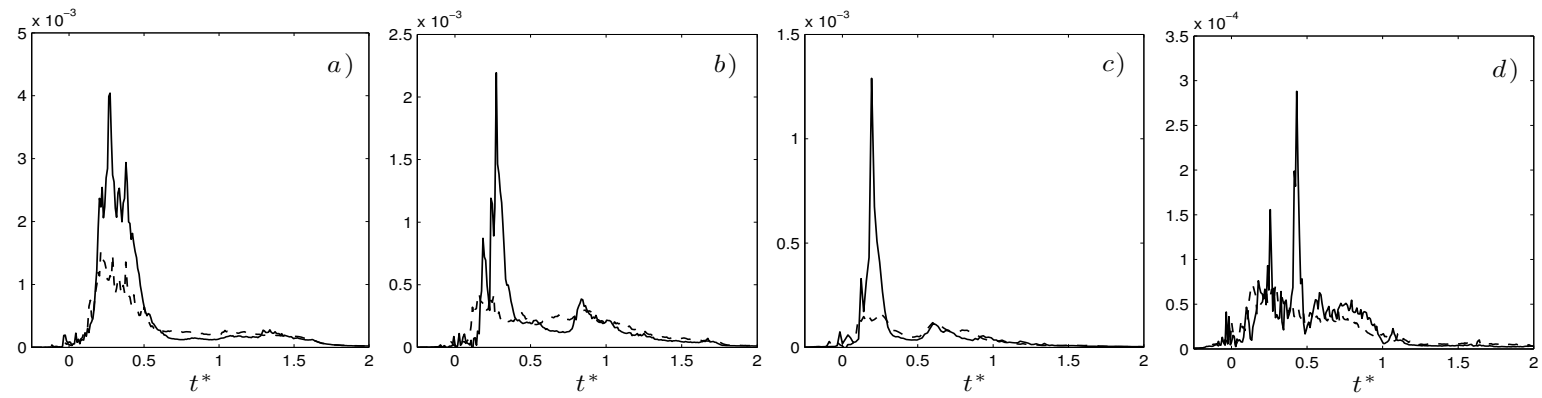

Figure 4.44: The two phase total SGS dissipation rate by mean shear $\check{\epsilon}_{\text {total }}^{k}=$ $\check{\epsilon}^{k}+\check{\epsilon}_{e x 1}^{k}+\check{\epsilon}_{e x 2}^{k}$ from simulation with dispersed bubbles and - - - simulation without inclusion of the dispersed bubbles for a) P1; b) $\mathrm{P} 2$; c) SP1 and $d) \mathrm{S} 1$. The reference value is $\rho L_{c}^{2} C_{c}^{2} T_{c}^{-1}$.
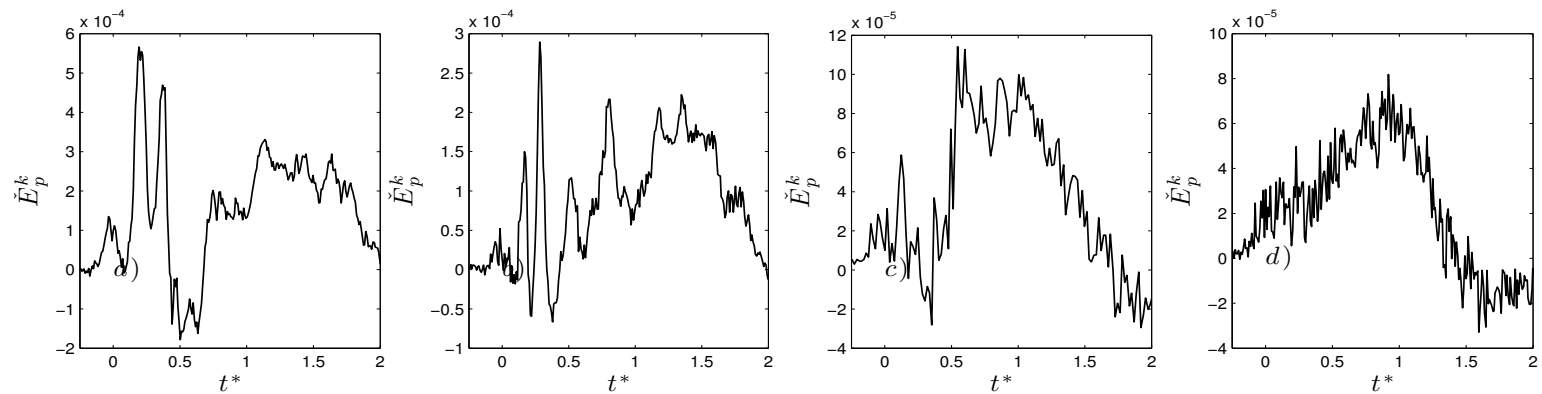

Figure 4.45: The total production by buoyancy $\check{E}_{p}^{k}$, for $a$ ) P1; b) P2; c) SP1 and $d$ ) $\mathrm{S} 1$. The reference value is $\rho L_{c}^{2} C_{c}^{2} T_{c}^{-1}$.
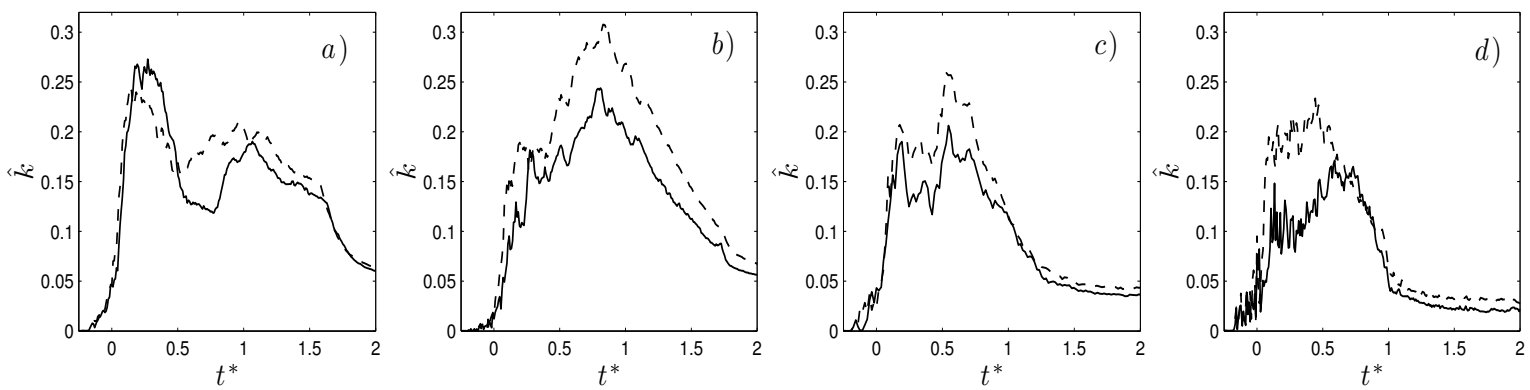

Figure 4.46: Normalized total resolved TKE, $\check{k}$, in the breaking region, — simulation with dispersed bubbles and - - - simulation without inclusion of the dispersed bubbles for $a$ ) P1; b) P2; c) SP1 and $d$ )S1. The reference value is $L_{c}^{2} C_{c}^{2}(S-S 0) S_{0}^{4}$. 


\subsubsection{The two phase vs single phase TKE transport equation}

Figures 4.39 and 4.40 show the corresponding values for the total production rate by mean shear and SGS dissipation in the breaking region using the exact two phase transport equation and the single phase transport equation. As we can see, the total SGS dissipation rate is overpredicted by about 30 to $50 \%$ by (4.30) when the void fractions are high. The total production rate by mean shear is also over predicted by the single phase formulation but the over prediction is smaller than 10\%. Figures 4.41 and 4.42 show different terms in the total production rate by mean shear and dissipation rate given by (4.29) and (eq4.30). The extra terms with triple fluctuating correlation are usually larger than the other extra term both in production and SGS dissipation rate. In addition, during the initial stage of active breaking, they are the dominant terms. The extra terms in the total SGS dissipation rate of the two phase formulation are noticeable only at the entrainment stages. In production terms, instead, $\check{P}_{e x 1}^{k}$ and $\check{P}_{e x 2}^{k}$ are comparable to $\check{P}^{k}$ in $0<t^{*}<1$.

\subsubsection{Turbulence modulation by the dispersed bubbles}

Figures 4.43 and 4.44 show the total production rate by mean shear and SGS dissipation rate for the simulations with and without the inclusion of the dispersed bubbles. The presence of dispersed bubbles reduces turbulence production rate by mean shear while enhancing the turbulence dissipation rate. The total production rate

by buoyancy $\check{E}_{p}^{k}$ is an order of magnitude smaller than the production rate by mean shear and the dispersed bubbles (figure 4.45).

Figure 4.46 shows the dispersed bubbles damped total TKE about 20 to $30 \%$ in the plunging breakers. Exception is in $0.1<t^{*}<0.5$, where TKE is damped slightly or even enhanced in the large plunging case, $\mathrm{P} 1$. This can be explained through turbulence production by dispersed bubbles in which enhances TKE to some extend. Figure 4.37 shows that bubbles enhance large scale turbulent motions through $B^{k}=-\overline{f_{i}^{\prime} u_{i}^{\prime}}$ at the entrainment stage, which compensates some portion of both the reduction of mean shear production and enhancement of dissipation during these times by the dispersed 

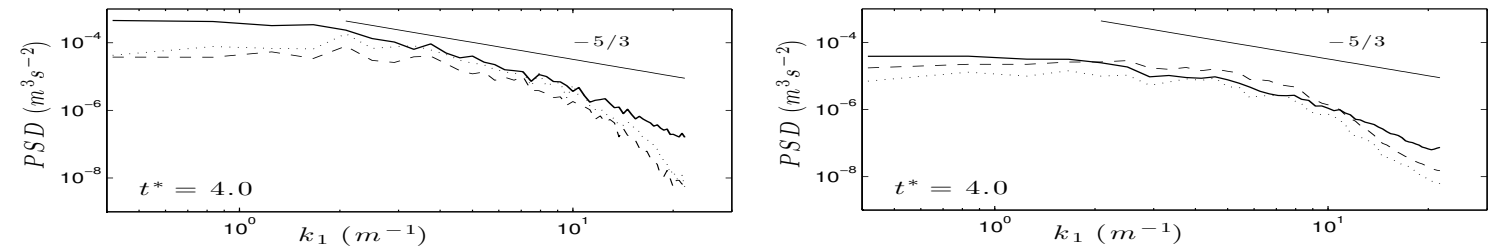

Figure 4.47: Spanwise averaged energy spectra at different elevations for P3 at $t^{*}=4$. (left) $z^{*}=-0.02$ and (right) $z^{*}=-0.1 .-E_{11} ;---E_{31}$ and $\cdots \cdots E_{21}$.

bubbles. In P1 the turbulence production by dispersed bubbles is very large, leading to the net enhancement of total TKE during $0.1<t^{*}<0.5$. In the smaller spilling breaker, S1, this production by bubbles are weak and thus, the decrease of TKE is more noticeable and about 50\%. Finally, figure 4.46 reveals that total TKE can also be scaled by $(S-S 0) S_{0}^{4}$ similar to the averaged dissipation rate (4.24).

\subsection{Velocity Spectra}

The one sided wavenumber spectra is calculated as

$$
E_{i j}\left(k_{j}, t\right)=2 N d x_{j}\left|\hat{u}_{i}\left(k_{j}, t\right)\right|^{2}
$$

where $\mathrm{N}$ is the number of grid points in the signal, $d x_{j}$ is the grid spacing in $j$ direction and $\hat{u}_{i}\left(k_{j}, t\right)$ is the FFT of $u_{i}\left(x_{j}, t\right)$. To calculate $E_{i 1}$, the velocity signals are selected between $0.25<x^{*}<1.5$ and windowed using a Tukey window with a taper ratio of 0.2. Note that after $t^{*}=4$ the packet has passed the breaking region. As we can see in figure 4.47 , close to the free surface $\left(z^{*}=-0.02\right), E_{31}<E_{11} \approx E_{21}$ over the whole range of scales, consistent with Shen \& Yue (2001) who also found anisotropy in the surface layer at all length scales. Far from the free surface $\left(z^{*}=-0.1\right)$, instead, the turbulent fluctuations in the vertical direction is stronger than or comparable with the other two components.

Figure 4.48 demonstrates the energy spectra at $t^{*}=1$ in the spanwise direction in the turbulent and non-turbulent regions for P1. $z^{*}=-0.05, x^{*}=0.45$ located 

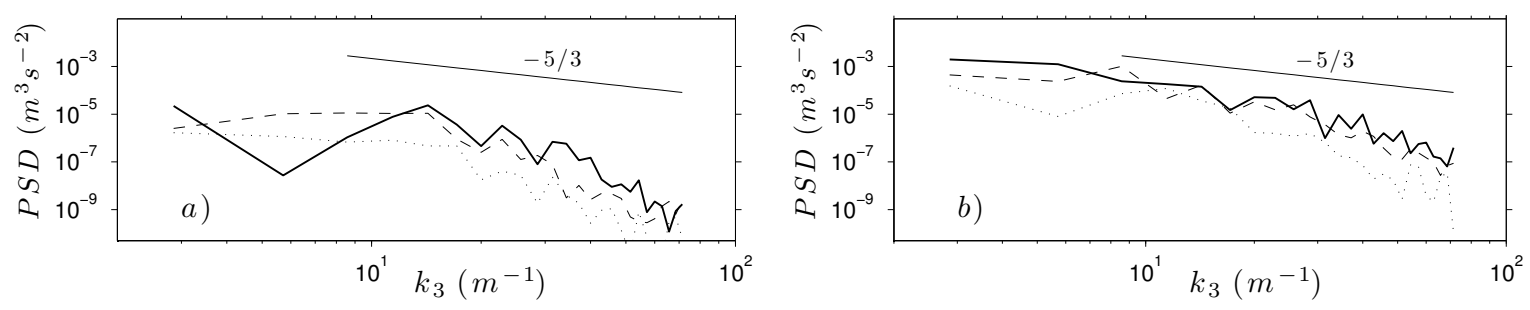

Figure 4.48: Energy spectra at $t^{*}=1, z^{*}=-0.05$ and different $x$ locations for P1. a) out side the turbulent region, $x^{*}=0.2$ and $b$ ) inside the turbulent region, $x^{*}=0.45$. $-E_{12} ;---E_{32}$ and $\cdots \cdots E_{22}$.

inside the high TKE region and the other point, $z^{*}=-0.05, x^{*}=0.2$, located at the region with negligible turbulent fluctuations. As we can see, the spectrum changes to $3 \mathrm{D}$ turbulence shape with the $-5 / 3$ slope in the high TKE region. 


\section{Chapter 5 CONCLUSION}

Continuous poly-disperse two-fluid model was used to study the turbulent bubbly flow under a laboratory scale isolated deep water breaking waves imposed by the focused wave method. Bubbles were entrained at the free surface using the bubble entrainment model. The free surface was captured by the second order VOF interface tracking scheme. Turbulence was simulated using a LES approach with dynamic Smagorinsky subgrid formulation. The SGS bubble-induced turbulence and dissipation as well as momentum transfer between two phases were considered. The main conclusions can be summarized in the following categories:

(a) Bubble entrainment and transport: It was shown that the entrainment model can predict the correct volume of entrained bubbles during the jet/splash impacts and in the bore-like region for the plunging and plunging/spilling breakers. The initial cavity entrapment is not a turbulence related entrainment mechanism and can be captured by the VOF model. Although we do not have any detailed measurement for the purely spilling case to compare with, we can expect that the entrainment model also predicts the correct volume of entrained air for spilling breakers during the entire entrainment period, in which bubble entrainment in the bore-like region is the dominant entrainment mechanism. Comparing snapshots of the void fraction distributions as well as integral properties of the bubble plumes to the corresponding experiments, we can conclude that the model captures the spatial and temporal evolution of entrained bubbles fairly accurately. 
(b) Nonlinear interaction of wave packet components: For the first time, phase locking between spectral components was observed in the breaking region, and explained by calculating the wavelet bispectrum. Phase coupling starts near the break point and lasts for more than two periods.

(c) Velocity and vorticity field: Spanwise averaged velocities were decomposed into mean current, surface waves and forced long waves. The current induced by breaking is noticeable about one wavelength downstream of the breaking point. The positive current penetrates more than a wave height with the maximum near the still water depth. The nearly constant return current compensates the positive current below the still water depth. The large coherent breaking-induced vortex, previously observed by RM and DM, was captured by the model. Based on the penetration of the TKE and enstrophy, the mixed layer depth was estimated to be $0.5 H$ for the spilling and $0.75 H$ for the plunging breakers during active breaking, where $H$ is the wave height before breaking. It becomes about twice deeper at $t^{*}=4.0$, with $1 H$ for the spilling and $1.5 \mathrm{H}$ for the plunging breakers. In P1, the mixed layer depth further increases to about $2.5 H$ at $t^{*}=11.0$. At later times, the averaged layer depth remains nearly constant. During active breaking, the turbulent velocity and vorticity field in the near-surface layer were shown to be very anisotropic both in the spilling and plunging breakers. In the plunging breakers, the anisotropy reduces as time proceeds, and the vorticity component in the streamwise direction becomes comparable and even larger than the other two components. In the spilling breakers, on the other hands, the anisotropy both in the turbulent velocity and vorticity field remains noticeable after active breaking.

The decay rate of both total TKE, $\check{\bar{k}}$, and total enstrophy, $\check{\bar{\Omega}}$, increase with increasing breaking intensity. After active breaking, $\check{k}$ decays in the form of $\left(t^{*}\right)^{-n}$ in which $n$ varies from 0.5 to 1.0 for spilling to plunging breaking. $\check{\Omega}$ has a similar decay form with larger $n$ varies from 1.0 to 1.5 for spilling to plunging breaking.

(d) Dissipation: In all the breaker types, most of the energy (more than $80 \%$ of 
the total dissipation) is dissipated during the first period after breaking. The bubbleinduced dissipation accounts about $50 \%$ of the total dissipation. The bubble-induced dissipation rate is very noticeable during the jet/splash cycles at which the large volume of bubbles are entrained. At the end of active breaking, when the bore-like region is formed, the bubble-induced dissipation rate becomes large and comparable to the shear-induced dissipation rate, again. After active breaking, the total dissipation rate was shown to decay like $\left(t^{*}\right)^{-n}$ where $n$ is about 2.5 .

In addition, the averaged dissipation rate per unit length of breaking crest is usually written as $b \rho g^{-1} c^{5}$, where $c$ is the phase speed of the breaking wave. The breaking parameter, $b$, has been poorly constrained by experiments and field measurements. Time dependent evolution of $b$ was examined for both constant-steepness and constant-amplitude wave packets. The scaling law for the averaged breaking parameter was obtained as $\overline{\bar{b}} \cong 3\left(S-S_{0}\right) S_{0}^{4}$ where $S=\Sigma a_{i} k_{i}$ is the global steepness of the packet and $S_{0}$ is the global steepness of incipient breaking.

(e) Liquid-bubble momentum exchange: In both spilling and plunging breakers, the drag force is the main interfacial feedback in the vertical direction. In the streamwise direction, the momentum exchange is noticeable during the initial stage of the active breaking with the spanwise averaged total drag and virtual mass forces are at the same order but in the opposite direction. The total work done by the drag and lift forces damp the organized flow and turbulence while the work done by the virtual mass force enhances them in both the spilling and plunging breakers.

(f) The two phase vs single phase TKE transport equation: Due to a large void fraction $(>10 \%)$ during active breaking, the dilute assumption is not valid anymore, and concentration fluctuations can not be ignored. The exact two-phase transport equation for TKE was derived. It was found that in the high void fraction regions, SGS dissipation rate is over predicted about 30 to $50 \%$ by the single phase TKE transport equation. The total production rate by mean shear is also over predicted by the single phase formulation but the over prediction is smaller than $10 \%$. The extra terms with triple fluctuating correlation are usually larger than the other extra term 
both in production and SGS dissipation rate in the two-phase TKE transport equation. (g) Turbulence modulation by dispersed bubbles: All of the 3D simulations are repeated without the inclusion of dispersed bubble phase, and it is shown that the integrated TKE in the breaking region is damped by the dispersed bubbles about $20 \%$ for the large plunging breaker to $50 \%$ for the spilling breaker. In the plunging breakers, TKE is damped slightly or even enhanced during the initial stage of active breaking. This was explained through the noticeable turbulence production by the dispersed bubbles in the larger plunging breakers during the initial stage of active breaking. 


\section{BIBLIOGRAPHY}

Banner, M. L. \& Peregrine, D. H. 1993 Wave breaking in deep water. Ann. Rev. Fluid Mech. 25, 373-397.

Blenkinsopp, C. E. \& Chaplin, J. R. 2007 Void fraction measurements in breaking waves. Proc. Roy. Soc. A 463, 3151-3170.

Callaghan, A H, Deane, G B \& D. Stokes, M 2013 Two regimes of laboratory whitecap foam decay: bubble-plume controlled and surfactant stabilized. J. Phys. Oceanogr. 43 (1114-1126).

Carrica, P. M., Drew, D., Bonetto, F. \& Lahey, R. T. 1999 A polydisperse model for bubbly two-phase flow around a surface ship. Int. J Multiphase Flow 25, $257-305$.

Chen, G., Kharif, Ch., Zaleski, S. \& Li, J. 1999 Two-dimensional navier-stokes simulation of breaking waves. Phys. Fluids 11, 121-133.

Christensen, E. D. 2006 Large eddy simulation of spilling and plunging breakers. Coastal Eng. 53, 463-485.

Christensen, E. D. \& DeigaArd, R. 2001 Large eddy simulation of breaking waves. Coastal Eng. 42, 53-86.

Clift, R., Grace, J. R. \& Weber, M. E. 1978 Bubbles, Drops and Particles, Academic Press, New York.

Cox, D.T. \& Shin, S. 2003 Laboratory measurements of void fraction and turbulence in the bore region of surf zone waves. J. Eng. Mech. 129, 1197-1205.

Deane, G. B. \& Stokes, M. D. 2002 Scale dependence of bubble creation mechanisms in breaking waves. Nature 418, 839-844.

Drazen, D. A. \& Melville, W. K. 2009 Turbulence and mixing in unsteady breaking surface waves. J. Fluid Mech. 628, 85.

Drazen, D. A., Melville, W. K. \& Lenain, L. 2008 Inertial scaling of dissipation in unsteady breaking waves. J. Fluid Mech. 611, 307-332.

Drew, D. 1983 Mathematical modeling of two-phase flow. Ann. Rev. Fluid Mech. 15, 261-291. 
Duncan, J. H. 1981 An experimental investigation of breaking waves produced by a towed hydrofoil. Proc. R. Soc. of Lond. A 377, 331-348.

DunCAN, J. H. 1983 The breaking and non-breaking wave resistance of a twodimensional hydrofoil. J. Fluid Mech. 126, 507-520.

Duncan, J. H. 2001 Spilling breakers. Ann. Rev. Fluid Mech. 33, 519-547.

Fox, R.O. 2012 Large-eddy-simulation tools for multiphase flows. Ann. Rev. Fluid Mech. 44, 47-76.

Garrett, C., Li, M. \& FArmer, D. 2000 The connection between bubble size spectra and energy dissipation rates in the upper ocean. J. Phys. Oceanogr. 30, 2163-2171.

Germano, M., Piomelli, U., Moin, P. \& Cabot, W. H. 1991 A dynamic subgridscale eddy viscosity model. Phys. Fluids 3, 1760-1765.

IAfrATi, A. 2009 Numerical study of the effects of the breaking intensity on wave breaking flows. J. Fluid Mech. 622, 371-411.

IAfRATI, A. 2011 Energy dissipation mechanisms in wave breaking processes: Spilling and highly aerated plunging breaking events. J. Geophys. Res 116, C07024, doi:10.1029/2011JC007038.

Kiger, K. T. \& Duncan, J. H. 2012 Air-entrainment mechanisms in plunging jets and breaking waves. Ann. Rev. Fluid Mech. 44, 563-596.

Kirby, J. T., Ma, G., Derakhti, M. \& Shi, F. 2012 Numerical investigation of turbulent bubbly flow under breaking waves. In Proc. 33d Int. Conf. Coastal Eng., pp. waves-66. Santander.

Lakehal, D. \& Liovic, P. 2011 Turbulence structure and interaction with steep breaking waves. J. Fluid Mech. 674, 522-577.

Lakehal, D., Smith, B. L. \& Milelli, M. 2002 Large-eddy simulation of bubbly turbulent shear flows. J. Turbulence 3, N25.

Lamarre, E. \& Melville, W. K. 1991 Air entrainment and dissipation in breaking waves. Nature 351, 469-472.

Lamarre, E. \& Melville, W. K. 1994 Void-fraction measurements and soundspeed fields in bubble plumes generated by breaking waves. J. Acoust. Soc. Am. 95, $1317-1328$.

Lance, M. \& Bataille, J. 1991 Turbulence in the liquid phase of a uniform bubbly air-water flow. J. Fluid Mech. 222, 95-118. 
Lilly, D. K. 1992 A proposed modification of the germano subgrid-scale closure method. Phys. Fluids 4, 633-635.

Lin, M.-Y., Moeng, C.-H., Tsai, W.-T., Sullivan, P. P. \& Belcher, S. E. 2008 Direct numerical simulation of wind-wave generation processes. J. Fluid Mech. 616, 1-30.

Lubin, P. \& Glockner, S. 2013 Numerical simulations of three-dimensional plunging breaking waves: generation and evolution of aerated vortex filaments. J. Fluid Mech. Submitted. .

Lubin, P., Vincent, S., Abadie, S. \& Caltagirone, J. 2006 Three-dimensional large eddy simulation of air entrainment under plunging breaking waves. Coastal Eng. 53 (8), 631-655.

MA, G. 2012 Multiscale numerical study of turbulent flow and bubble entrainment in the surf zone. PhD thesis, University of Delaware, Newark DE.

MA, G., Shi, F. \& Kirby, J. T. 2011 A polydisperse two-fluid model for surf zone bubble simulation. J. Geophys. Res. 116, C05010, doi:10.1029/2010JC006667.

Magnaudet, J. \& EAmes, I. 2000 The motion of high-reynolds-number bubbles in inhomogeneous flows. Ann. Rev. Fluid Mech. 32, 659-708.

Martínez-BazÁn, C., Montañés, J. L. \& Lasheras, J. C. $1999 a$ On the breakup of an air bubble injected into a fully developed turbulent flow. part 1. breakup frequency. J. Fluid Mech 401, 157-182.

Martínez-BazÁn, C., Montañés, J. L. \& Lasheras, J. C. 1999 b On the breakup of an air bubble injected into a fully developed turbulent flow. part 2. size pdf of the resulting daughter bubbles. J. Fluid Mech. 401, 183-207.

Martínez-Bazán, C., Rodríguez-Rodríguez, J., Deane, G. B., Montañés, J. L. M. \& LASheras, J. C. 2010 Considerations on bubble fragmentation models. J. Fluid Mech 661, 159-177.

Maxey, M. R. \& Riley, J. J. 1983 Equation of motion for a small rigid sphere in a nonuniform flow. Phys. Fluids 26, 883.

Melville, W. K. 1994 Energy dissipation by breaking waves. J. Phys. Oceanogr. 24, 2041-2049.

Melville, W. K. 1996 The role of surface-wave breaking in air-sea interaction. Ann. Rev. Fluid Mech. 28, 279-321.

Melville, W. K., Veron, F. \& White, C. J. 2002 The velocity field under breaking waves: coherent structures and turbulence. J. Fluid Mech. 454, 203-234. 
Moraga, F. J., Carrica, P. M., Drew, D. A. \& Lahey Jr, R. T. 2008 A subgrid air entrainment model for breaking bow waves and naval surface ships. Comp. Fluids 37, 281-298.

Perlin, M., Choi, W. \& Tian, Zh. 2012 Breaking waves in deep and intermediate waters. Ann. Rev. Fluid Mech. 45, 115-145.

Phillips, O. M. 1985 Spectral and statistical properties of the equilibrium range in wind-generated gravity waves. J. Fluid Mech. 156, 505-31.

Pope, S. B. 2000 Turbulent flows. Cambridge University Press.

Rapp, R. J. \& Melville, W. K. 1990 Laboratory measurements of deep-water breaking waves. Phil. Trans. Roy. Soc. A, 331, 735-800.

Rider, W. J. \& Kothe, D. B. 1998 Reconstructing volume tracking. J. Comp. Phys. 141, 112-152.

Rojas, G. \& Loewen, M. R. 2010 Void fraction measurements beneath plunging and spilling breaking waves. J. Geophys. Res. 115, C08001,doi:10.1029/2009JC005614.

Saruwatari, A., Watanabe, Y. \& Ingram, D. M. 2009 Scarifying and fingering surfaces of plunging jets. Coastal Eng. 56, 1109-1122.

Sato, Y. \& Sekoguchi, K. 1975 Liquid velocity distribution in two-phase bubble flow. Int. J. Multiphase Flow 2, 79-95.

Shen, L. \& Yue, D. K. P. 2001 Large-eddy simulation of free-surface turbulence. J. Fluid Mech. 440, 75-116.

Shi, F., Kirby, J. T. \& MA, G. 2010 Modeling quiescent phase transport of air bubbles induced by breaking waves. Ocean Modelling 35, 105-117.

Smagorinsky, J. 1963 General circulation experiments with the primitive equations. Monthly Weather Review 91, 99-164.

Song, Ch. \& Sirviente, A. 2004 A numerical study of breaking waves. Phys. Fluids 16, 2649-2667.

Svendsen, I. A. 1987 Analysis of surf zone turbulence. J. Geophys. Res. 92 (C5), $5115-5124$.

Van Milligen, B Ph, Hidalgo, C \& Sanchez, E 1995 Nonlinear phenomena and intermittency in plasma turbulence. Physical review letters 74, 395.

Vremen, B., Geurts, B. \& Kuerten, H. 1997 Large-eddy simulation of the turbulent mixing layer. J. Fluid Mech. 339, 357-390. 
Watanabe, Y., Saeki, H. \& Hosking, R. J. 2005 Three-dimensional vortex structures under breaking waves. J. Fluid Mech. 545, 291-328.

Zang, Y., Street, R. L. \& Koseff, J. R. 1993 A dynamic mixed subgrid-scale model and its application to turbulent recirculating flows. Phys. Fluids A 5, 31863196. 


\section{Appendix A VOID VS ACTUAL AIR}

To study breaking waves in laboratory scale with 3D LES VOF-based models, the common practice is to neglect the computations in the air side by replacing the air by void (Christensen \& Deigaard 2001, Watanabe et al. 2005, Christensen 2006). An exception is Lakehal \& Liovic (2011) where actual air density and viscosity was considered. In all the studies the mesh resolution in the stream wise direction is much coarser than the other two directions, $\frac{\Delta x}{\Delta y}=\frac{\Delta x}{\Delta z} \sim 5$ (as compared to $\sim 3$ in the present study). The effects of replacing the air by void and coarse resolution in one direction in the entrainment process especially the cavity formation and break-up process should be addressed. Figure A.1 shows that if we have enough spatial resolution the initial cavity shape and volume was captured fairly accurate compared to measurements even by $2 \mathrm{~d}$ grid with void/water interface. To examine the effects on the break up process, we design a simple bubble ring formation test by putting a cavity, $0.3 \mathrm{~m}$ below the free surface in quiescent water. Figure A.2 shows the results of cavity evolution for the air/water and void/water cases where the mesh resolution is $23.0 \times 7.0 \times 7.0 \mathrm{~mm}$; and also the results for the air/water case with the mesh resolution of $7.0 \times 7.0 \times 7.0 \mathrm{~mm}$. It is clear that with the coarser resolution the true break up can not capture even with modeling actual air, but at the coarser resolution the results with air or void are comparable. Figure A.3 shows the centroid position of cavity for the three cases. As we can see the rising velocity in the case with void are comparable to the model with air and it is because the pressure difference or buoyancy force is considered in all of the cases. We should note that the volume of cavity does not conserve in the case with void because of the semi-compressible treatment in the cells with $0<\alpha^{l}<1$. We should mention that we only ignore the computations in the cells that are filled 

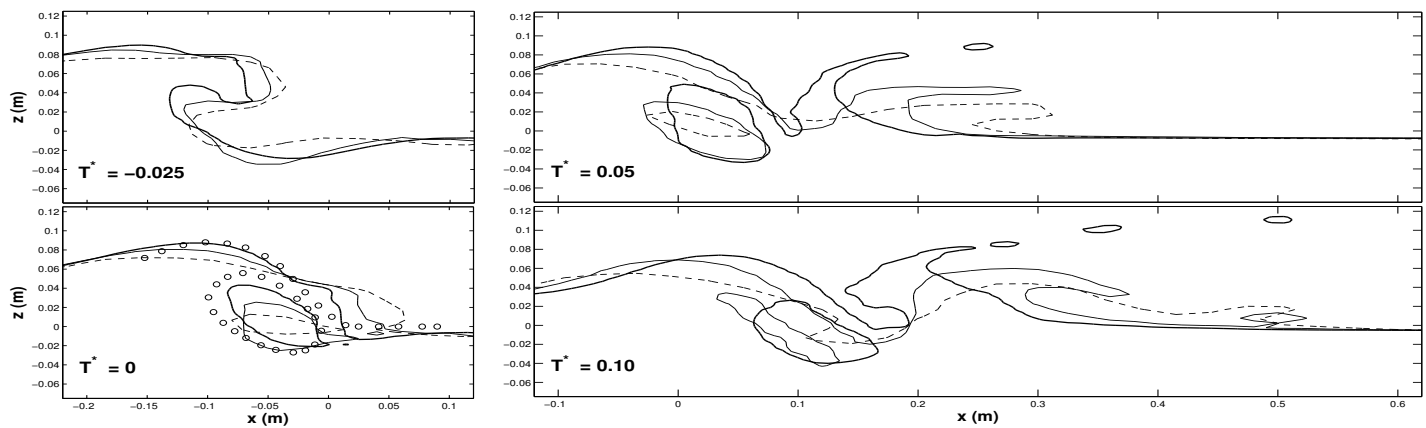

Figure A.1: Snapshots of the time sequences of the overturning process and splash-up for P5. Free surface profiles (contours of $\alpha^{l}=0.5$ ) are shown for different mesh resolutions $(\Delta x \times \Delta z),-10 \times 2.5 \mathrm{~mm},-20 \times 5 \mathrm{~mm},---30 \times$ $10 \mathrm{~mm}$. Circles shows the measurement (adopted from BCh figure 3).

completely by void $\alpha^{l}=0$ (e.g. above the free surface) and then if we increase the resolution in the void/water model there are a lot of cells, filled only by void and then they can freely squeeze and we lose the main volume of the cavity. We can conclude that the void/water model can capture the free surface evolution before breaking, the cavity shape and volume. The overall cavity evolution and integrated rising velocity can be captured for the void pocket with the dimension of $<2 \Delta x$. 

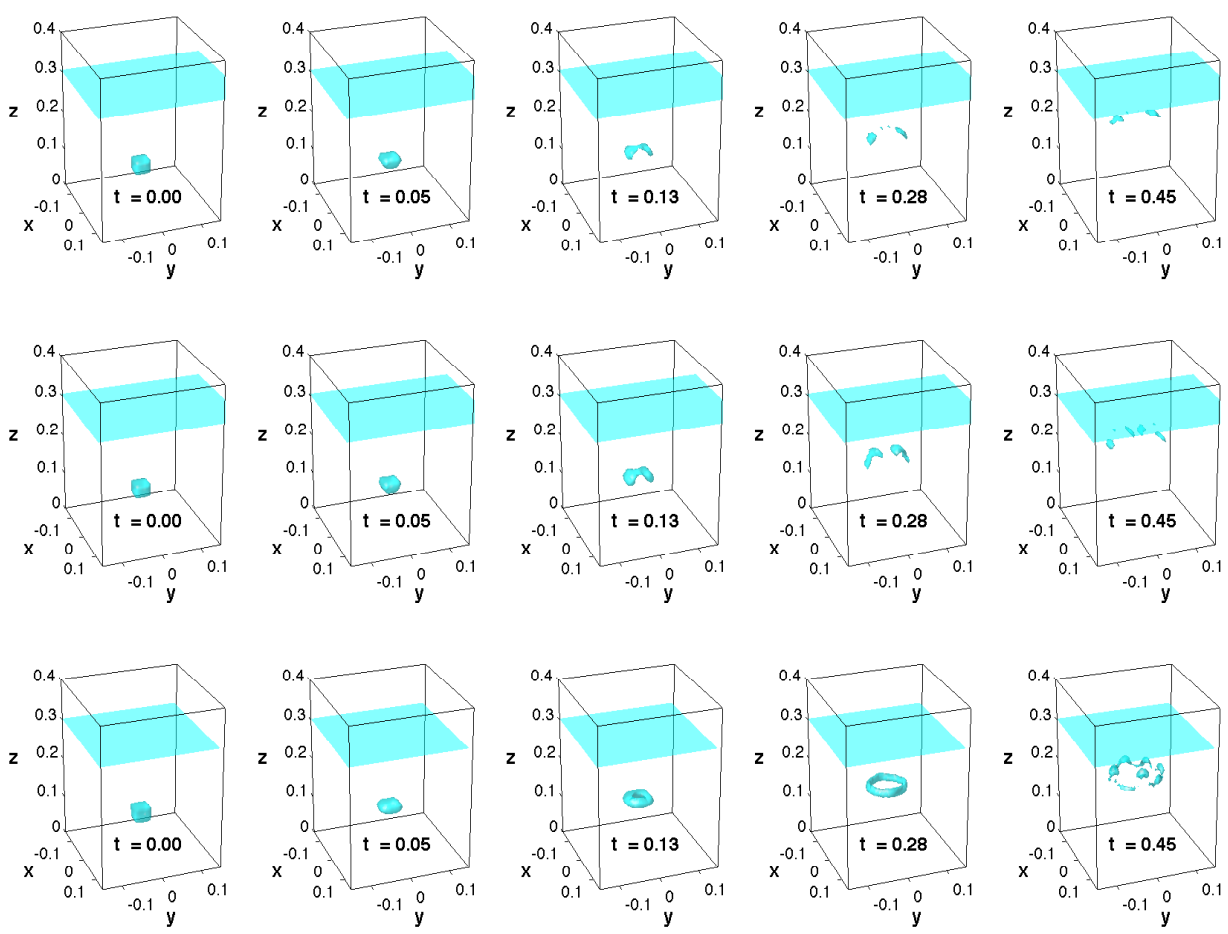

Figure A.2: Cavity deformation in the quiescent water (Iso-surface of $\alpha=0.5$ ); (first row) water/void with $23 \times 7 \times 7 \mathrm{~mm}$ resolution; (2nd row) water/air with $23 \times 7 \times 7 \mathrm{~mm}$ resolution; (3rd row) water/air with $7 \times 7 \times 7 \mathrm{~mm}$ resolution

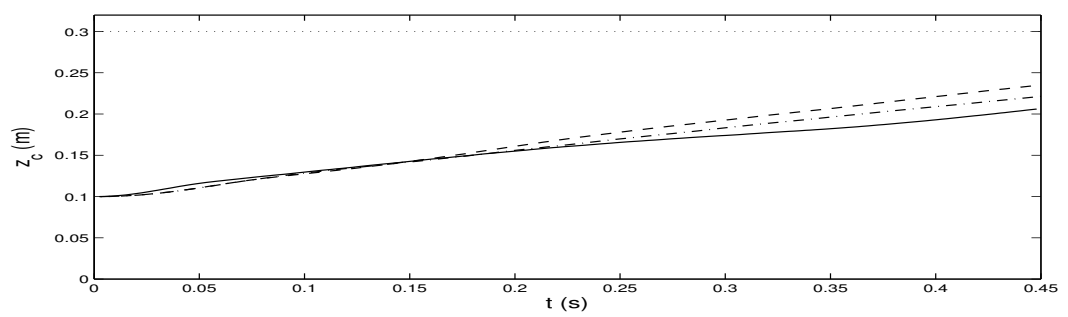

Figure A.3: Centroid position of cavity; - - - water/void with $23 \times 7 \times 7 \mathrm{~mm}$ resolution; - - - water/air with $23 \times 7 \times 7 \mathrm{~mm}$ resolution; _ water/air with $7 \times$ $7 \times 7 \mathrm{~mm}$ resolution; $\cdots \cdots$ shows the free surface location 


\section{Appendix B}

\section{EXACT TRASPORT EQUATIONS FOR TURBULENT BUBBLY FLOW}

The Reynolds decomposition of the resolved filed $\tilde{\phi}=<\tilde{\phi}>+\tilde{\phi}^{\prime}$ is used to separate organized and turbulent motions, where $<>$ could be ensemble or phase averaging and here is approximated by spanwise averaging given by (2.35). All the variables are the liquid phase resolved quantities and for simplicity we drop $\left(\tilde{)}\right.$ and ()$^{l}$.

\section{B.1 Transport Equation For Averaged Resolved Kinetic Energy}

Resolved kinetic energy per unit mass $\left(e_{k}=\frac{1}{2} u_{i} u_{i}\right)$ equation can be obtained from multiplying the liquid phase momentum equation, (2.14), by $u_{i}$

$$
u_{i} \times[\underbrace{\frac{\partial\left(\rho \alpha u_{i}\right)}{\partial t}}_{\mathrm{a}}+\underbrace{\frac{\partial\left(\rho \alpha u_{i} u_{j}\right)}{\partial x_{j}}}_{\mathrm{b}}=\underbrace{-\frac{\partial(\alpha P)}{\partial x_{j}} \delta_{i j}}_{\mathrm{c}}+\underbrace{\rho \alpha g_{i}}_{\mathrm{d}}+\underbrace{\frac{\partial}{\partial x_{j}}\left[\rho \alpha\left(2 \nu \mathcal{S}_{i j}-\tau_{i j}^{d}\right)\right.}_{\mathrm{e}}] \underbrace{-f_{i}}_{\mathrm{f}}]
$$

where $P=p+\frac{2}{3} \rho k_{s g s}$ is the modified pressure, $-\tau_{i j}^{d}=2 \nu_{s g s} \mathcal{S}_{i j}$ is the deviatoric part of the SGS stress tensor and $f_{i}$ is the hydrodynamic forces exerted on the dispersed bubbles. By using the chain rule and $A \frac{\partial A B}{\partial x}=\frac{\partial C B}{\partial x}+C \frac{\partial B}{\partial x}$ where $C=\frac{1}{2} A^{2}$, we obtain

$$
\begin{aligned}
u_{i} \times a & : \frac{\partial \rho \alpha e_{k}}{\partial t}+e_{k} \frac{\partial \rho \alpha}{\partial t} \\
u_{i} \times b & : \frac{\partial \rho \alpha u_{j} e_{k}}{\partial x_{j}}+e_{k} \frac{\partial \rho \alpha u_{j}}{\partial x_{j}} \\
u_{i} \times c \quad: & -\frac{\partial \alpha P u_{j}}{\partial x_{j}}+\alpha P \frac{\partial u_{j}}{\partial x_{j}} \\
u_{i} \times d & : \quad \rho \alpha g_{i} u_{i} \\
u_{i} \times e & : \quad \frac{\partial}{\partial x_{j}}\left[\rho \alpha\left(2 \nu \mathcal{S}_{i j}-\tau_{i j}^{d}\right) u_{i}\right]-\rho \alpha\left(2 \nu \mathcal{S}_{i j} \mathcal{S}_{i j}-\tau_{i j}^{d} \mathcal{S}_{i j}\right) \\
u_{i} \times f & : \quad-f_{i} u_{i} .
\end{aligned}
$$


Using continuity equation (2.13) the sumation of $e_{k} \frac{\partial}{\partial}$ terms are equal to zero, and the transport equation of averaged resolved kinetic energy is obtained by averaging the remaining terms of (B.2) as

$$
\frac{\partial}{\partial t}\left(\rho \overline{\alpha e_{k}}\right)+\frac{\partial}{\partial x_{j}}\left(\rho \overline{\alpha u_{j} e_{k}}\right)=T-\left(\epsilon_{r}+\epsilon_{s g s}\right)+D+B+E
$$

where

$$
\begin{aligned}
T & =-\frac{\partial}{\partial x_{j}}\left[\overline{\alpha P u_{j}}-\rho \overline{\alpha\left(2 \nu \mathcal{S}_{i j}-\tau_{i j}^{d}\right) u_{i}}\right] \text { (rate of work done by pressure and viscous stresses) } \\
\epsilon_{r} & =2 \rho \overline{\alpha \nu \mathcal{S}_{i j} \mathcal{S}_{i j}} \quad \text { (viscous dissipation rate) } \\
\epsilon_{s g s} & =-\frac{\rho \alpha \tau_{i j}^{d} \mathcal{S}_{i j}}{\alpha} \quad \text { (SGS dissipation rate) } \\
D & =\alpha \frac{\partial u_{j}}{\partial x_{j}} \quad \text { (pressure dilatation rate) } \\
B & =-\overline{f_{i} u_{i}} \quad(\text { rate of work done by dispersed bubbles) } \\
E & =\rho \overline{\alpha g_{i} u_{i}}=\frac{D}{D t}\left(-\rho \overline{\alpha e_{p}}\right) \quad \text { (rate of the total change of the potential energy) }
\end{aligned}
$$

where $e_{p}=g z$ is the potential energy per unit mass and $g=\left|g_{3}\right|$.

\section{B.2 Transport Equation For Resolved Turbulent Kinetic Energy}

The transport equation for averaged resolved turbulent kinetic energy per unit mass, $\bar{k}=\frac{1}{2} \overline{u_{i}^{\prime} u_{i}^{\prime}}$, is obtained from multiplying the liquid phase momentum equation by $u_{i}^{\prime}$ and then perform averaging.

$$
u_{i}^{\prime} \times[\underbrace{\frac{\partial\left(\rho \alpha u_{i}\right)}{\partial t}}_{\mathrm{a}}+\underbrace{\frac{\partial\left(\rho \alpha u_{i} u_{j}\right)}{\partial x_{j}}}_{\mathrm{b}}=\underbrace{-\frac{\partial(\alpha P)}{\partial x_{j}} \delta_{i j}}_{\mathrm{c}}+\underbrace{\rho \alpha g_{i}}_{\mathrm{d}}+\underbrace{\frac{\partial}{\partial x_{j}}\left[\rho \alpha\left(2 \nu \mathcal{S}_{i j}-\tau_{i j}^{d}\right)\right.}_{\mathrm{e}} \underbrace{-f_{i}}_{\mathrm{f}}]
$$


Note that only fluctuating part of each term will survive after averaging. By using the chain rule and $A \frac{\partial A B}{\partial x}=\frac{\partial C B}{\partial x}+C \frac{\partial B}{\partial x}$ where $C=\frac{1}{2} A^{2}$, we can rewrite (B.5) as

$$
\begin{aligned}
& \overline{u_{i}^{\prime} \times a}: \overline{u_{i}^{\prime} \frac{\partial}{\partial t} \rho\left(\bar{\alpha} u_{i}^{\prime}+\alpha^{\prime} \bar{u}_{i}+\alpha^{\prime} u_{i}^{\prime}\right)}=\overline{\frac{\partial \rho \bar{\alpha} k}{\partial t}}+\overline{k \frac{\partial \rho \bar{\alpha}}{\partial t}}+\overline{\alpha^{\prime} u_{i}^{\prime}} \frac{\partial \rho \bar{u}_{i}}{\partial t}+\overline{\overline{\frac{\partial \rho \alpha^{\prime}}{\partial t}}}+\overline{\frac{\partial \rho \alpha^{\prime} k}{\partial t}}+\overline{k \frac{\partial \rho \alpha^{\prime}}{\partial t}} \\
& \left.\overline{u_{i}^{\prime} \times b}: \overline{u_{i}^{\prime} \frac{\partial}{\partial x_{j}}\left[\rho\left(u_{i}^{\prime} \bar{\alpha} \bar{u}_{j}+\bar{\alpha} \bar{u}_{i} u_{j}^{\prime}+\bar{\alpha} u_{i}^{\prime} u_{j}^{\prime}+\alpha^{\prime} \bar{u}_{i} \bar{u}_{j}+\bar{u}_{i} \alpha^{\prime} u_{j}^{\prime}+\bar{u}_{j} \alpha^{\prime} u_{i}^{\prime}+\alpha^{\prime} u_{i}^{\prime} u_{j}^{\prime}\right)\right.}\right]= \\
& \overline{\frac{\partial \rho \bar{\alpha} \bar{u}_{j} k}{\partial x_{j}}}+\overline{k \frac{\partial \rho \bar{\alpha} \bar{u}_{j}}{\partial x_{j}}}+\overline{\frac{\partial \rho \bar{\alpha} u_{j}^{\prime}}{\partial x_{j}}}+\overline{\bar{\alpha} u_{i}^{\prime} u_{j}^{\prime} \frac{\partial \rho \bar{u}_{i}}{\partial x_{j}}}+\overline{\overline{\partial \rho \bar{\alpha} u_{j}^{\prime} k}} \frac{\overline{\partial \rho \bar{\alpha} u_{j}^{\prime}}}{\partial x_{j}}+\overline{\frac{\partial \rho \alpha^{\prime} \bar{u}_{j}}{\partial x_{j}}} \\
& +\overline{\bar{u}_{j} \alpha^{\prime} u_{i}^{\prime} \frac{\partial \rho \bar{u}_{i}}{\partial x_{j}}}+\overline{\frac{\partial \rho \alpha^{\prime} u_{j}^{\prime}}{\partial x_{j}}}+\overline{\alpha^{\prime} u_{i}^{\prime} u_{j}^{\prime} \frac{\partial \rho \bar{u}_{i}}{\partial x_{j}}}+\overline{\frac{\partial \rho \alpha^{\prime} \bar{u}_{j} k}{\partial x_{j}}}+\overline{\overline{\frac{\partial \rho \alpha^{\prime} \bar{u}_{j}}{\partial x_{j}}}}+\frac{\overline{\partial \rho \alpha^{\prime} u_{j}^{\prime} k}}{\partial x_{j}}+\overline{\frac{\partial \rho \alpha^{\prime} u_{j}^{\prime}}{\partial x_{j}}} \\
& \overline{u_{i}^{\prime} \times c}:-\overline{\frac{\partial \bar{\alpha} P^{\prime} u_{j}^{\prime}}{\partial x_{j}}}+\overline{\bar{\alpha} P^{\prime} \frac{\partial u_{j}^{\prime}}{\partial x_{j}}}-\overline{\frac{\partial \alpha^{\prime} P^{\prime} u_{j}^{\prime}}{\partial x_{j}}}+\overline{\alpha^{\prime} P^{\prime} \frac{\partial u_{j}^{\prime}}{\partial x_{j}}}-\overline{\frac{\partial \bar{P} \alpha^{\prime} u_{j}^{\prime}}{\partial x_{j}}}+\overline{\bar{P} \alpha^{\prime} \frac{\partial u_{j}^{\prime}}{\partial x_{j}}} \\
& \overline{u_{i}^{\prime} \times d}: \rho g_{i} \overline{\alpha^{\prime} u_{i}^{\prime}} \\
& \overline{u_{i}^{\prime} \times e} \quad: \quad \overline{\frac{\partial}{\partial x_{j}}\left[\rho \bar{\alpha}\left(2 \nu \mathcal{S}^{\prime}{ }_{i j}-\tau_{i j}^{\prime d}\right) u_{i}^{\prime}\right]}-\rho \bar{\alpha}\left(\overline{2 \nu \mathcal{S}^{\prime}{ }_{i j} \mathcal{S}^{\prime}{ }_{i j}}-\overline{\tau_{i j}^{\prime d} \mathcal{S}^{\prime}{ }_{i j}}\right)
\end{aligned}
$$

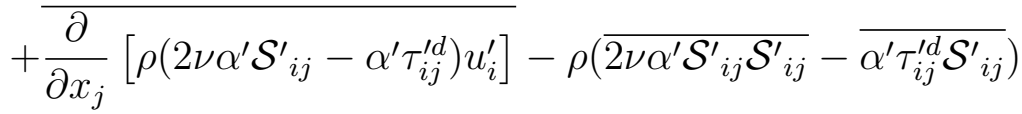

$$
\begin{aligned}
& +\overline{\frac{\partial}{\partial x_{j}}\left[\rho\left(2 \nu \alpha^{\prime} u_{i}^{\prime} \overline{\mathcal{S}}_{i j}-\alpha^{\prime} u_{i}^{\prime} \bar{\tau}_{i j}^{d}\right)\right]}-\rho\left(\overline{2 \nu \alpha^{\prime} \mathcal{S}^{\prime}{ }_{i j} \overline{\mathcal{S}}_{i j}}-\overline{\alpha^{\prime} \mathcal{S}^{\prime}{ }_{i j} \bar{\tau}_{i j}^{d}}\right) \\
& \overline{u_{i}^{\prime} \times f}:-\overline{f_{i}^{\prime} u_{i}^{\prime}} \text {, }
\end{aligned}
$$

where $r=u_{i}^{\prime} \bar{u}_{i}$. Using continuity equation, the summation of $\overline{k \frac{\partial}{\partial}}$ and $\overline{r \frac{\partial}{\partial}}$ terms are equal to zero, thus (B.6) simplified to

$$
\begin{aligned}
\frac{\partial}{\partial t}(\rho \bar{\alpha} \bar{k})+\frac{\partial}{\partial x_{j}}\left(\rho \bar{\alpha} \bar{u}_{j} \bar{k}\right) & =T^{k}+P^{k}+B^{k}+E^{k}-\left(\epsilon_{r}^{k}+\epsilon_{s g s}^{k}\right)+D^{k} \\
& +t_{e x}^{k}+T_{e x}^{k}+P_{e x}^{k}-\left(\epsilon_{r, e x}^{k}+\epsilon_{s g s, e x}^{k}\right)+D_{e x}^{k},
\end{aligned}
$$

where $k=\frac{1}{2} u_{i}^{\prime} u_{i}^{\prime}$ is the resolved TKE and 


$$
\begin{aligned}
T^{k}= & -\frac{\partial}{\partial x_{j}}\left[\bar{\alpha} \overline{P^{\prime} u_{j}^{\prime}}+\rho \bar{\alpha} \overline{u_{j}^{\prime} k}-\rho \bar{\alpha} \overline{\left(2 \nu \mathcal{S}^{\prime}{ }_{i j}-\tau_{i j}^{\prime d}\right) u_{i}^{\prime}}\right] \quad \text { (press } \\
& \text { and SGS trasport rate) } \\
P^{k}= & -\rho \bar{\alpha} \overline{u_{i}^{\prime} u_{j}^{\prime}} \frac{\partial \bar{u}_{i}}{\partial x_{j}}, \quad \text { (production rate by mean shear) } \\
B^{k}= & -\overline{f_{i}^{\prime} u_{i}^{\prime}}, \quad \text { (production rate by the dispersed bubbles) } \\
E^{k}= & -\rho g \overline{\alpha^{\prime} u_{3}^{\prime}}, \quad \text { (production rate by buoyancy) } \\
\epsilon_{r}^{k}= & 2 \rho \nu \bar{\alpha} \overline{\mathcal{S}^{\prime}{ }_{i j} \mathcal{S}^{\prime}}{ }_{i j}, \quad \text { (viscous dissipation rate) } \\
\epsilon_{s g s}^{k}= & -\rho \bar{\alpha} \overline{\tau_{i j}^{\prime d} \mathcal{S}^{\prime}{ }_{i j}}, \quad \text { (SGS dissipation rate) } \\
D^{k}= & \bar{\alpha} P^{\prime} \frac{\partial u_{j}^{\prime}}{\partial x_{j}}, \quad \text { (pressure dilatation rate) }
\end{aligned}
$$

(pressure, turbulent, viscous,

and the extra terms due to the correlation of $\alpha^{\prime}$ with velocity and pressure fluctuations,

$$
\begin{aligned}
T_{e x}^{k}= & -\frac{\partial}{\partial x_{j}}\left[\overline{\alpha^{\prime} P^{\prime} u_{j}^{\prime}}+\bar{P} \overline{\alpha^{\prime} u_{j}^{\prime}}+\rho \overline{\alpha^{\prime} k} \bar{u}_{j}+\rho \overline{\alpha^{\prime} u_{j}^{\prime} k}-\rho \overline{\left(2 \nu \alpha^{\prime} \mathcal{S}^{\prime}{ }_{i j}-\alpha^{\prime} \tau_{i j}^{\prime d}\right) u_{i}^{\prime}}\right. \\
& \left.-\rho\left(2 \nu \overline{\alpha^{\prime} u_{i}^{\prime}} \overline{\mathcal{S}}_{i j}-\overline{\alpha^{\prime} u_{i}^{\prime}} \bar{\tau}_{i j}^{d}\right)\right], \quad(\text { extra transport rate terms) } \\
t_{e x}^{k}= & -\frac{\overline{\partial \rho \alpha^{\prime} k}}{\partial t}-\overline{\alpha^{\prime} u_{i}^{\prime}} \frac{\partial \rho \bar{u}_{i}}{\partial t}, \quad(\text { extra transient terms }) \\
P_{e x 1}^{k}= & -\rho \bar{u}_{j} \overline{\alpha^{\prime} u_{i}^{\prime}} \frac{\partial \bar{u}_{i}}{\partial x_{j}}, \quad P_{e x 2}^{k}=-\rho \overline{\alpha^{\prime} u_{i}^{\prime} u_{j}^{\prime}} \frac{\partial \bar{u}_{i}}{\partial x_{j}}, \quad \text { (extra production terms) } \\
\epsilon_{r, e x 1}^{k}= & 2 \rho \nu \overline{\alpha^{\prime} \mathcal{S}^{\prime}{ }_{i j} \mathcal{S}^{\prime}{ }_{i j}}, \quad \epsilon_{r, e x 2}^{k}=2 \rho \nu \overline{\alpha^{\prime} \mathcal{S}^{\prime}{ }_{i j}} \overline{\mathcal{S}}_{i j}, \quad \text { (extra viscous dissipation terms) } \\
\epsilon_{s g s, e x 1}^{k}= & -\rho \overline{\alpha^{\prime} \tau_{i j}^{\prime d} \mathcal{S}^{\prime}{ }_{i j}}, \quad \frac{\epsilon_{s g s, e x 2}^{k}}{k}=-\rho \overline{\alpha^{\prime} \mathcal{S}^{\prime}{ }_{i j}} \bar{\tau}_{i j}^{d}, \quad \text { (extra SGS dissipation terms) } \\
D_{e x}^{k}= & \alpha^{\prime} P^{\prime} \frac{\partial u_{j}^{\prime}}{\partial x_{j}}+\bar{P} \alpha^{\prime} \frac{\partial u_{j}^{\prime}}{\partial x_{j}}, \quad \text { (extra pressure dilatation terms). }
\end{aligned}
$$

Note that the production terms are identical in the transport equation of organized flow kinetic energy and TKE. In addition

$$
\epsilon_{r}=\epsilon_{r}^{m}+\epsilon_{r, e x}^{m}+\epsilon_{r}^{k}+\epsilon_{r, e x}^{k}, \quad \epsilon_{s g s}=\epsilon_{s g s}^{m}+\epsilon_{s g s, e x}^{m}+\epsilon_{s g s}^{k}+\epsilon_{s g s, e x}^{k} .
$$


In the case of a single phase flow $\bar{\alpha}=1$ and $\alpha^{\prime}=\frac{\partial \bar{u}_{j}}{\partial x_{j}}=\frac{\partial u_{j}^{\prime}}{\partial x_{j}}=0$, by which the extra terms becomes zero and (B.12) and (B.7) reduces to the classical transport equation of organized flow kinetic energy and TKE, respectively.

\section{B.3 Transport Equation For Kinetic Energy Of Organized Flow}

The transport equation for kinetic energy of organized flow per unit mass $\left(e_{m}=\right.$ $\frac{1}{2} \overline{u_{i}} \overline{u_{i}}$ ) is obtained from multiplying the averaged liquid phase momentum equation by $\overline{u_{i}}$

$$
\overline{u_{i}} \times[\underbrace{\frac{\partial\left(\overline{\rho \alpha u_{i}}\right)}{\partial t}}_{\mathrm{a}}+\underbrace{\frac{\partial\left(\overline{\rho \alpha u_{i} u_{j}}\right)}{\partial x_{j}}}_{\mathrm{b}}=\underbrace{-\frac{\partial(\overline{\alpha P})}{\partial x_{j}} \delta_{i j}}_{\mathrm{c}}+\underbrace{\overline{\rho \alpha g_{i}}}_{\mathrm{d}}+\underbrace{\frac{\partial}{\partial x_{j}}\left[\overline{\rho \alpha\left(2 \nu \mathcal{S}_{i j}-\tau_{i j}^{d}\right)}\right]}_{\mathrm{e}} \underbrace{-\overline{f_{i}}}_{\mathrm{f}}]
$$

With taking the procedure explained in the previous section and using the averaged continuity equation, the transport equation for kinetic energy of organized flow given by

$$
\begin{aligned}
\frac{\partial}{\partial t}\left(\rho \bar{\alpha} e_{m}\right)+\frac{\partial}{\partial x_{j}}\left(\rho \bar{\alpha} \bar{u}_{j} e_{m}\right) & =T^{m}-P^{m}-\left(\epsilon_{r}^{m}+\epsilon_{s g s}^{m}\right)+D^{m}+B^{m}+E^{m} \\
& +t_{e x}^{m}+T_{e x}^{m}-P_{e x}^{m}-\left(\epsilon_{r, e x}^{m}+\epsilon_{s g s, e x}^{m}\right)+D_{e x}^{m}
\end{aligned}
$$

where

$$
\begin{aligned}
T^{m} & =-\frac{\partial}{\partial x_{j}}\left(\bar{\alpha} \bar{P} \bar{u}_{j}-\rho \bar{\alpha}\left(2 \nu \overline{\mathcal{S}}_{i j}+\bar{\tau}_{i j}^{d}\right) \bar{u}_{i}+\rho \bar{\alpha} \bar{u}_{i} \overline{u_{i}^{\prime} u_{j}^{\prime}}\right), \quad P^{m}=-\rho \bar{\alpha} \overline{u_{i}^{\prime} u_{j}^{\prime}} \frac{\partial \bar{u}_{i}}{\partial x_{j}} \\
\epsilon_{r}^{m} & =2 \rho \nu \bar{\alpha} \overline{\mathcal{S}}_{i j} \overline{\mathcal{S}}_{i j}, \quad \epsilon_{s g s}^{m}=-\rho \bar{\alpha} \bar{\tau}_{i j}^{d} \overline{\mathcal{S}}_{i j}, \quad D^{m}=\bar{\alpha} \bar{P} \frac{\partial \bar{u}_{j}}{\partial x_{j}}, \\
B^{m} & =-\bar{f}_{i} \bar{u}_{i}, \quad E^{m}=\rho g_{i} \bar{\alpha} \bar{u}_{i}=\frac{D}{D t}\left(-\rho \bar{\alpha} \bar{e}_{p}\right),
\end{aligned}
$$


and the extra terms due to $\alpha^{\prime}$ correlation with velocity and pressure fluctuations are

$$
\begin{aligned}
T_{e x}^{m} & =-\frac{\partial}{\partial x_{j}}\left(\overline{\alpha^{\prime} P^{\prime}} \bar{u}_{j}-\rho\left(2 \nu \overline{\alpha^{\prime} \mathcal{S}^{\prime}{ }_{i j}}+\overline{\alpha^{\prime} \tau_{i j}^{\prime d}}\right) \bar{u}_{i}+\rho \overline{\alpha^{\prime} u_{i}^{\prime} u_{j}^{\prime}} \bar{u}_{i}+\rho \overline{\alpha^{\prime} u_{j}^{\prime}} e_{m}+\rho \overline{\alpha^{\prime} u_{i}^{\prime}} \bar{u}_{i} \bar{u}_{j}\right) \\
t_{e x}^{m} & =-\overline{u_{i}} \frac{\partial\left(\rho \overline{\alpha^{\prime} u_{i}^{\prime}}\right)}{\partial t}, \quad P_{e x}^{m}=-\rho \bar{u}_{j} \overline{\alpha^{\prime} u_{i}^{\prime}} \frac{\partial \bar{u}_{i}}{\partial x_{j}}-\rho \overline{\alpha^{\prime} u_{i}^{\prime} u_{j}^{\prime}} \frac{\partial \bar{u}_{i}}{\partial x_{j}} \\
\epsilon_{r, e x}^{m} & =2 \rho \nu \overline{\alpha^{\prime} \mathcal{S}_{i j}^{\prime}} \overline{\mathcal{S}}_{i j}, \quad \epsilon_{s g s, e x}^{m}=-\rho \overline{\alpha^{\prime} \tau_{i j}^{\prime d}} \overline{\mathcal{S}}_{i j} \quad D_{e x}^{m}=\overline{\alpha^{\prime} P^{\prime}} \frac{\partial \bar{u}_{j}}{\partial x_{j}}
\end{aligned}
$$

